\title{
Sulfur and Nitrogen Modulated One-Pot Double Annulation of Arenes
}

\author{
Majji Shankar, ${ }^{\dagger}$ Arijit Saha, ${ }^{\dagger}$ Arghadip Ghosh, Somratan Sau, and Akhila K. Sahoo* \\ School of Chemistry, University of Hyderabad, Hyderabad 500046, India \\ akhilchemistry12@gmail.com / akssc@uohyd.ac.in
}

\section{SUPPORTING INFORMATION}

Table of Contents

Page

List of precursors

S2

X-ray crystal structure and data

S3-S4

References

S4

Copies of the ${ }^{1} \mathrm{H}$ NMR, and ${ }^{13} \mathrm{C}\left\{{ }^{1} \mathrm{H}\right\}$ NMR spectra of all products

S5-S114

${ }^{\dagger}$ contributed equally. 
Following the known procedure, compounds $\mathbf{1}^{\prime} \mathbf{a}, \mathbf{1}^{\prime} \mathbf{b}, \mathbf{1}^{\prime} \mathbf{c}, \mathbf{1}^{\prime} \mathbf{d}, \mathbf{1}^{\prime} \mathbf{e}, \mathbf{1}^{\prime} \mathbf{f}, \mathbf{1}^{\prime} \mathbf{g}, \mathbf{1}^{\prime} \mathbf{h}, \mathbf{1}^{\prime} \mathbf{i}, \mathbf{1}^{\prime} \mathbf{j}, \mathbf{1}^{\prime} \mathbf{k}$, 1'l, 1'm, 1'n, 1'o, 1'p, 1'q, 1'r, and 1'd-D5 were prepared. ${ }^{1}$ Symmetrical alkynes were synthesized following the reported procedures. ${ }^{2}$ Analytical and spectral data of these compounds are exactly matching with the reported values.

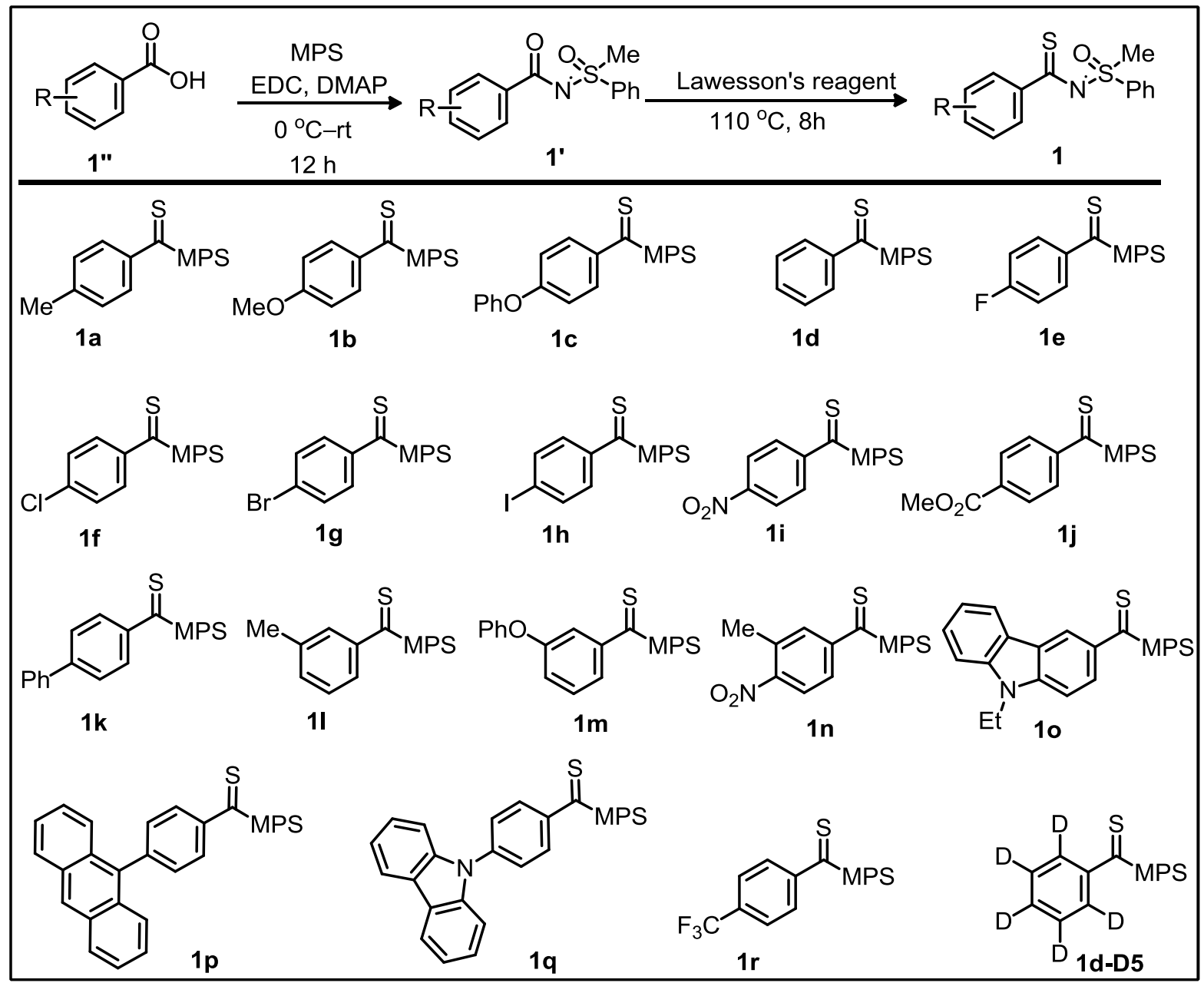

The symmetrical alkynes $2 \mathbf{e}, \mathbf{2 f}, \mathbf{2 g}, \mathbf{2 h}, \mathbf{2 i}$ and unsymmetrical alkyne (2k) were prepared. $^{2}$ 4Octyne (2a), 3-hexyne (2b), 5-decyne (2c), diphenyl acetylene (2d), 1-phenyl-1-hexyne (2j), and 2-hexyne (2l) were purchased and used.

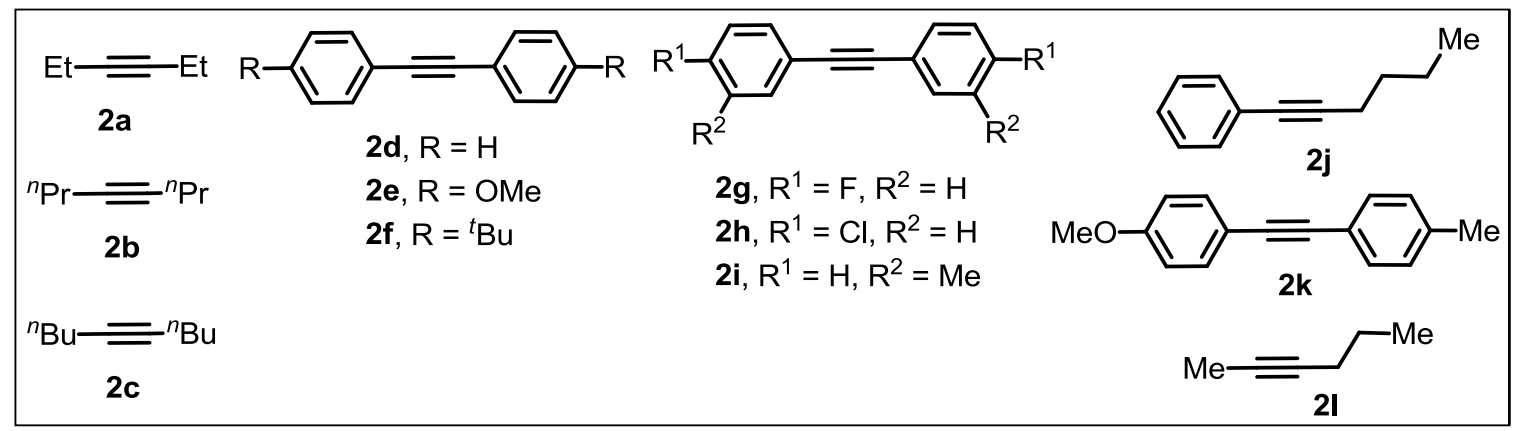


X-ray crystallography: Single crystal X-ray data for the compound 3o, 5f, and $\mathbf{5 h}$ were collected using the Bruker D8 Quest CMOS detector system $[\lambda(\mathrm{Mo}-\mathrm{K \alpha} \alpha)=0.71073 \AA]$ at 298K, graphite monochromator with a $\omega$ scan width of $0.3^{\circ}$, crystal-detector distance $60 \mathrm{~mm}$, collimator $0.5 \mathrm{~mm}$. The SMART software ${ }^{3}$ was used for the intensity data acquisition and the SAINTPLUS Software ${ }^{3}$ was used for the data extraction. In each case, absorption correction was performed with the help of SADABS program, ${ }^{3}$ an empirical absorption correction using equivalent reflections was performed with the program. The structure was solved using SHELXS-97, ${ }^{4}$ and full-matrix least-squares refinement against $\mathrm{F}^{2}$ was carried out using SHELXL-97. ${ }^{4}$ All non-hydrogen atoms were refined anisotropically. Aromatic and methyl hydrogens were introduced on calculated positions and included in the refinement riding on their respective parent atoms.

\section{X-ray crystal structure and data for 30,5 and $5 h$}

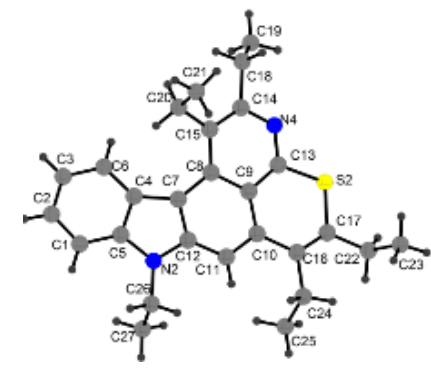

3o

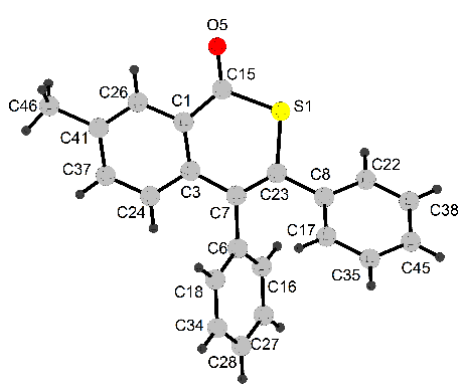

$\mathbf{5 f}$

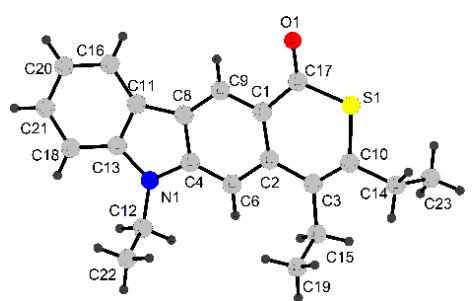

$5 \mathbf{h}$

Figure S3. Thermal ellipsoidal plot of compound 3o, $\mathbf{5 f}$, and $\mathbf{5 h}$ with atom labeling scheme. Displacement ellipsoids are drawn at 50\% probability level except for the $\mathrm{H}$ atoms, which are shown as circles of arbitrary radius. Single crystals of $\mathbf{3 0}, \mathbf{5 f}$, and $\mathbf{5 h}$ were grown by the slow evaporation of $\mathrm{CDCl}_{3}$ and DCM mixture.

Table S2. Crystal data for 3o, 5f, and $5 \mathrm{~h}$

\begin{tabular}{|c|c|c|c|}
\hline Identification code & 3o & 5f & 5h \\
\hline Formula & $\mathrm{C}_{27} \mathrm{H}_{30} \mathrm{~N}_{2} \mathrm{~S}$ & $\mathrm{C}_{22} \mathrm{H}_{16} \mathrm{OS}$ & $\mathrm{C}_{21} \mathrm{H}_{21} \mathrm{NOS}$ \\
\hline$F_{w}$ & 414.59 & 328.41 & 335.45 \\
\hline$T(\mathrm{~K})$ & 298 & 298 & 298 \\
\hline$\lambda(\AA)$ & 0.71073 & 0.71073 & 0.71073 \\
\hline Crystal system & Triclinic & Triclinic & Triclinic \\
\hline Space group & $P-1$ & $P-1$ & $P-1$ \\
\hline$a(\AA)$ & $9.1607(10)$ & $9.3550(11)$ & $8.8539(3)$ \\
\hline$b(\AA)$ & $11.5563(11)$ & $14.457(2)$ & $9.4280(4)$ \\
\hline$c(\AA)$ & $11.6613(12)$ & $18.903(3)$ & $11.2185(4)$ \\
\hline$\alpha\left({ }^{\circ}\right)$ & $103.463(4)$ & $98.904(6)$ & $86.377(2)$ \\
\hline
\end{tabular}




\begin{tabular}{|c|c|c|c|}
\hline$\beta\left(^{\circ}\right)$ & $91.605(5)$ & $91.460(5)$ & $74.258(1)$ \\
\hline$\left.\chi^{\circ}\right)$ & $109.814(4)$ & $90.490(5)$ & $73.195(1)$ \\
\hline$V\left(\AA^{3}\right)$ & $1121.8(2)$ & $2524.7(6)$ & $862.73(6)$ \\
\hline$Z$ & 2 & 6 & 2 \\
\hline$\rho_{\text {calcd }}\left(\mathrm{Mg} \mathrm{m}^{-3}\right)$ & 1.227 & 1.296 & 1.291 \\
\hline$\mu\left[\mathrm{mm}^{-1}\right]$ & 0.161 & 0.197 & 0.194 \\
\hline total reflns & 5197 & 11608 & 3524 \\
\hline unique reflns & 5162 & 11079 & 3520 \\
\hline observed reflns & 4073 & 5310 & 2596 \\
\hline$R_{1}[I>2 \sigma(I)]$ & 0.0706 & 0.0783 & 0.0490 \\
\hline$w R_{2}[$ all $]$ & 0.2112 & 0.1777 & 0.1173 \\
\hline GOF & 1.058 & 1.037 & 1.056 \\
\hline Diffractometer & SMART APEX & SMART APEX & SMART APEX \\
& CCD & CCD & CCD \\
\hline CCDC Number & 1846555 & 1917355 & 1917354 \\
\hline
\end{tabular}

\section{References:}

1. Shankar, M.; Saha, A.; Sau, S.; Ghosh, A.; Gandon, V.; Sahoo, A. K. Harnessing sulfur and nitrogen in the cobalt(III)-catalyzed unsym-metrical double annulation of thioamides: probing the origin of chemo- and regio-selectivity. Chem. Sci. 2021, 12, 6393-6405.

2. (a) M. J. Mio, L. C. Kopel, J. B. Braun, T. L. Gadzikwa, K. L. Hull, R. G. Brisbois, C. J. Markworth and P. A. Grieco, One-pot synthesis of symmetrical and unsymmetrical bisarylethynes by a modification of the Sonogashira coupling reaction. Org. Lett., 2002, 4, 3199-3202; (b) K. Park, G. Bae, J. Moon, J. Choe, K. H. Song and S. Lee, Synthesis of Symmetrical and Unsymmetrical Diarylalkynes from Propiolic Acid Using PalladiumCatalyzed Decarboxylative Coupling. J. Org. Chem., 2010, 75, 6244-6251.

3. Bruker SMART V5.630 and SAINT-PLUS V6.45, Bruker-Nonius Analytical X-ray Systems Inc.: Madison, Wisconsin, USA 2003. SADABS, Empirical absorption correction program, Bruker AXS Inc., Madison, Wisconsin, USA 1997.

4. Sheldrick G M, Acta Crystallogr 64A (2008) 112. 
ag $-2-77$
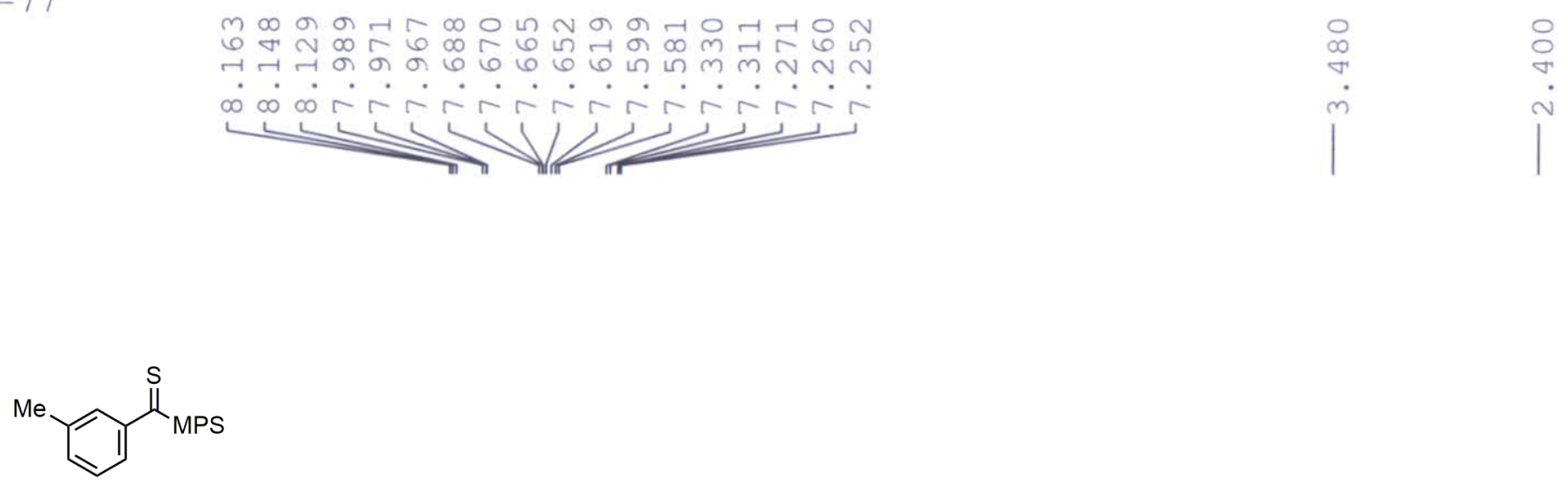

1I, ${ }^{1} \mathrm{H}$ NMR (400 MHz, $\mathrm{CDCl}_{3}$ )

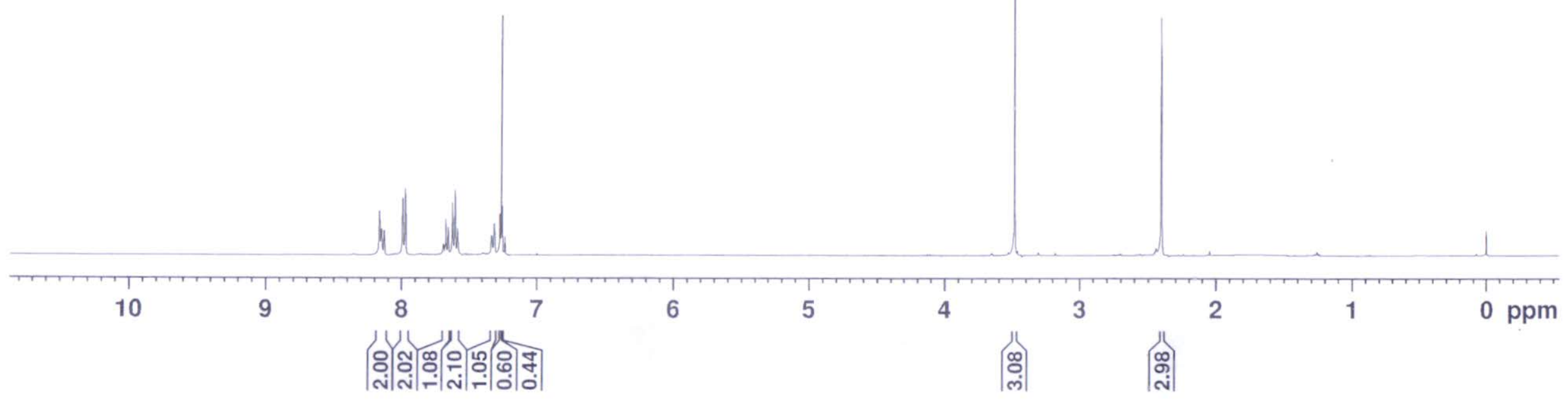

S5 
ag $-2-77$

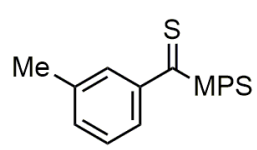

1I, ${ }^{13} \mathrm{C}\left\{{ }^{1} \mathrm{H}\right\}$ NMR $\left(101 \mathrm{MHz}, \mathrm{CDCl}_{3}\right)$

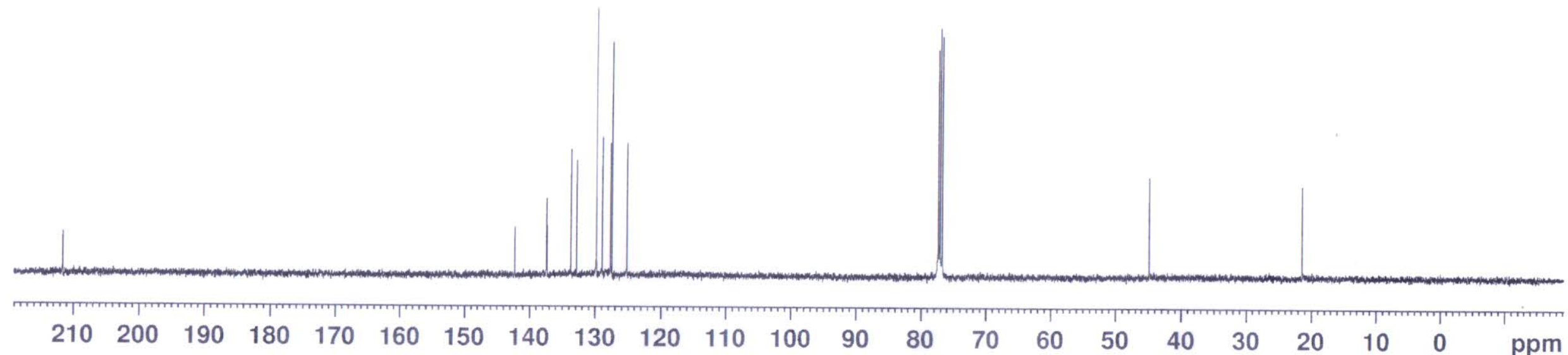


MJ-106-15

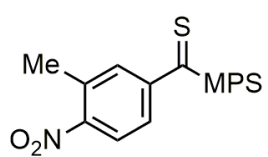

1n, ${ }^{1} \mathrm{H}$ NMR $\left(400 \mathrm{MHz}, \mathrm{CDCl}_{3}\right)$

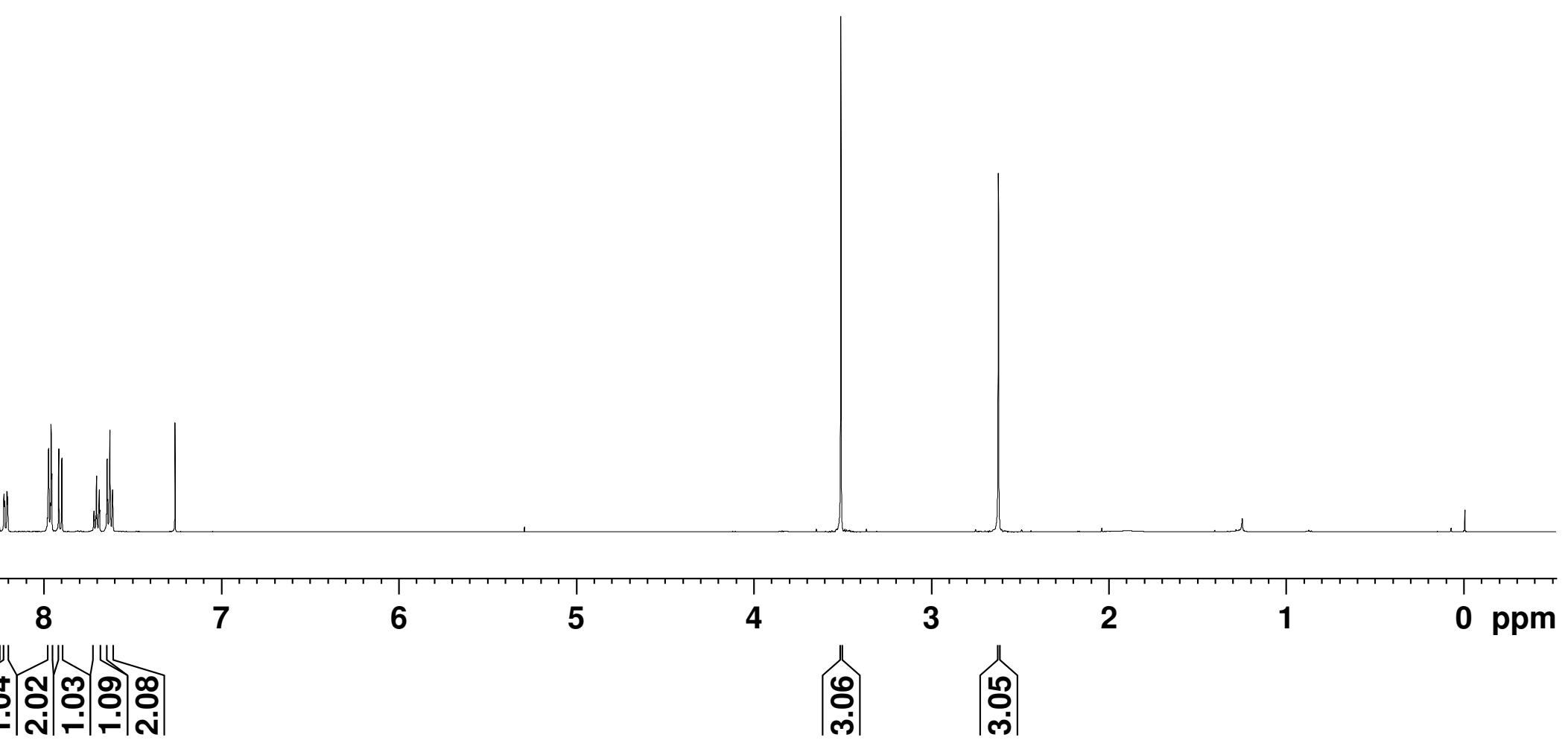


MJ-106-15

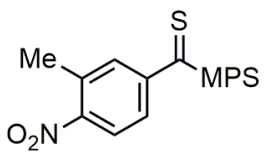

1n, ${ }^{13} \mathrm{C}\left\{{ }^{1} \mathrm{H}\right\} \mathrm{NMR}\left(101 \mathrm{MHz}, \mathrm{CDCl}_{3}\right)$

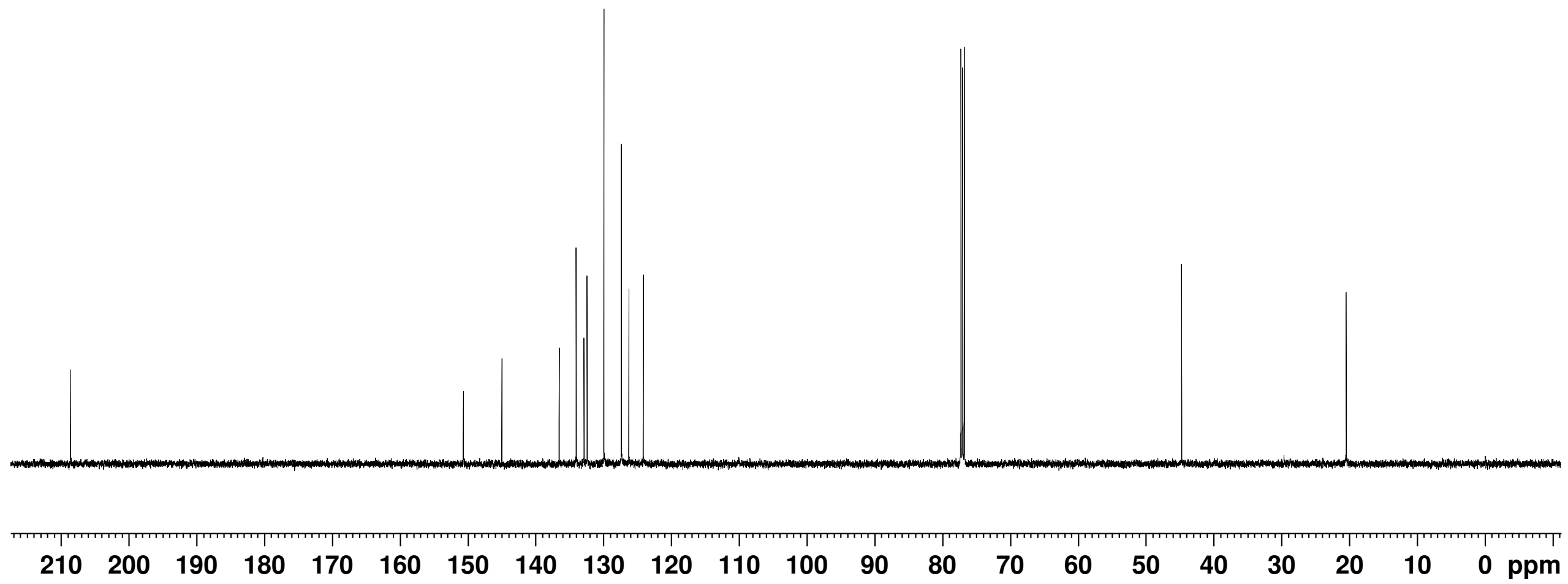


AS-4-192

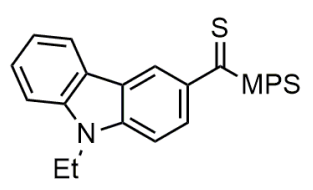

10, ${ }^{1} \mathrm{H}$ NMR $\left(400 \mathrm{MHz}, \mathrm{CDCl}_{3}\right)$

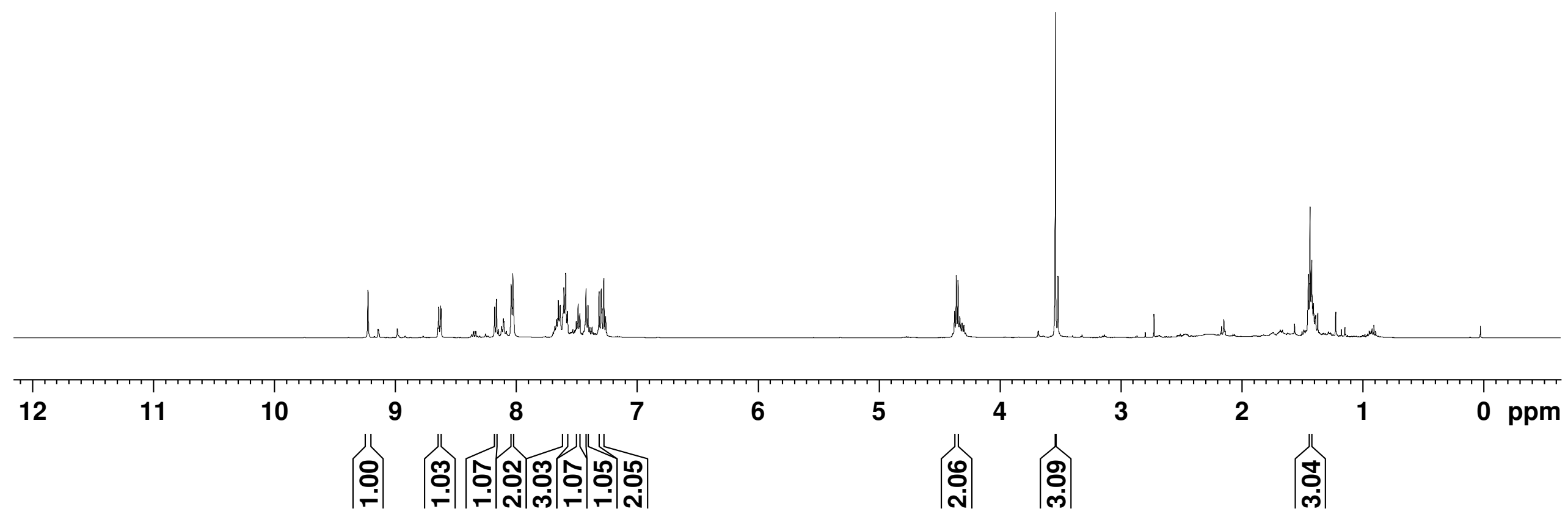


$\stackrel{8}{\circ}$

AS-4-192

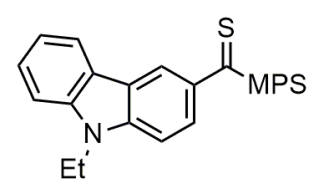

1o, ${ }^{13} \mathrm{C}\left\{{ }^{1} \mathrm{H}\right\} \mathrm{NMR}\left(101 \mathrm{MHz}, \mathrm{CDCl}_{3}\right)$
ฉ

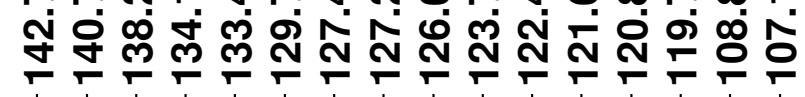

มฺุ

Nヘ

$1 /$

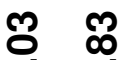

กั่

$\stackrel{\infty}{\infty}$

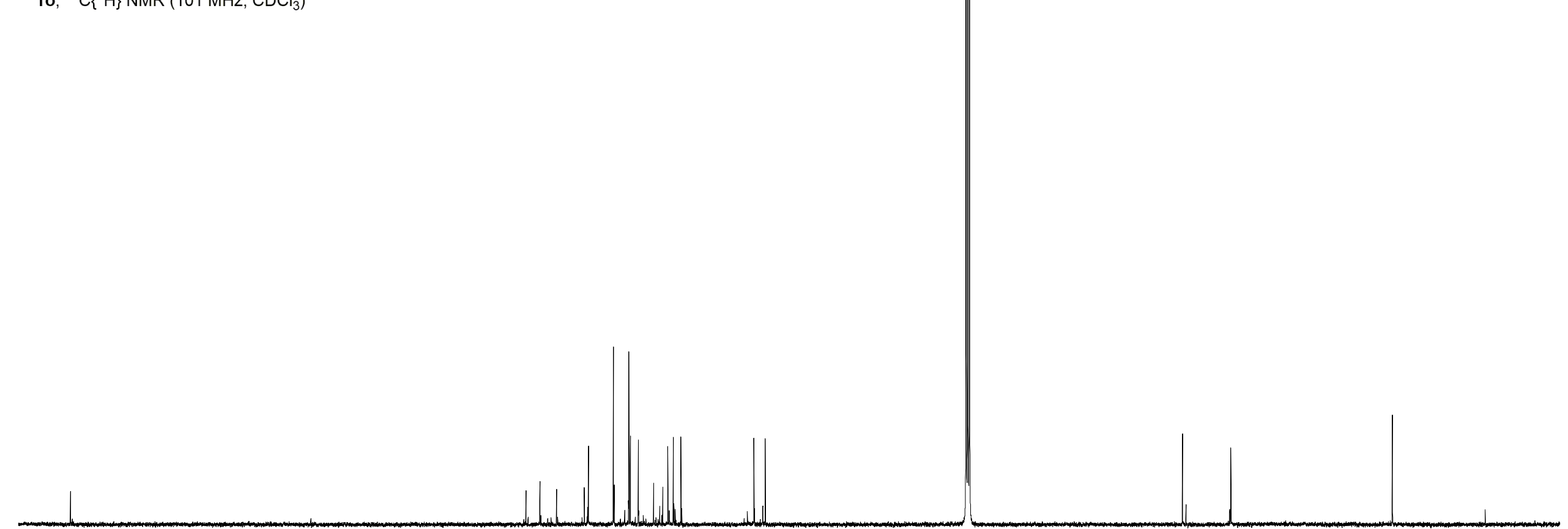

$\begin{array}{lllllllllllllllllllllll}210 & 200 & 190 & 180 & 170 & 160 & 150 & 140 & 130 & 120 & 110 & 100 & 90 & 80 & 70 & 60 & 50 & 40 & 30 & 20 & 10 & 0 & \text { ppm }\end{array}$ 

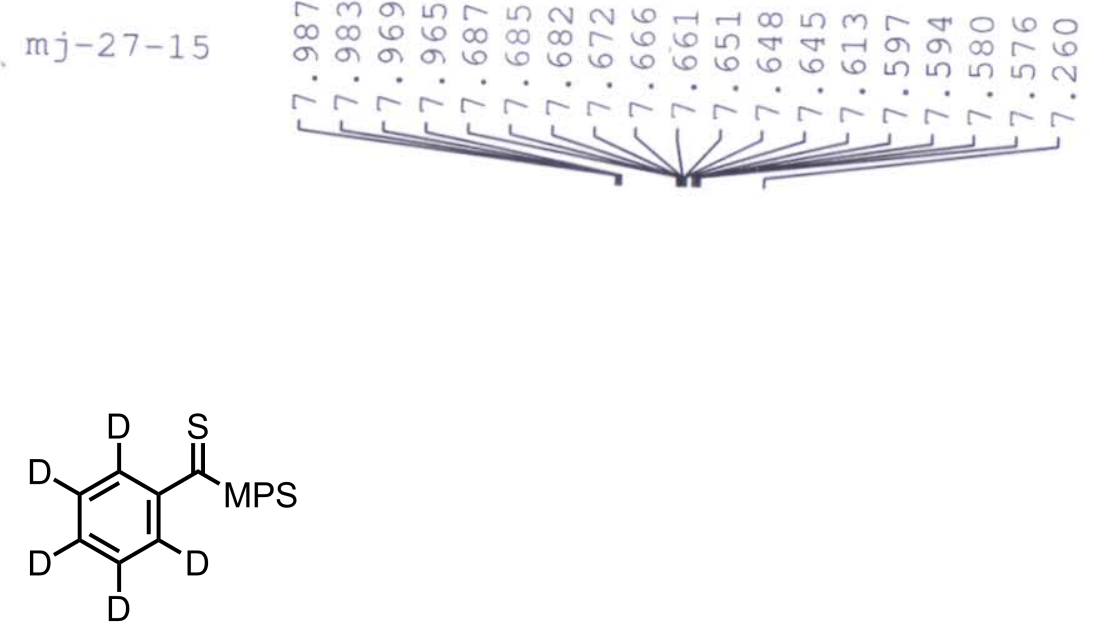

1d-D5, ${ }^{1} \mathrm{H}$ NMR, $400 \mathrm{MHz}, \mathrm{CDCl}_{3}$

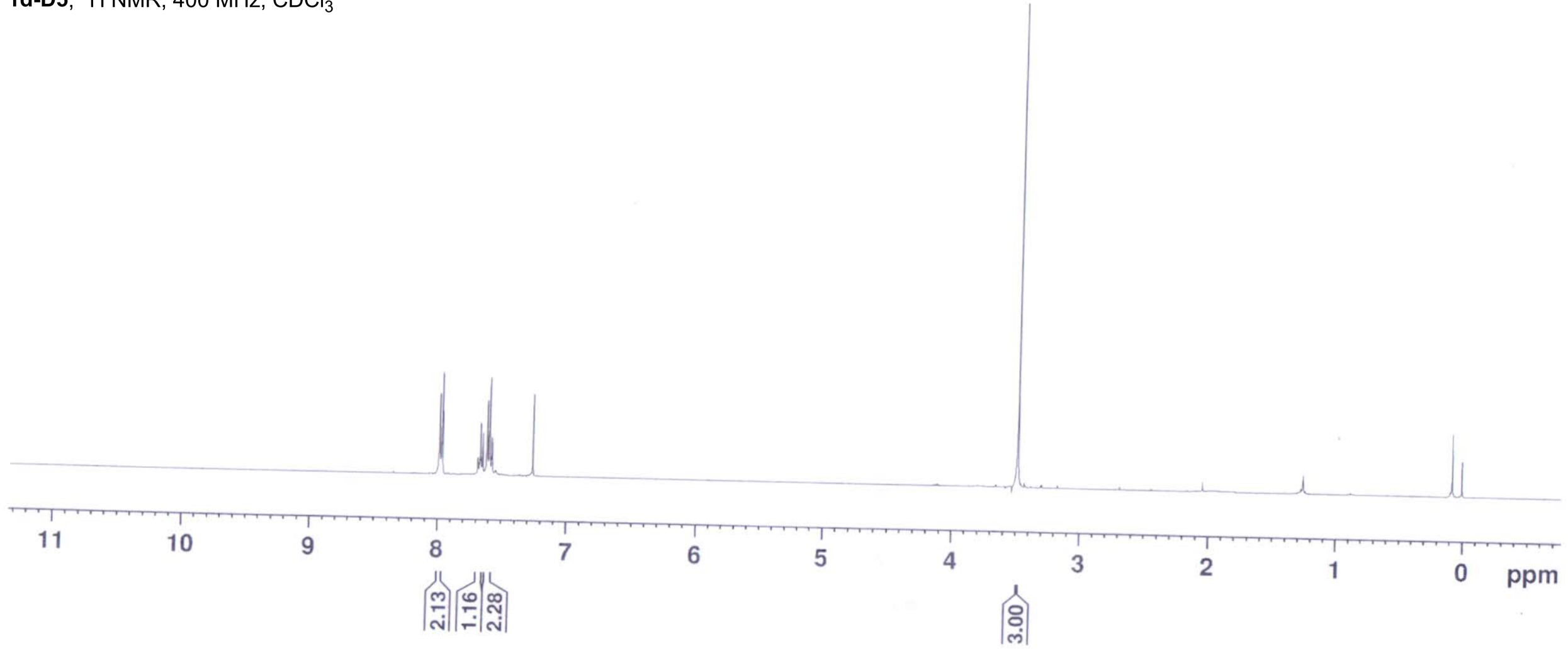




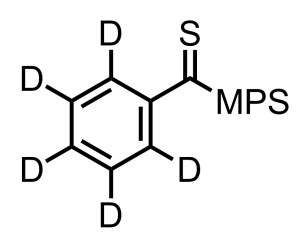

1d-D5, ${ }^{13} \mathrm{C}\left\{{ }^{1} \mathrm{H}\right\} \mathrm{NMR}, 101 \mathrm{MHz}, \mathrm{CDCl}_{3}$

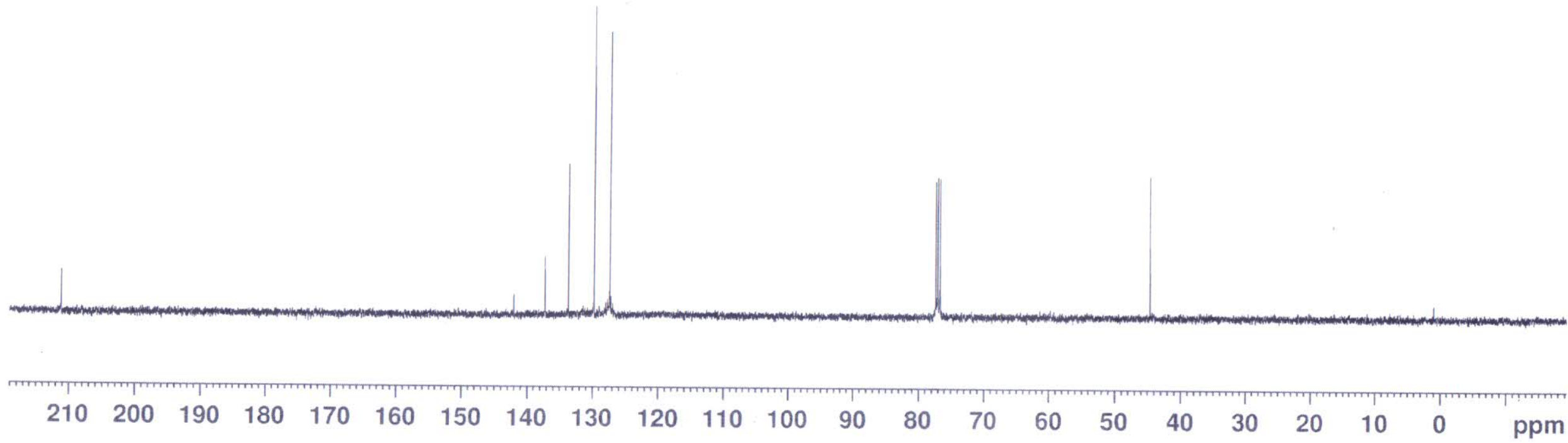




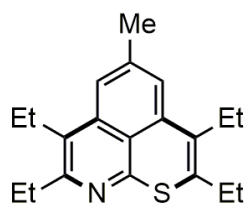

3a, Scheme 1

$\mathrm{H}$ NMR $\left(400 \mathrm{MHz}, \mathrm{CDCl}_{3}\right)$

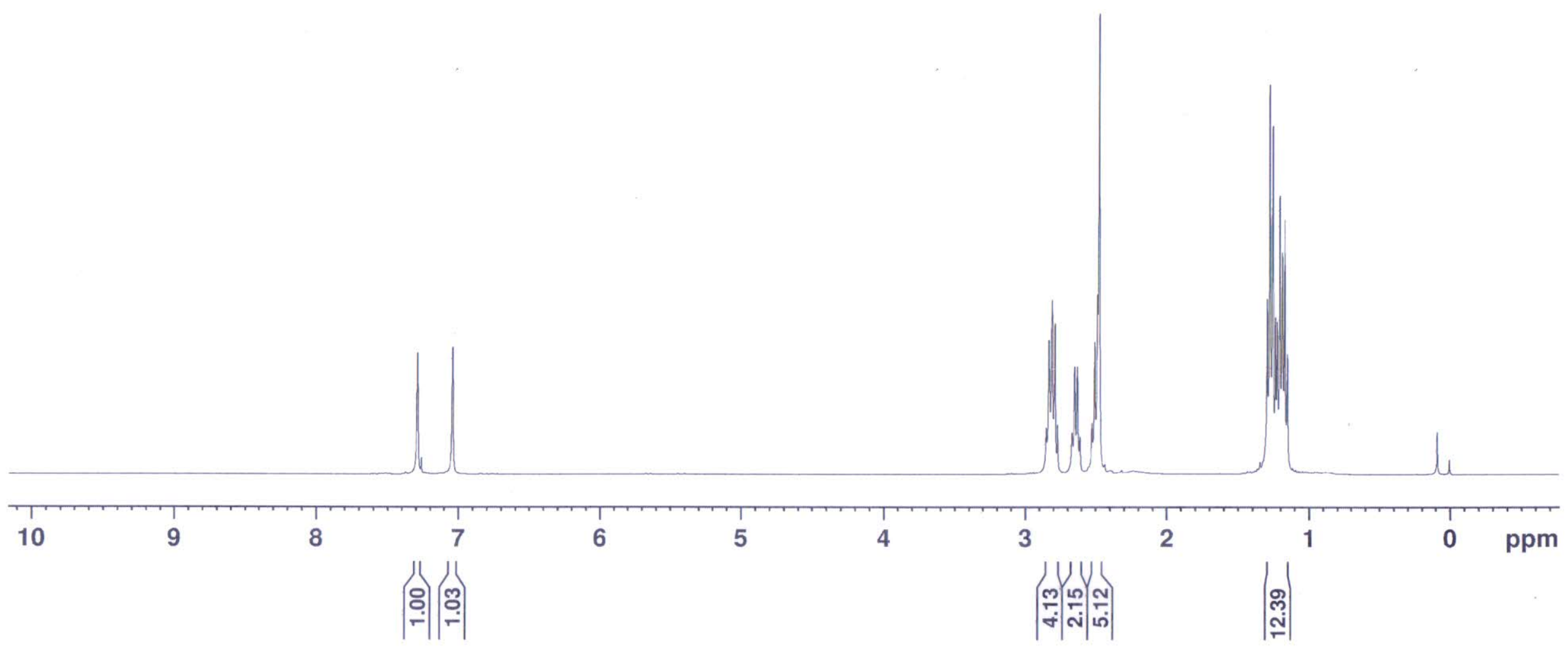



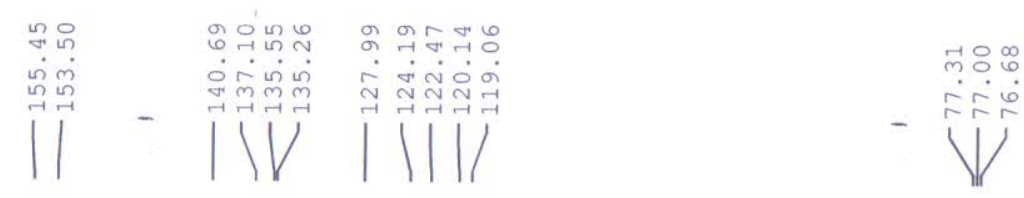

MJ-3-14

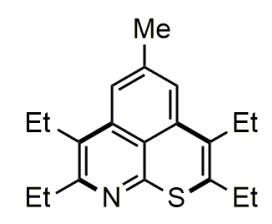

3a, Scheme 1

${ }^{13} \mathrm{C}\left\{{ }^{1} \mathrm{H}\right\}$ NMR $\left(101 \mathrm{MHz}, \mathrm{CDCl}_{3}\right)$

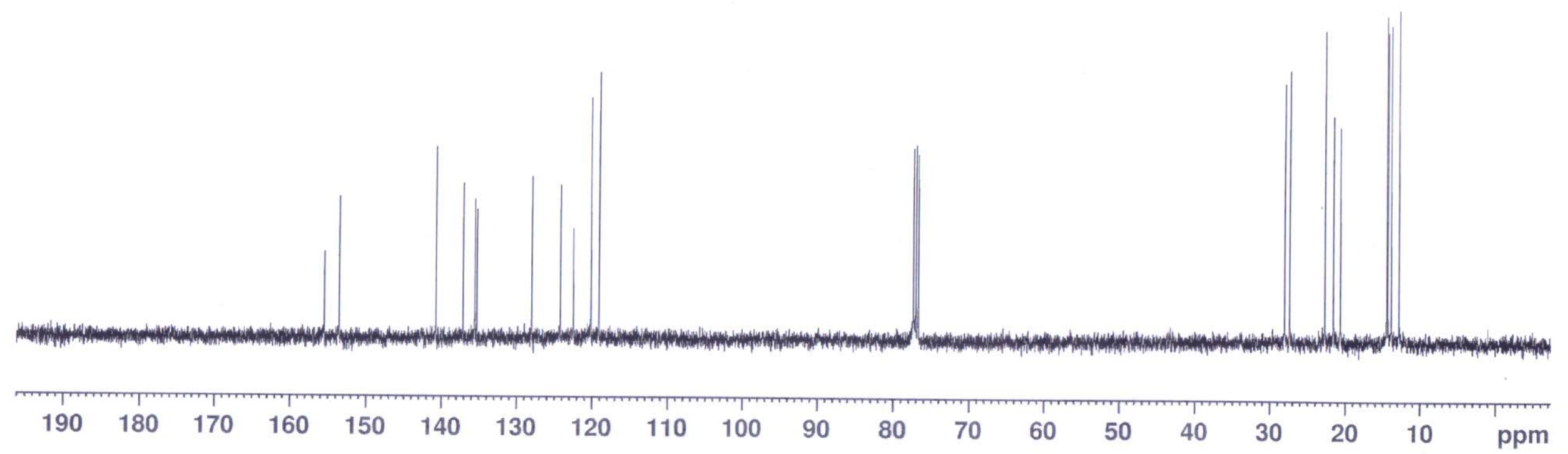




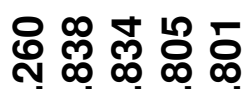

....

(1)
の

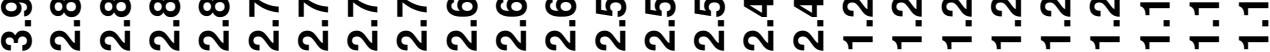

AS-25

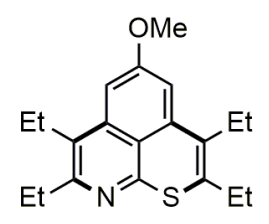

3b, Scheme 1

${ }^{1} \mathrm{H} \mathrm{NMR}\left(500 \mathrm{MHz}, \mathrm{CDCl}_{3}\right)$

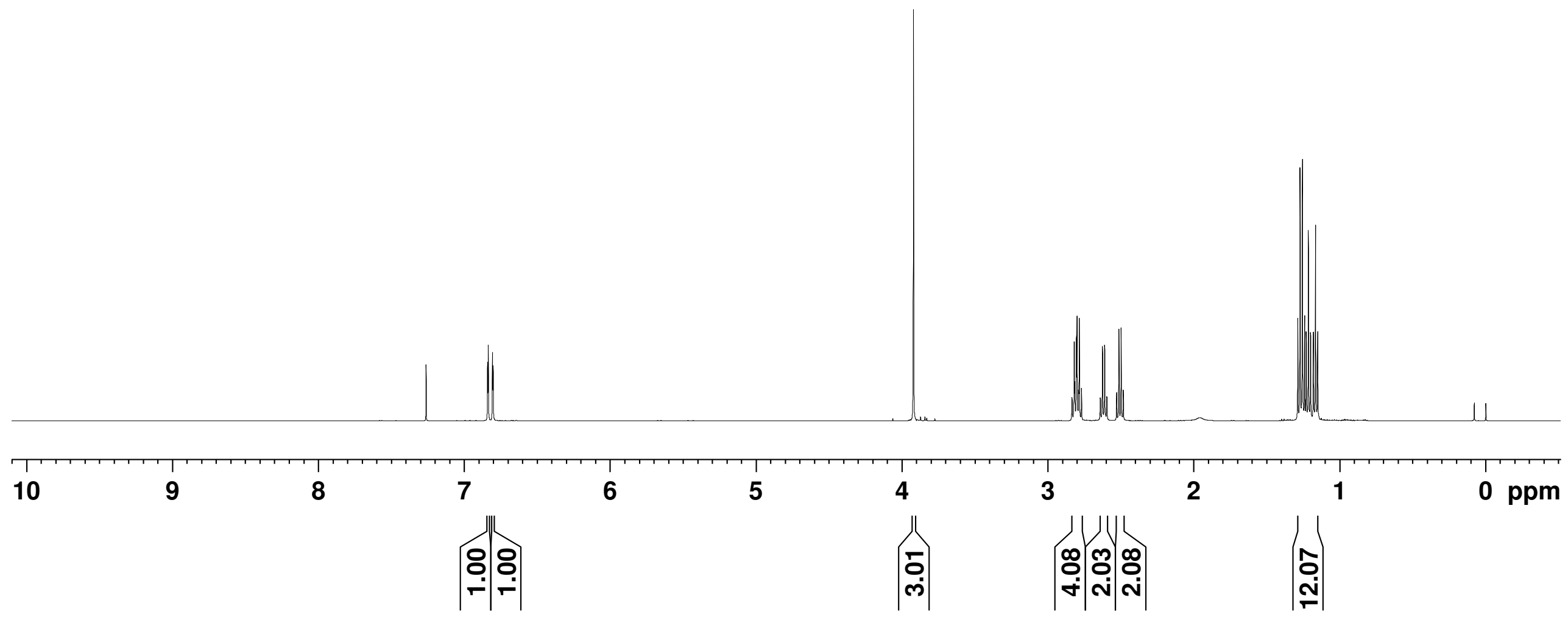




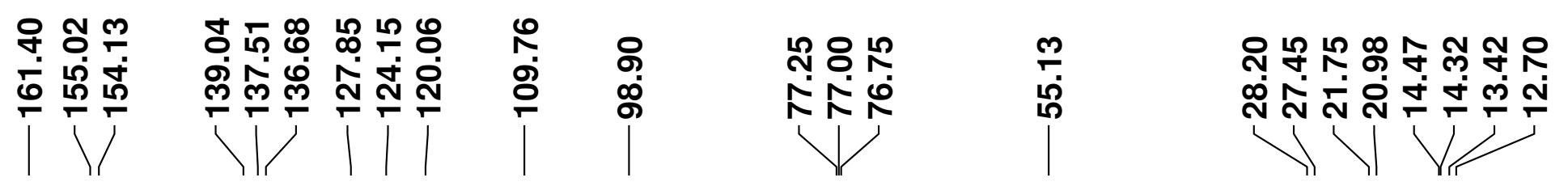

AS-25

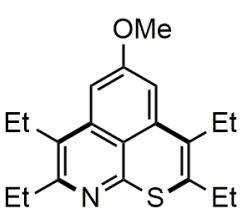

3b, Scheme 1

${ }^{13} \mathrm{C}\left\{{ }^{1} \mathrm{H}\right\}$ NMR $\left(126 \mathrm{MHz}, \mathrm{CDCl}_{3}\right)$
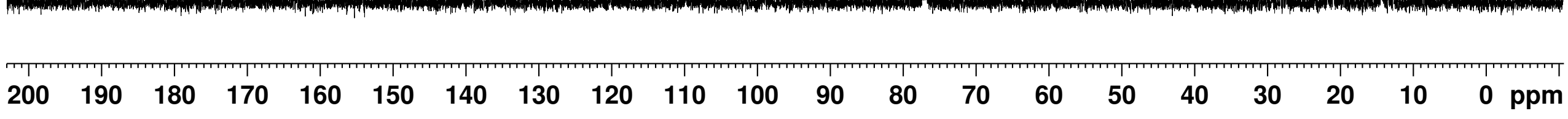


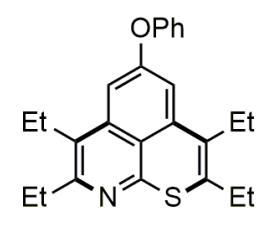

3c, Scheme

${ }^{1} \mathrm{H} \mathrm{NMR}\left(400 \mathrm{MHz}, \mathrm{CDCl}_{3}\right)$

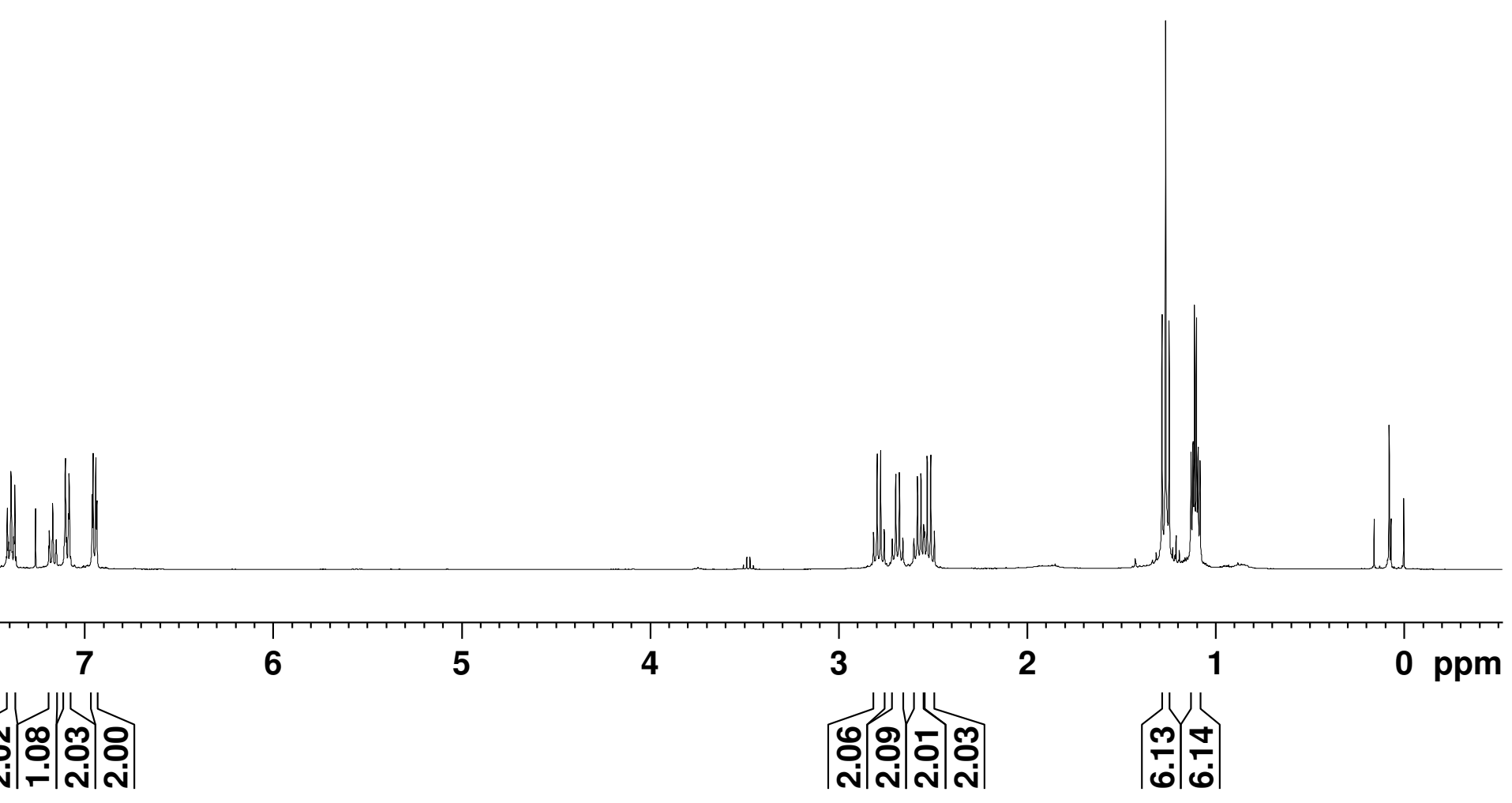




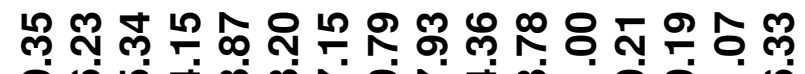

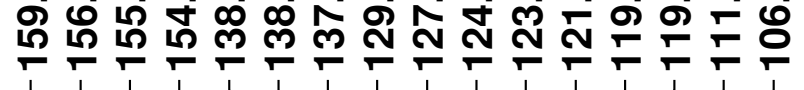
广र
ผ

ヘト゚

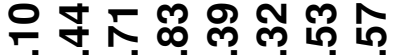

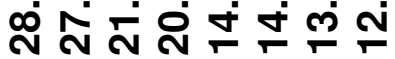

AS -40

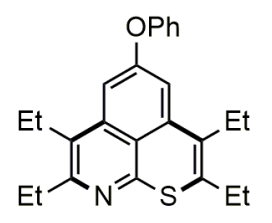

3c, Scheme 1

${ }^{13} \mathrm{C}\left\{{ }^{1} \mathrm{H}\right\}$ NMR $\left(126 \mathrm{MHz}, \mathrm{CDCl}_{3}\right)$

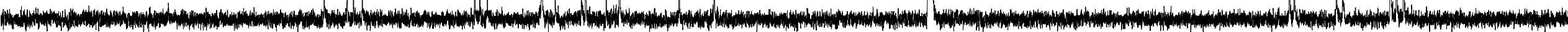

$\begin{array}{llllllllllllllllllllll}200 & 190 & 180 & 170 & 160 & 150 & 140 & 130 & 120 & 110 & 100 & 90 & 80 & 70 & 60 & 50 & 40 & 30 & 20 & 10 & 0 & \text { ppm }\end{array}$ 
$M j-23-14$

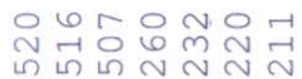

r.

$\sqrt{n}$

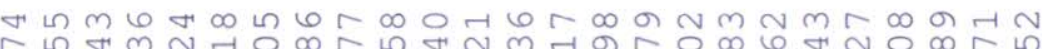

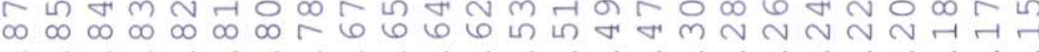
$\dot{\sim} \dot{\sim} \dot{\sim} \dot{\sim} \dot{\sim} \dot{\sim} \dot{\sim} \dot{\sim} \dot{\sim} \dot{\sim} \dot{\sim}$

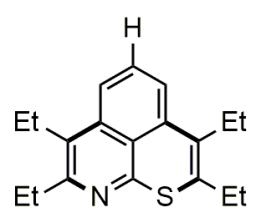

3d, Scheme 1

${ }^{1} \mathrm{H} \mathrm{NMR}\left(400 \mathrm{MHz}, \mathrm{CDCl}_{3}\right)$

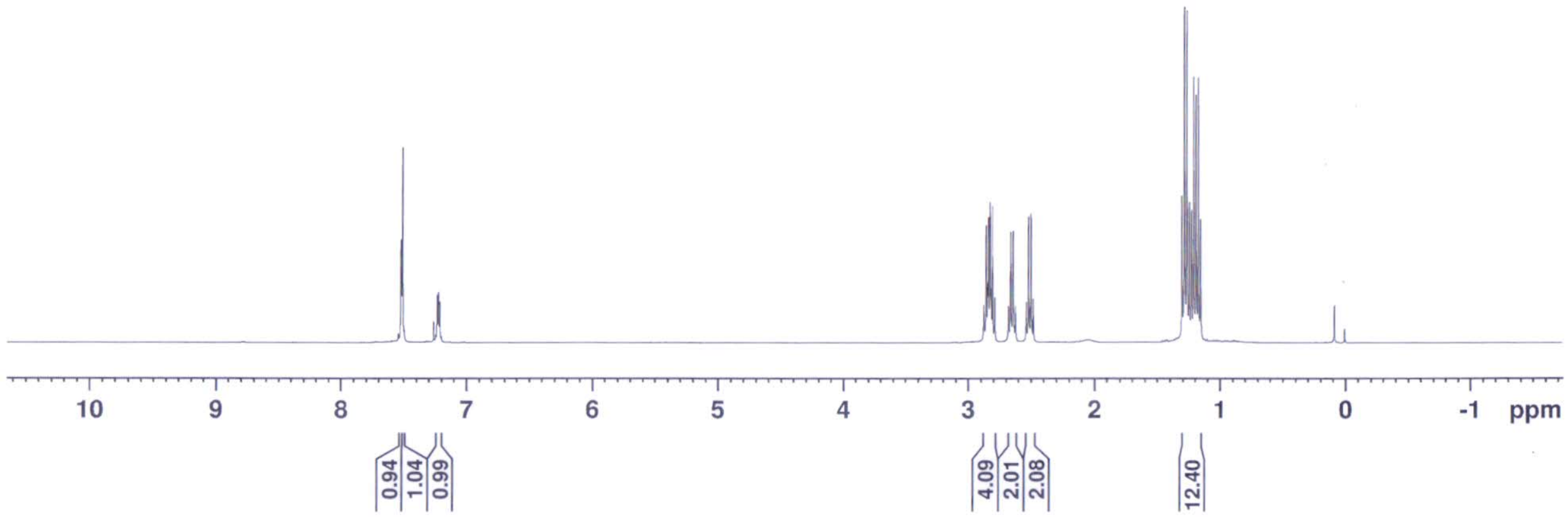




Mj-23-14

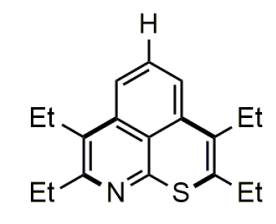

3d, Scheme 1

${ }^{13} \mathrm{C}\left\{{ }^{1} \mathrm{H}\right\}$ NMR $\left(101 \mathrm{MHz}, \mathrm{CDCl}_{3}\right)$

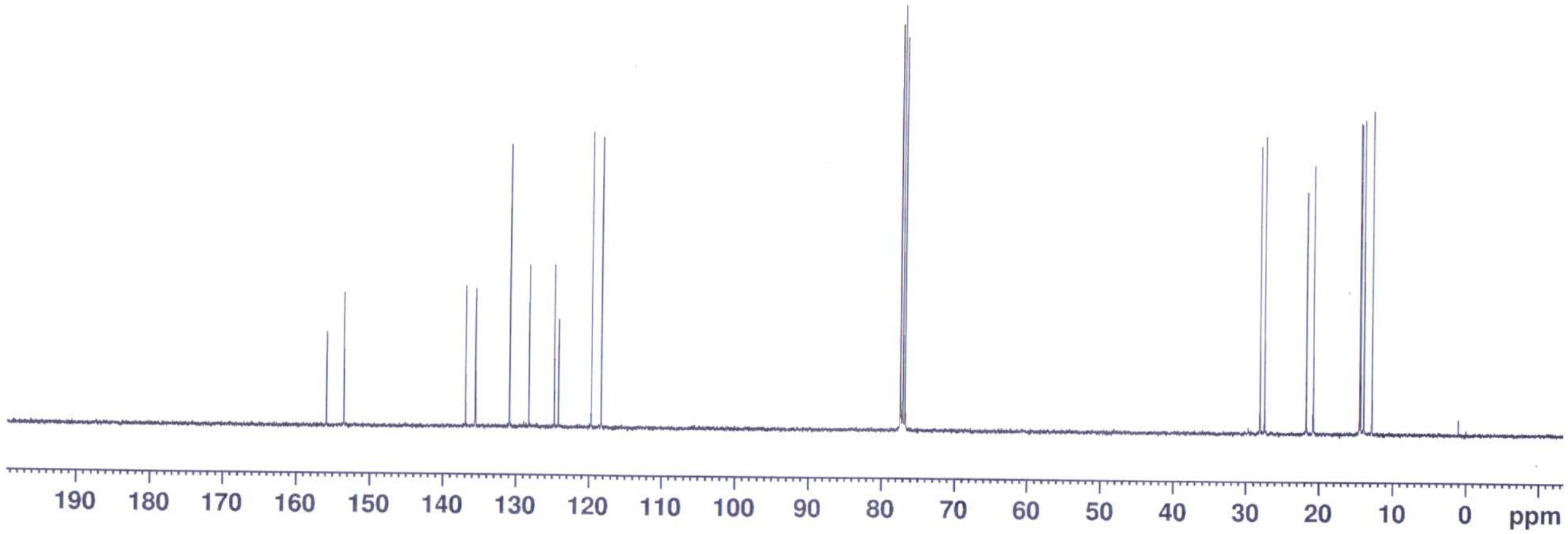


$m j-13-14$
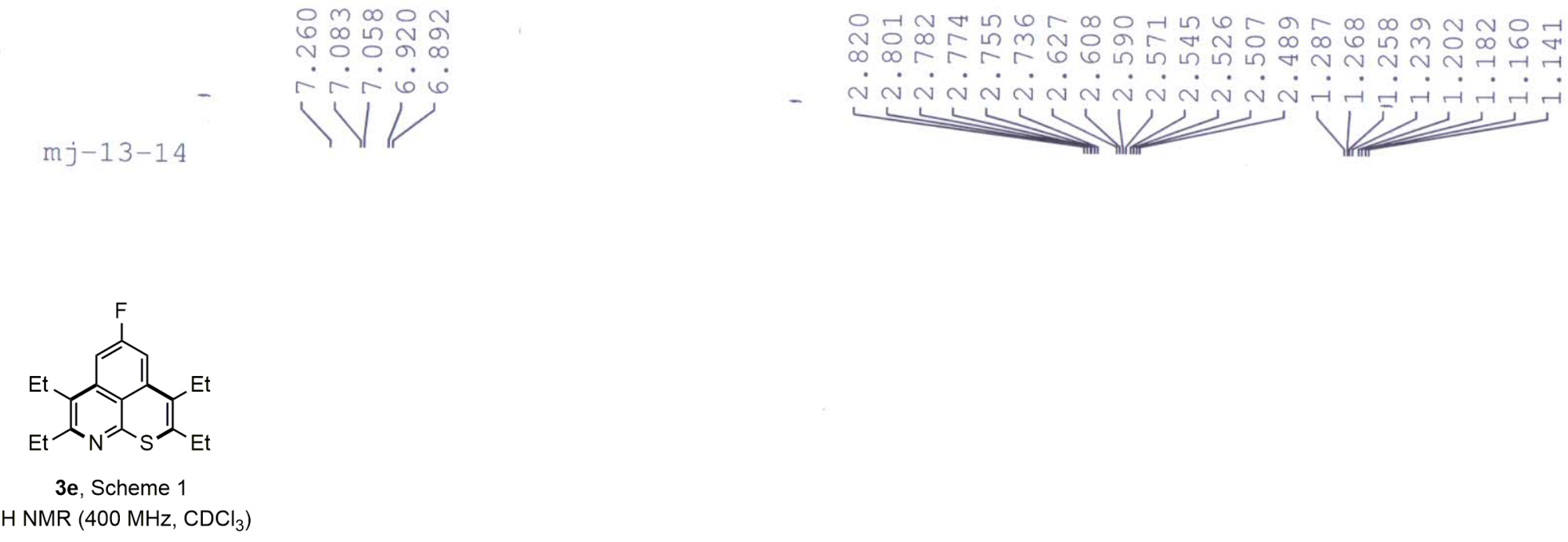

${ }^{1} \mathrm{H}$ NMR $\left(400 \mathrm{MHz}, \mathrm{CDCl}_{3}\right)$

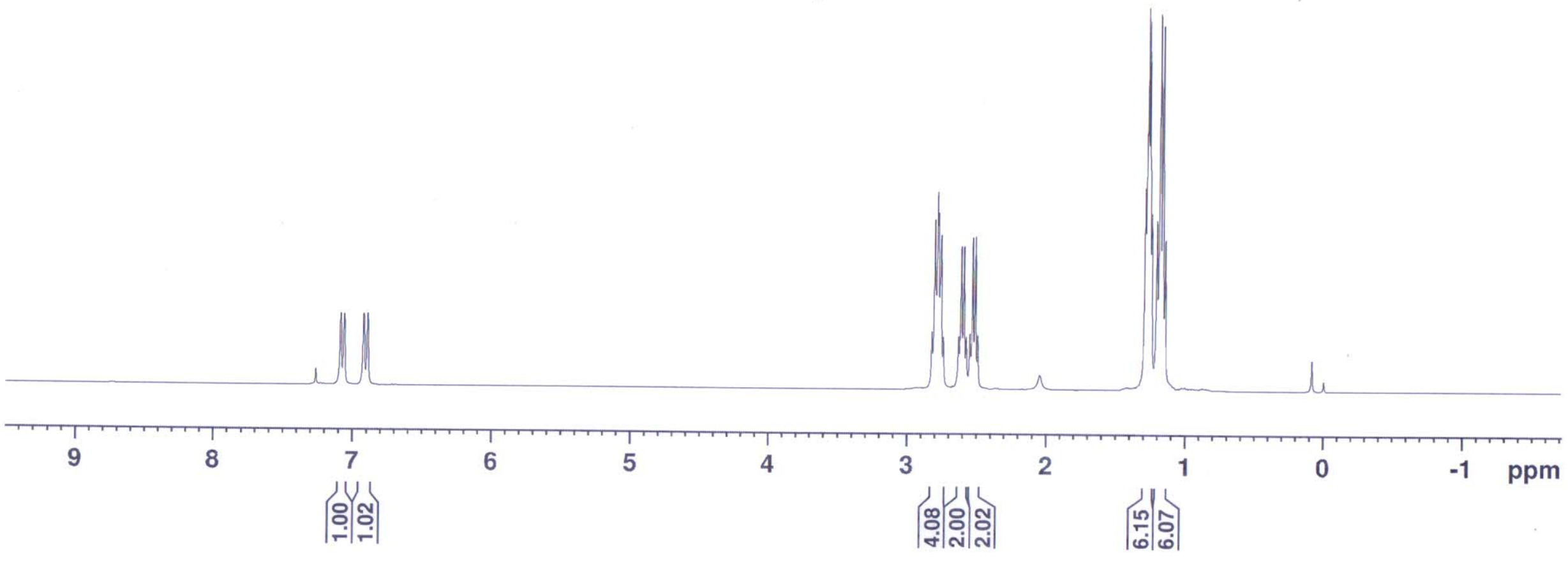

S21 

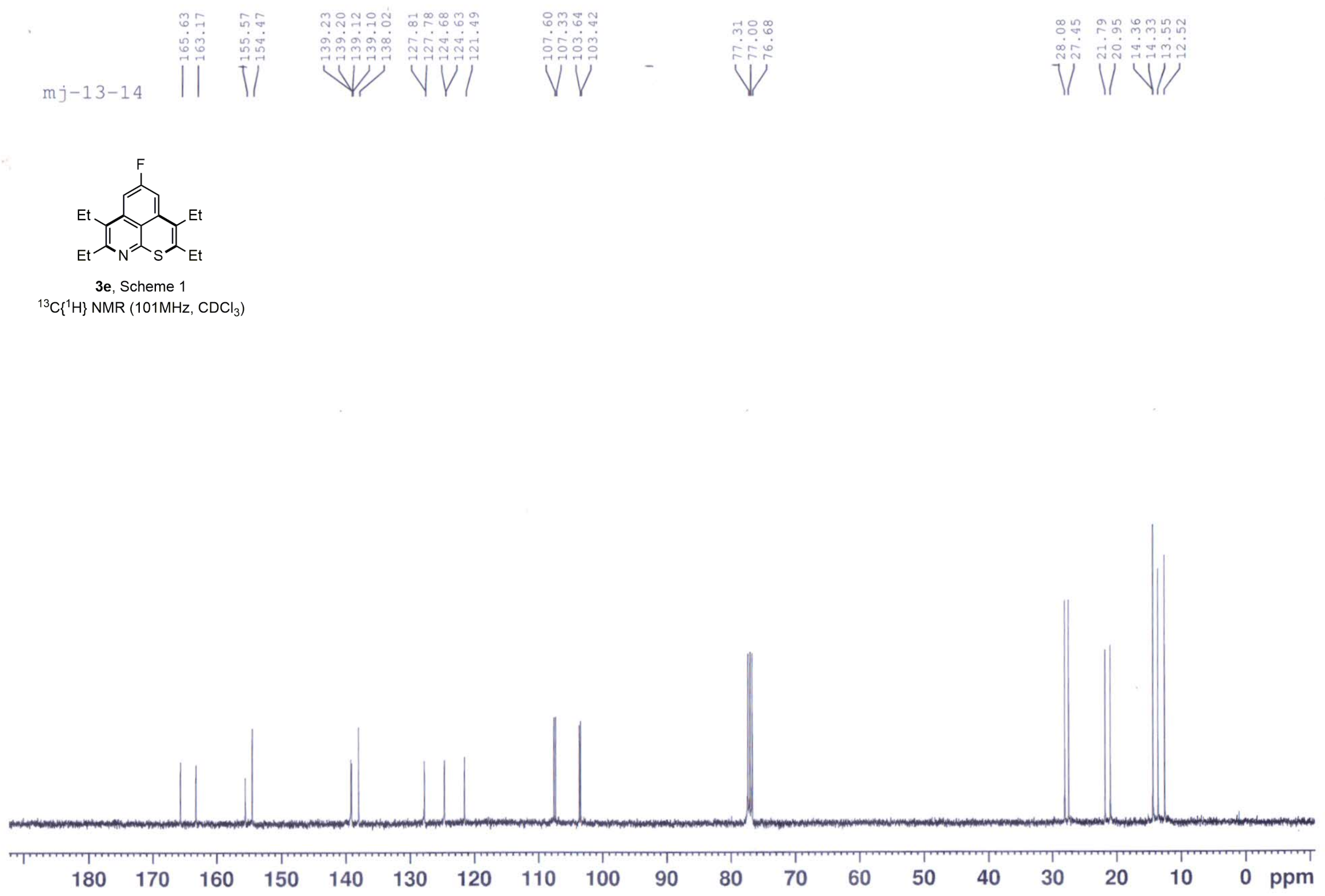


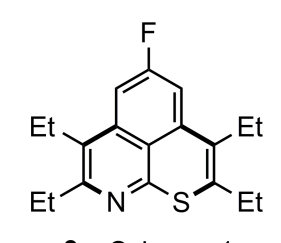

3e, Scheme 1

${ }^{19} \mathrm{~F} \mathrm{NMR}\left(376 \mathrm{MHz}, \mathrm{CDCl}_{3}\right)$

\begin{tabular}{|l|lllllllll|l|l|}
\hline 0 & -20 & -40 & -60 & -80 & -100 & -120 & -140 & -160 & -180 & -200 & $\mathrm{ppm}$
\end{tabular}




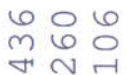

.

$m j-16-14$

iii
ॠ

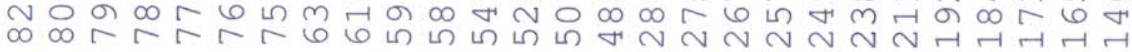

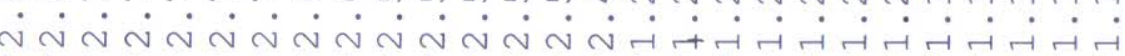

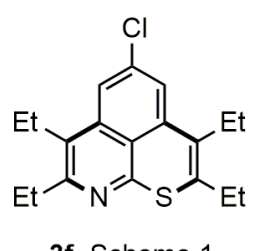

3f, Scheme 1

${ }^{1} \mathrm{H} \mathrm{NMR}\left(400 \mathrm{MHz}, \mathrm{CDCl}_{3}\right)$

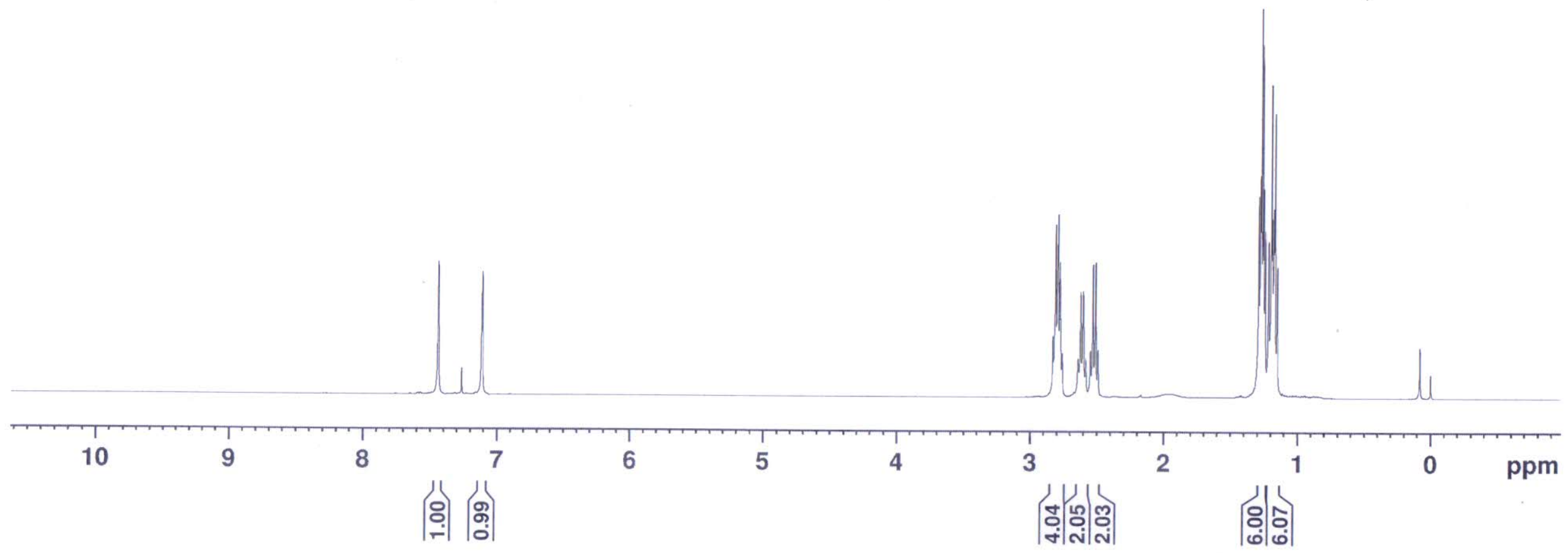




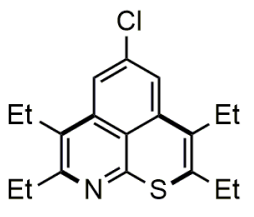

3f, Scheme 1

${ }^{13} \mathrm{C}\left\{{ }^{1} \mathrm{H}\right\}$ NMR $\left(101 \mathrm{MHz}, \mathrm{CDCl}_{3}\right)$

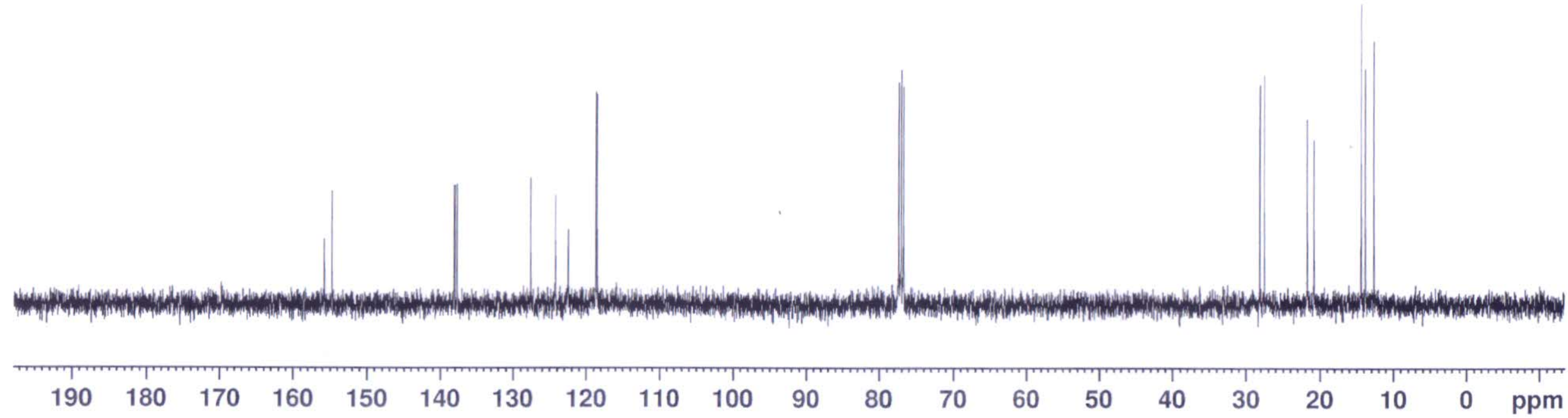


.

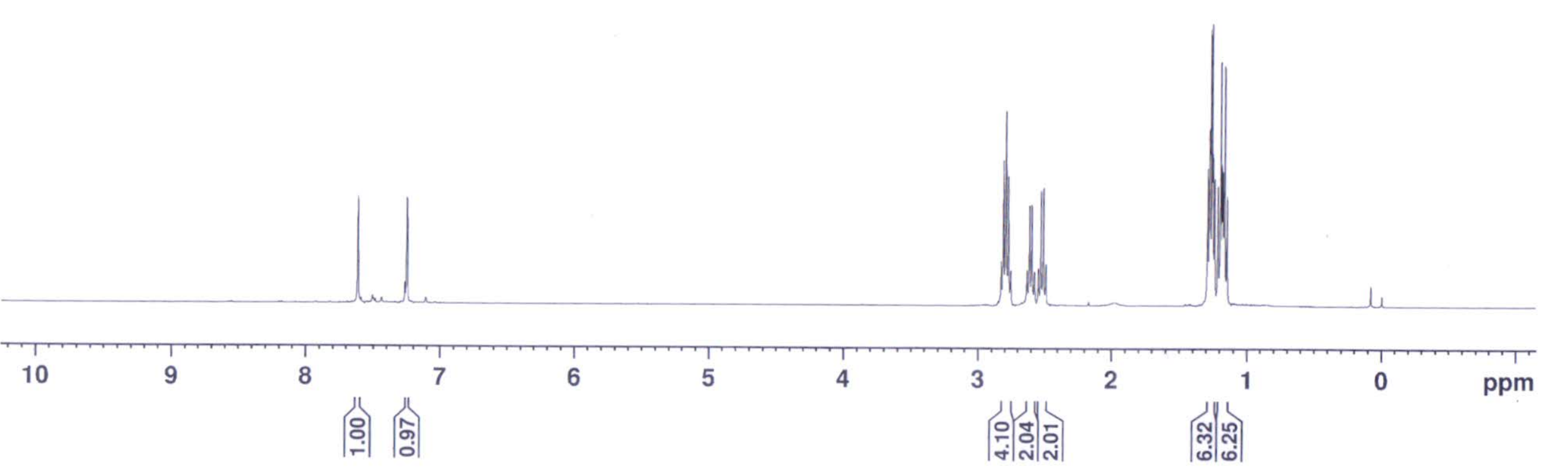



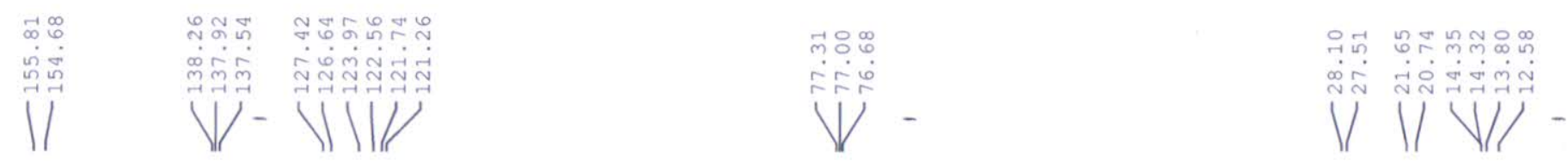

$m j-17-14$

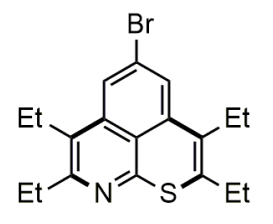

$3 g$, Scheme

${ }^{13} \mathrm{C}\left\{{ }^{1} \mathrm{H}\right\}$ NMR $\left(101 \mathrm{MHz}, \mathrm{CDCl}_{3}\right)$

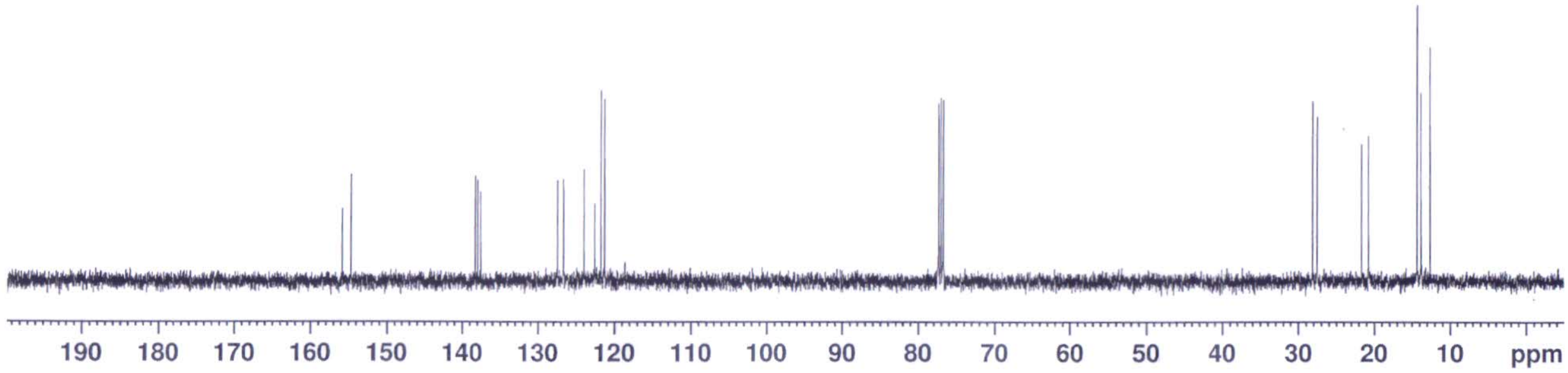




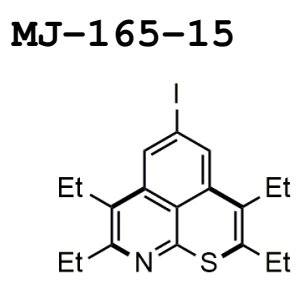

3h, Scheme 1

${ }^{1} \mathrm{H} \mathrm{NMR}\left(400 \mathrm{MHz}, \mathrm{CDCl}_{3}\right)$

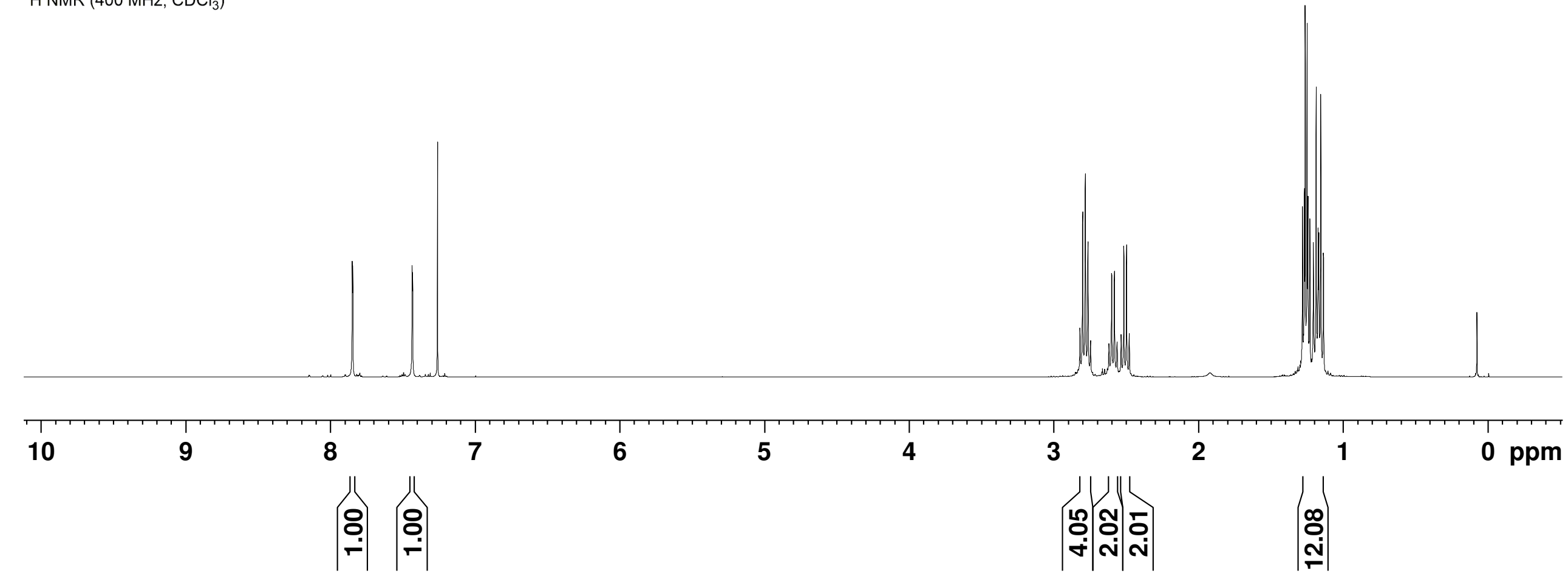




||

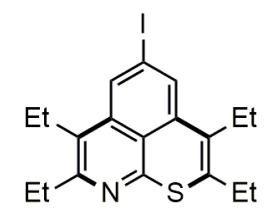

3h, Scheme 1

${ }^{13} \mathrm{C}\left\{{ }^{1} \mathrm{H}\right\} \mathrm{NMR}\left(101 \mathrm{MHz}, \mathrm{CDCl}_{3}\right)$

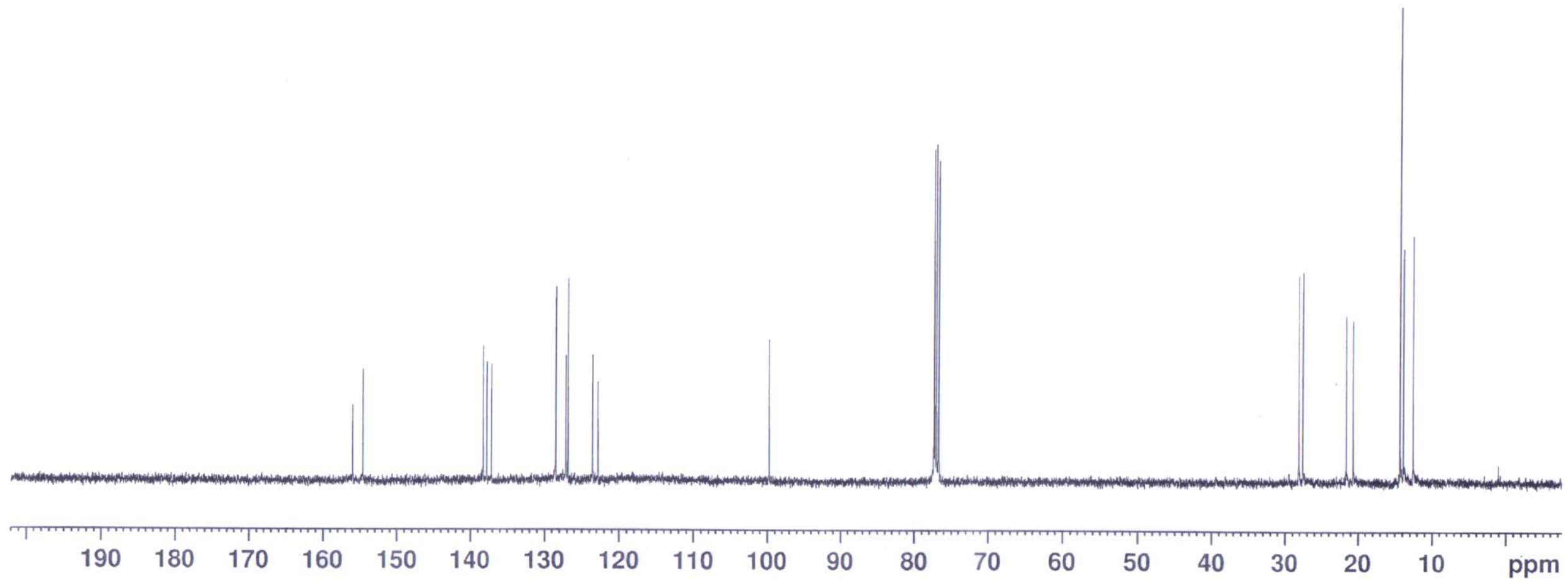


$m j-28-15$

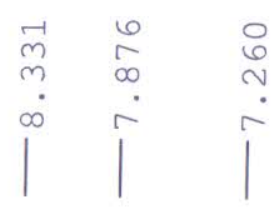

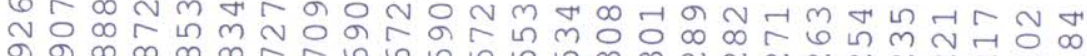
. . . . . .

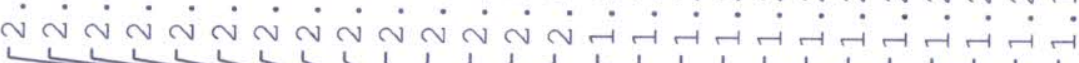

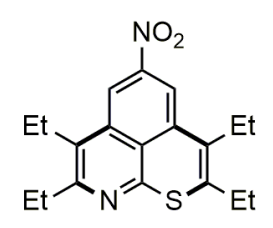

3i, Scheme 1

${ }^{1} \mathrm{H} \mathrm{NMR}\left(400 \mathrm{MHz}, \mathrm{CDCl}_{3}\right)$

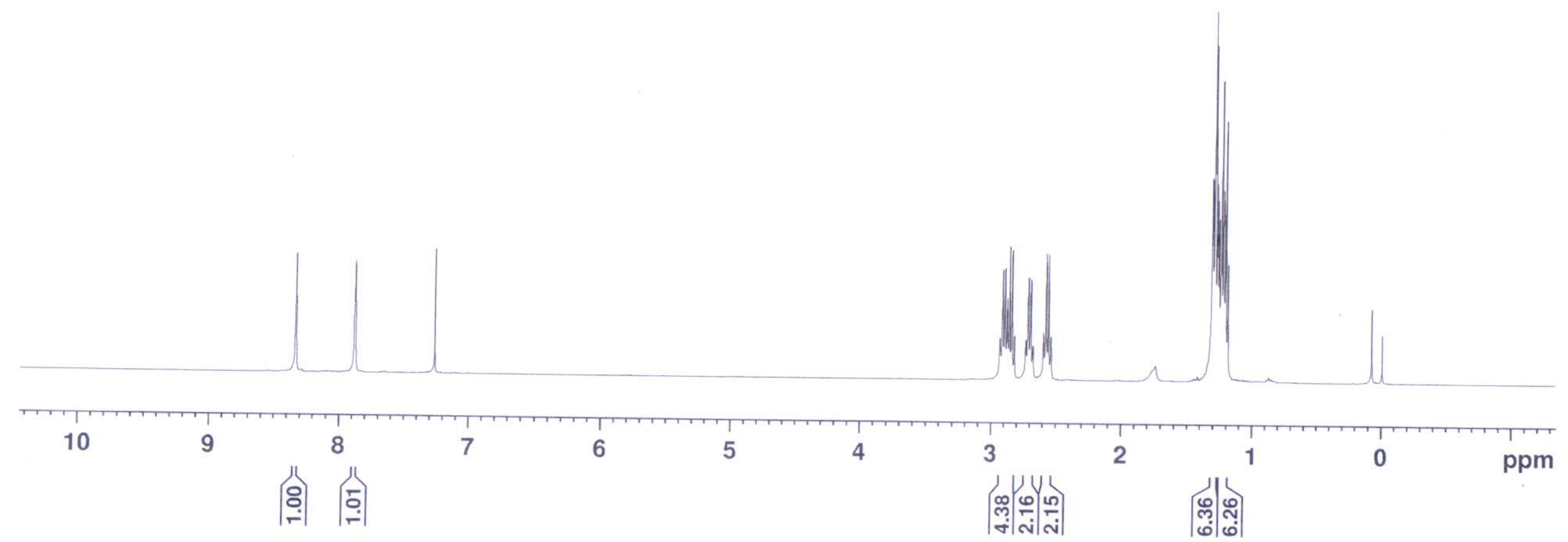



$m j-28-15$

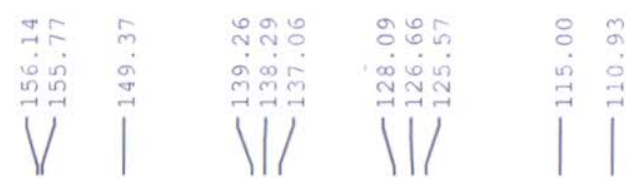

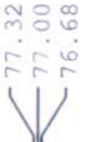

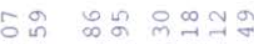
V

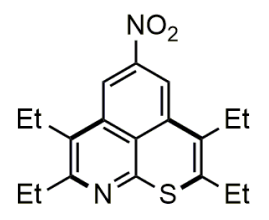

3i, Scheme 1

${ }^{13} \mathrm{C}\left\{{ }^{1} \mathrm{H}\right\} \mathrm{NMR}\left(126 \mathrm{MHz}, \mathrm{CDCl}_{3}\right)$

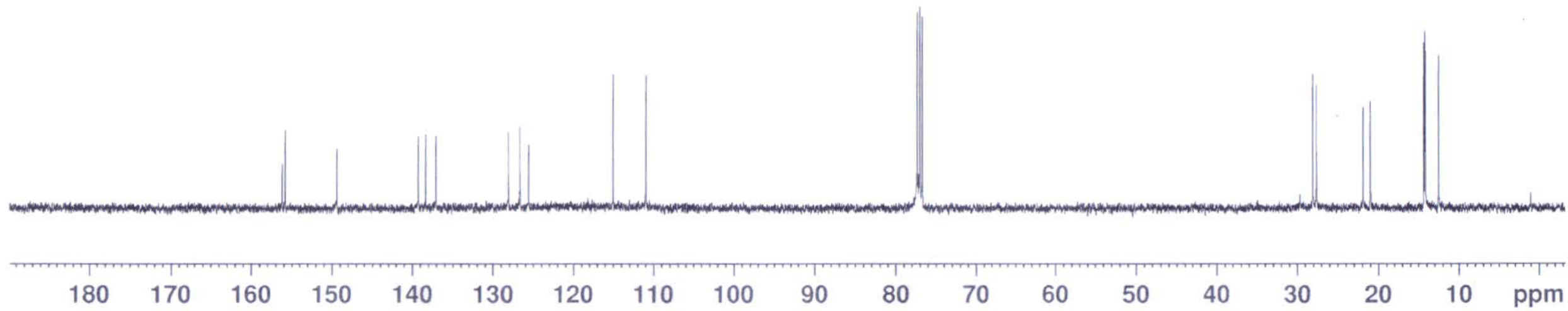




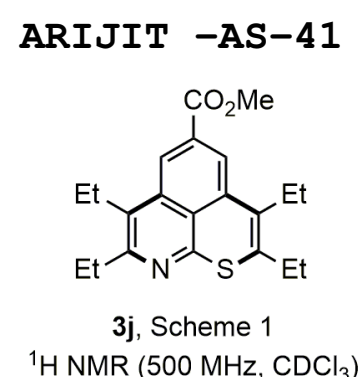

${ }^{1} \mathrm{H}$ NMR $\left(500 \mathrm{MHz}, \mathrm{CDCl}_{3}\right)$

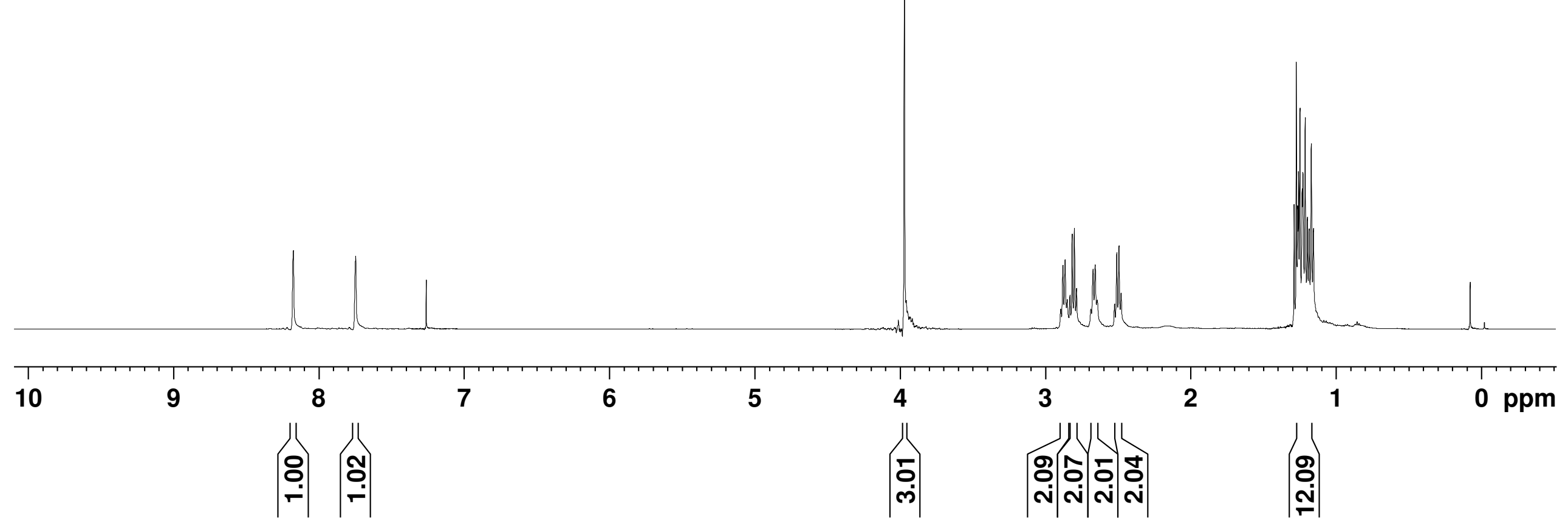




\&

ARIJIT -AS-41

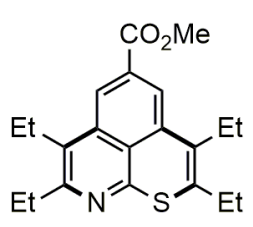

3j, Scheme 1

${ }^{13} \mathrm{C}\left\{{ }^{1} \mathrm{H}\right\}$ NMR $\left(101 \mathrm{MHz}, \mathrm{CDCl}_{3}\right)$

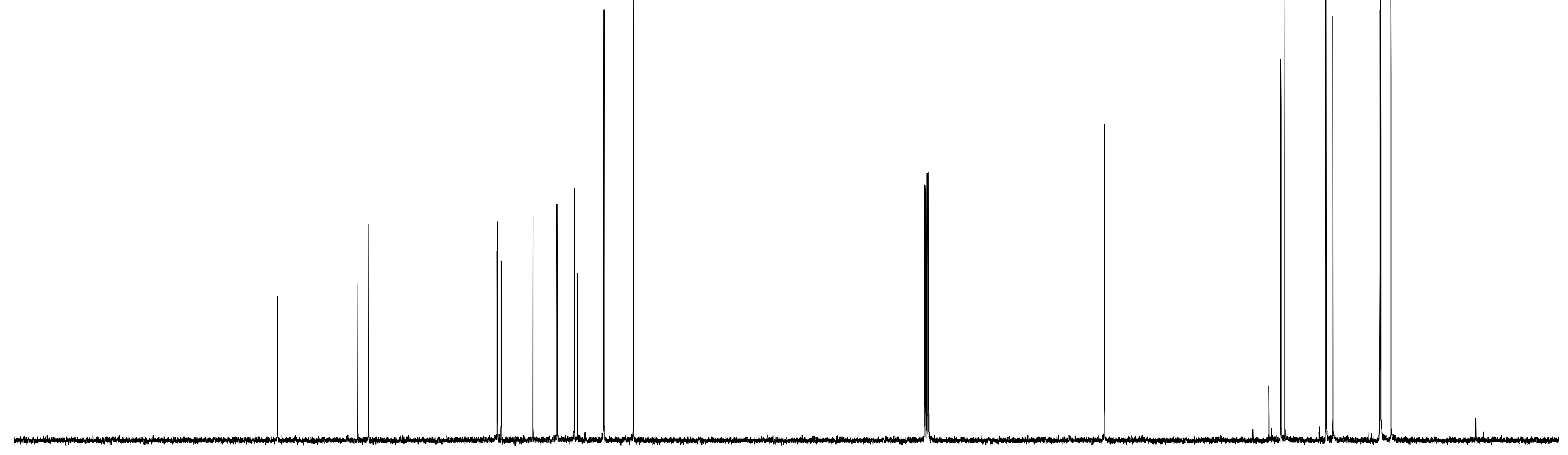

$\begin{array}{llllllllllllllllllllll}200 & 190 & 180 & 170 & 160 & 150 & 140 & 130 & 120 & 110 & 100 & 90 & 80 & 70 & 60 & 50 & 40 & 30 & 20 & 10 & 0 & \mathrm{ppm}\end{array}$ 


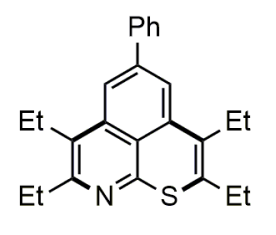

3k, Scheme 1

${ }^{1} \mathrm{H} \mathrm{NMR}\left(500 \mathrm{MHz}, \mathrm{CDCl}_{3}\right)$

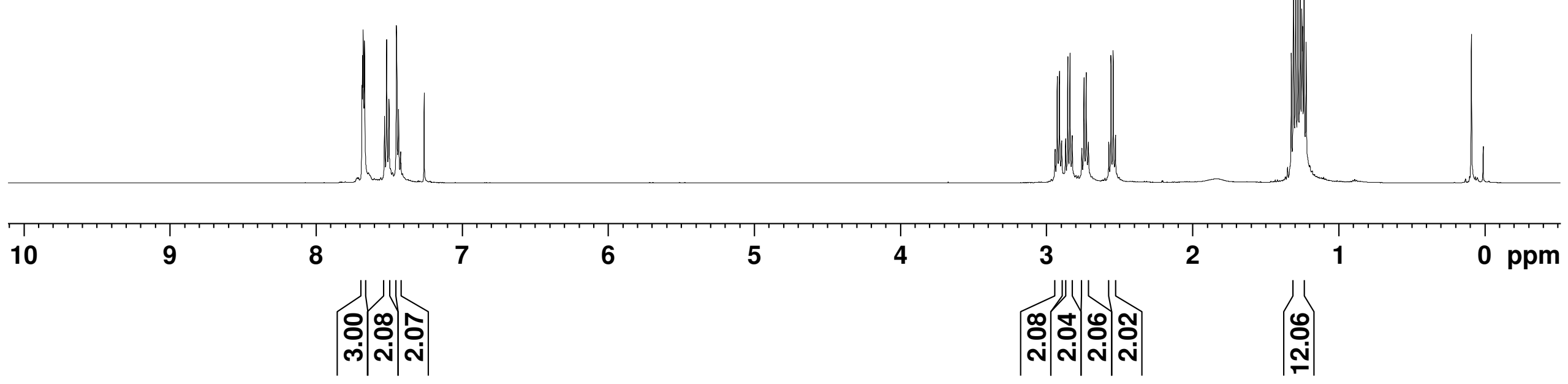




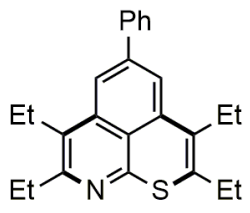

3k, Scheme 1

${ }^{13} \mathrm{C}\left\{{ }^{1} \mathrm{H}\right\} \operatorname{NMR}\left(126 \mathrm{MHz}, \mathrm{CDCl}_{3}\right)$

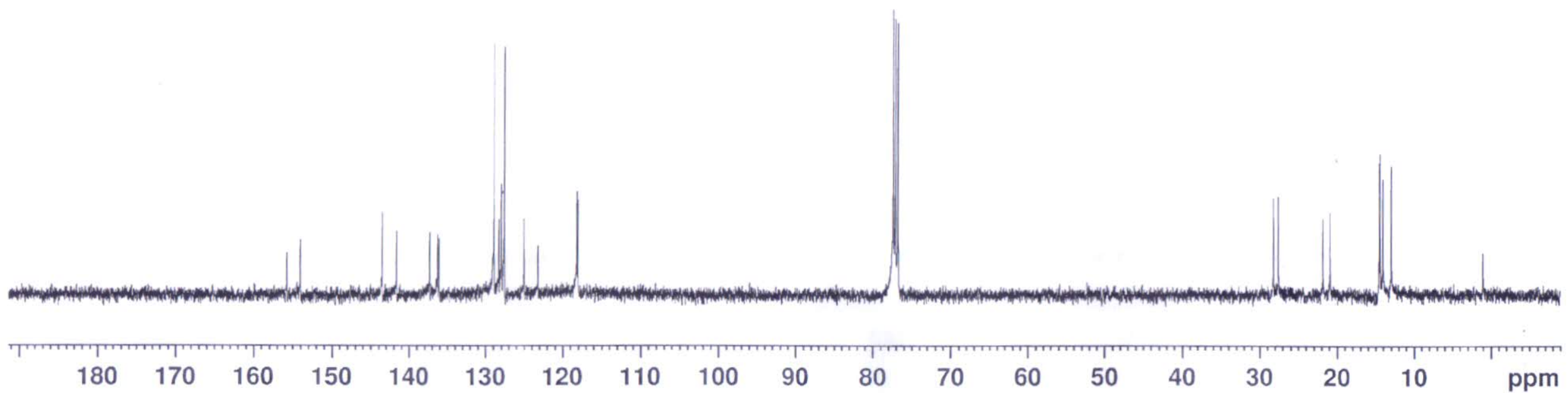


กิำ ำำ

m.

Nis

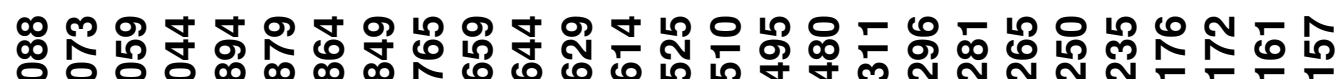

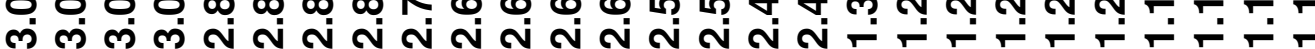

Ag-2-92

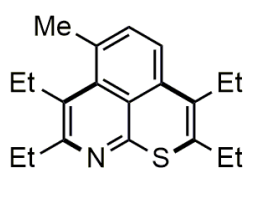

3I, Scheme 1

${ }^{1} \mathrm{H} \mathrm{NMR}\left(400 \mathrm{MHz}, \mathrm{CDCl}_{3}\right)$

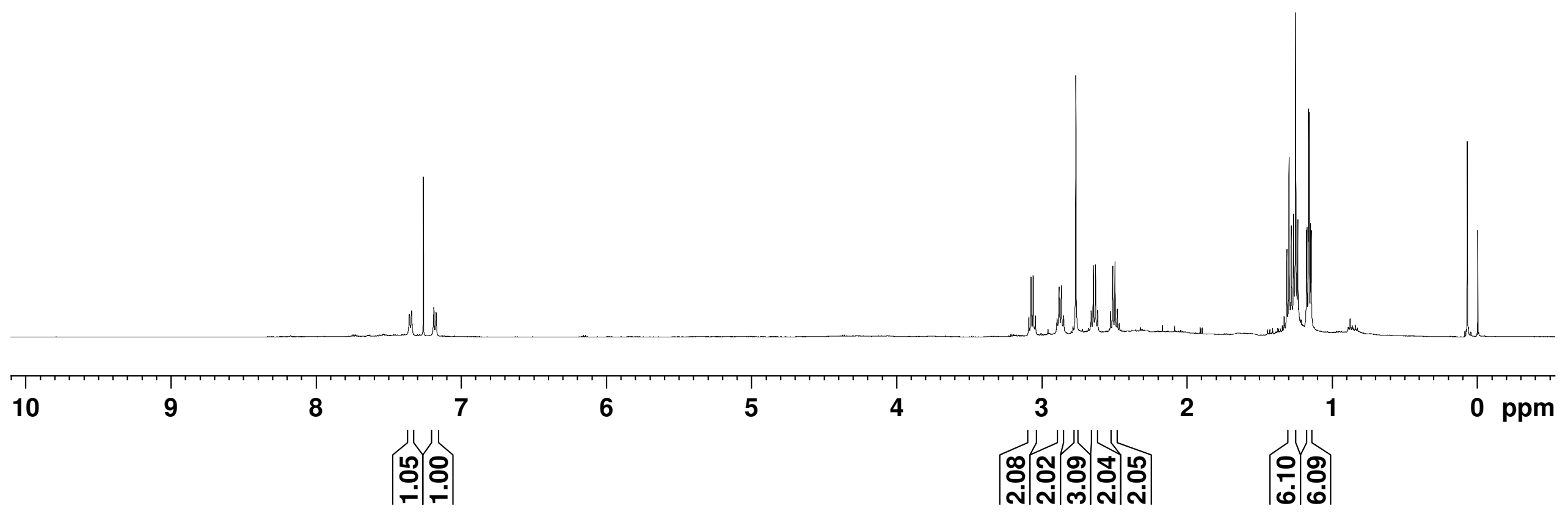




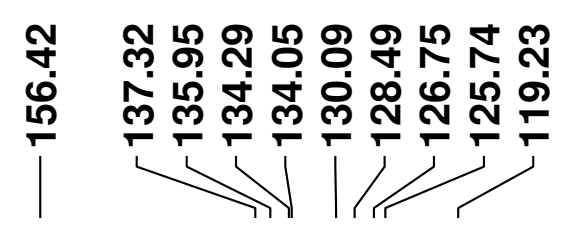

ำ 8 ำ

ヘペ゚

Ag-2-92

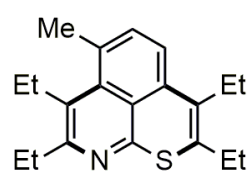

3I, Scheme 1

${ }^{13} \mathrm{C}\left\{{ }^{1} \mathrm{H}\right\} \mathrm{NMR}\left(101 \mathrm{MHz}, \mathrm{CDCl}_{3}\right)$

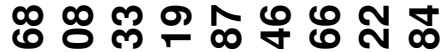
กิ่ กิ่ สู่

-

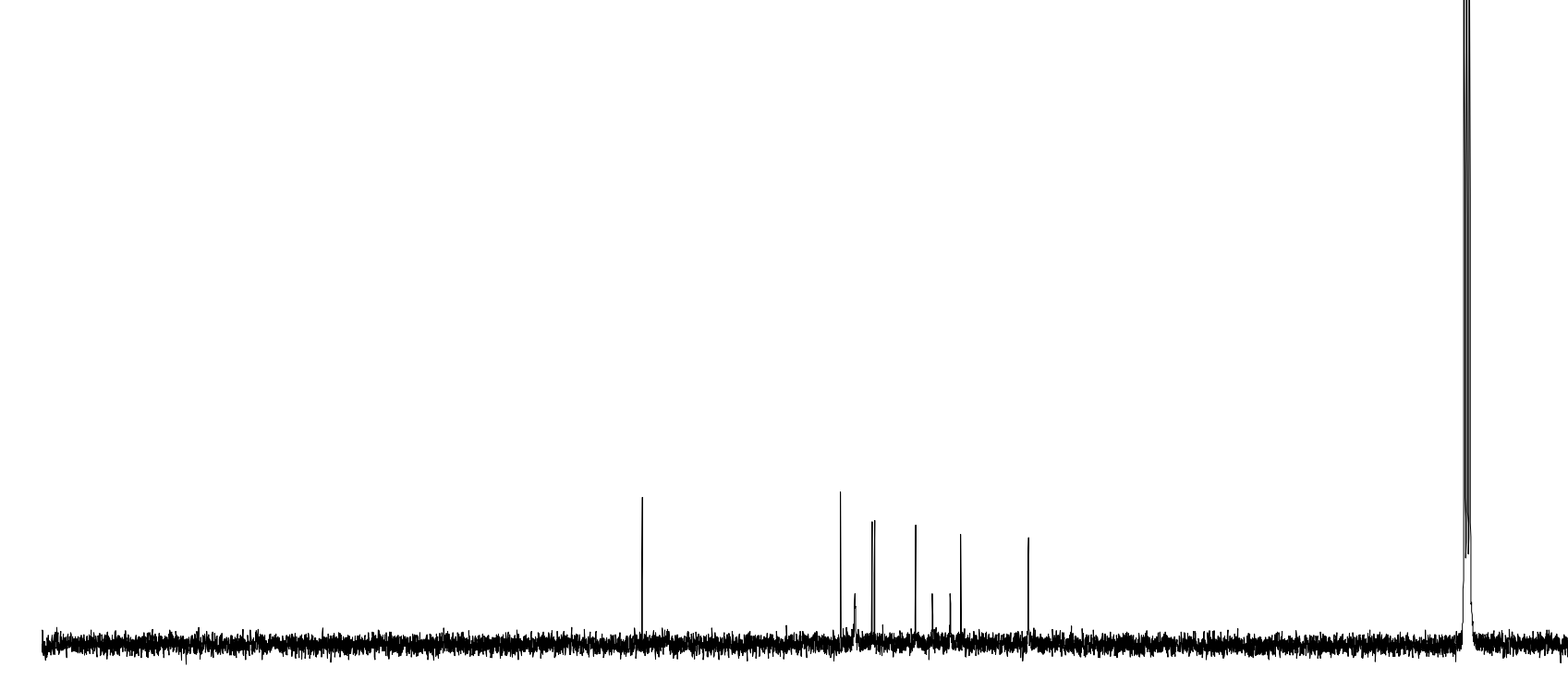
2102 
MJ-126-15

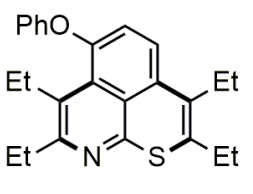

$3 \mathrm{~m}$, Scheme 1

${ }^{1} \mathrm{H} \mathrm{NMR}\left(500 \mathrm{MHz}, \mathrm{CDCl}_{3}\right)$

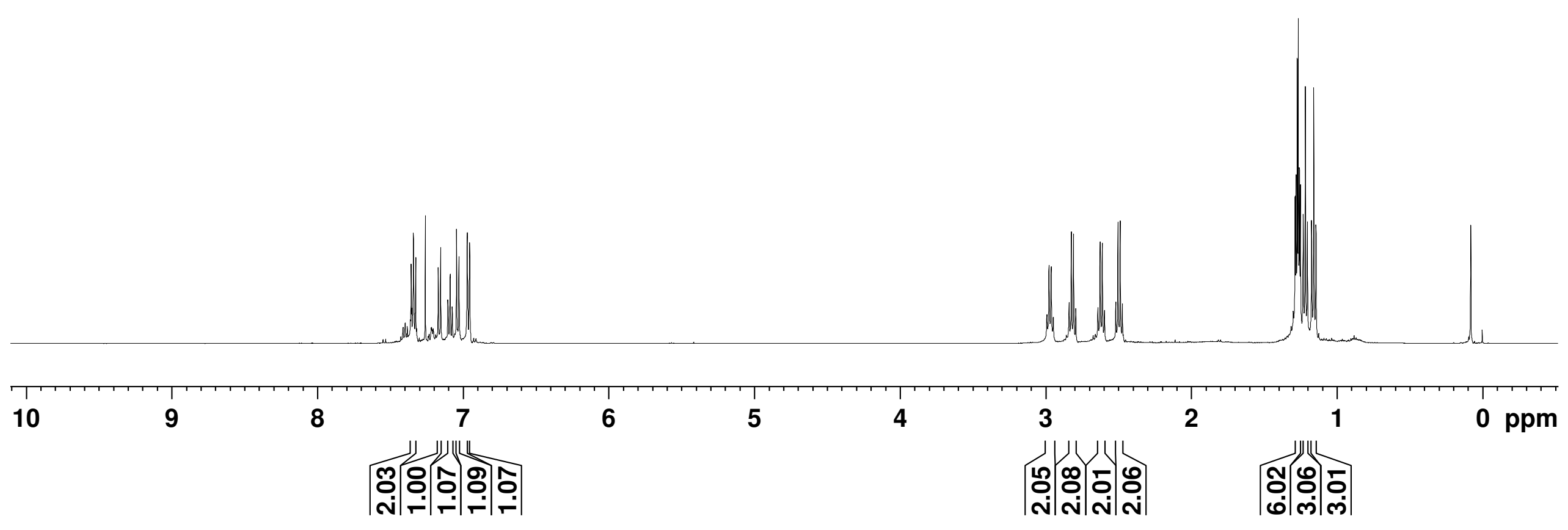




mj-126-15

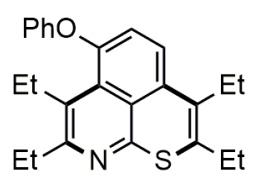

$3 \mathrm{~m}$, Scheme 1

${ }^{13} \mathrm{C}\left\{{ }^{1} \mathrm{H}\right\}$ NMR $\left(101 \mathrm{MHz}, \mathrm{CDCl}_{3}\right)$

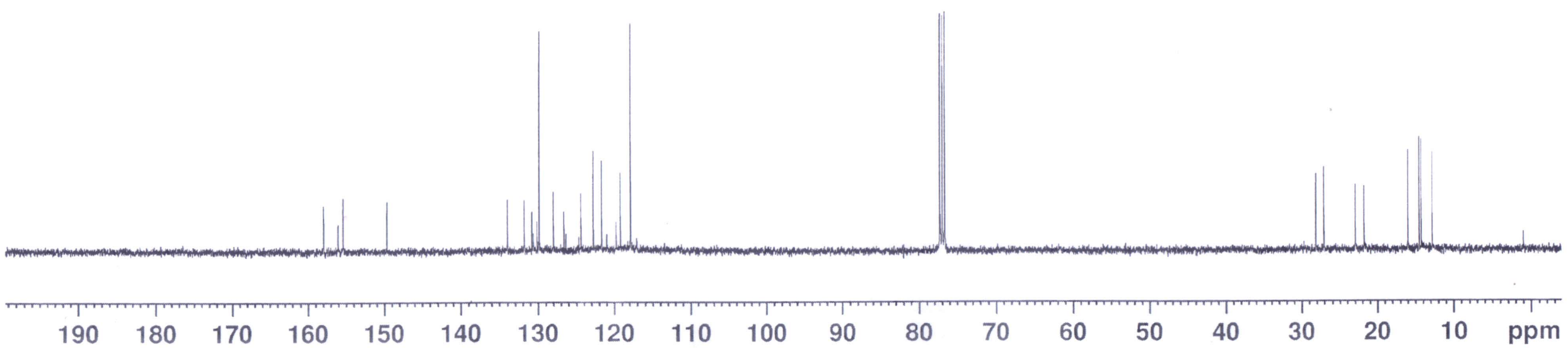


MJ-125-15

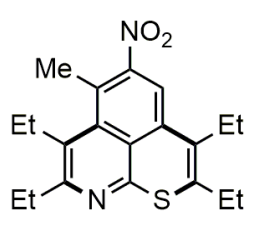

3n, Scheme 1

${ }^{1} \mathrm{H}$ NMR $\left(500 \mathrm{MHz}, \mathrm{CDCl}_{3}\right.$ ) 


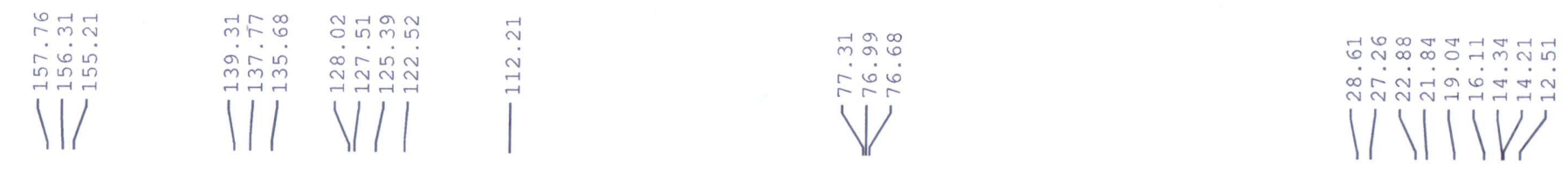

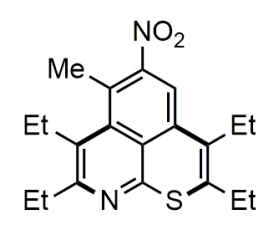

$m j-125-15$

3n, Scheme 1

${ }^{13} \mathrm{C}\left\{{ }^{1} \mathrm{H}\right\}$ NMR $\left(126 \mathrm{MHz}, \mathrm{CDCl}_{3}\right)$

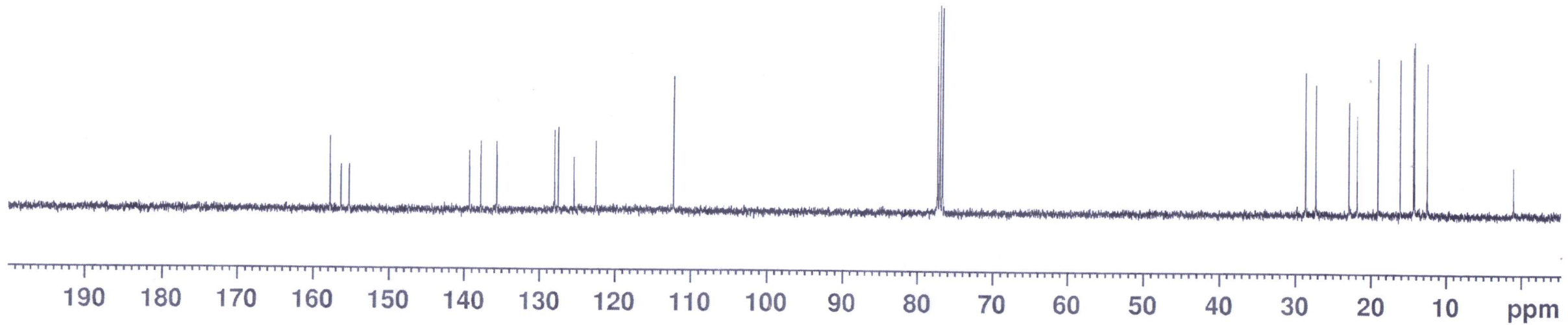




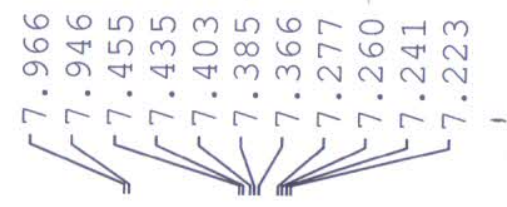

$m j-12-14$

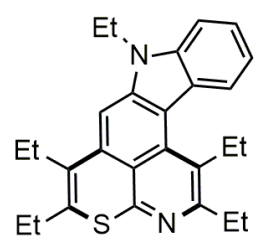

Bo, Scheme 1

${ }^{1} \mathrm{H}$ NMR $\left(400 \mathrm{MHz}, \mathrm{CDCl}_{3}\right)$

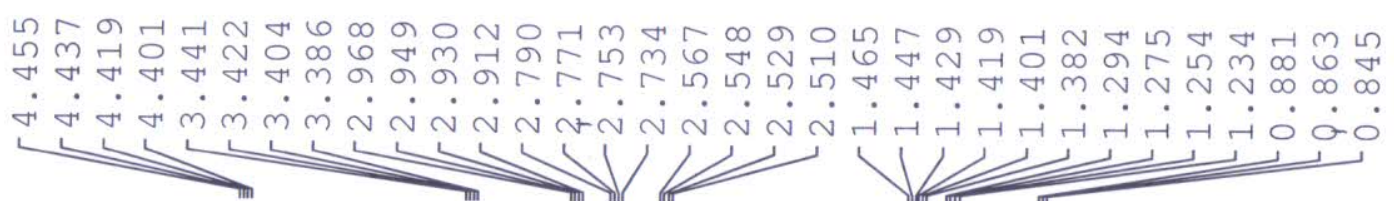

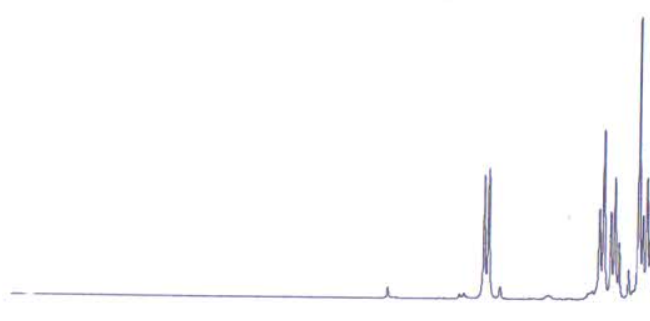

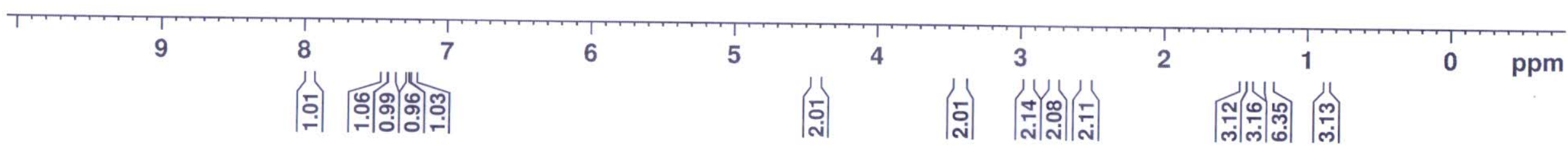

S 42 


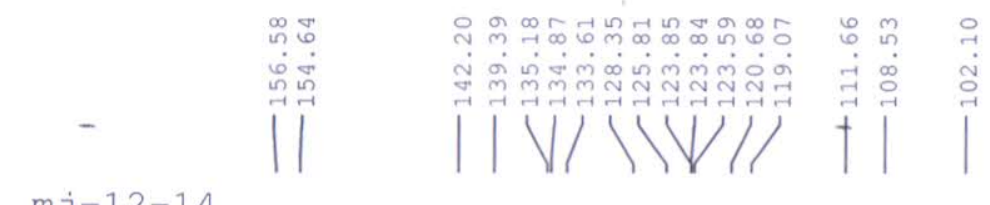

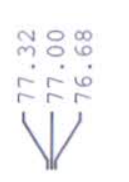

mj-12-14

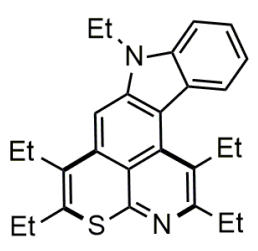

3o, Scheme 1

${ }^{13} \mathrm{C}\left\{{ }^{1} \mathrm{H}\right\}$ NMR $\left(101 \mathrm{MHz}, \mathrm{CDCl}_{3}\right)$

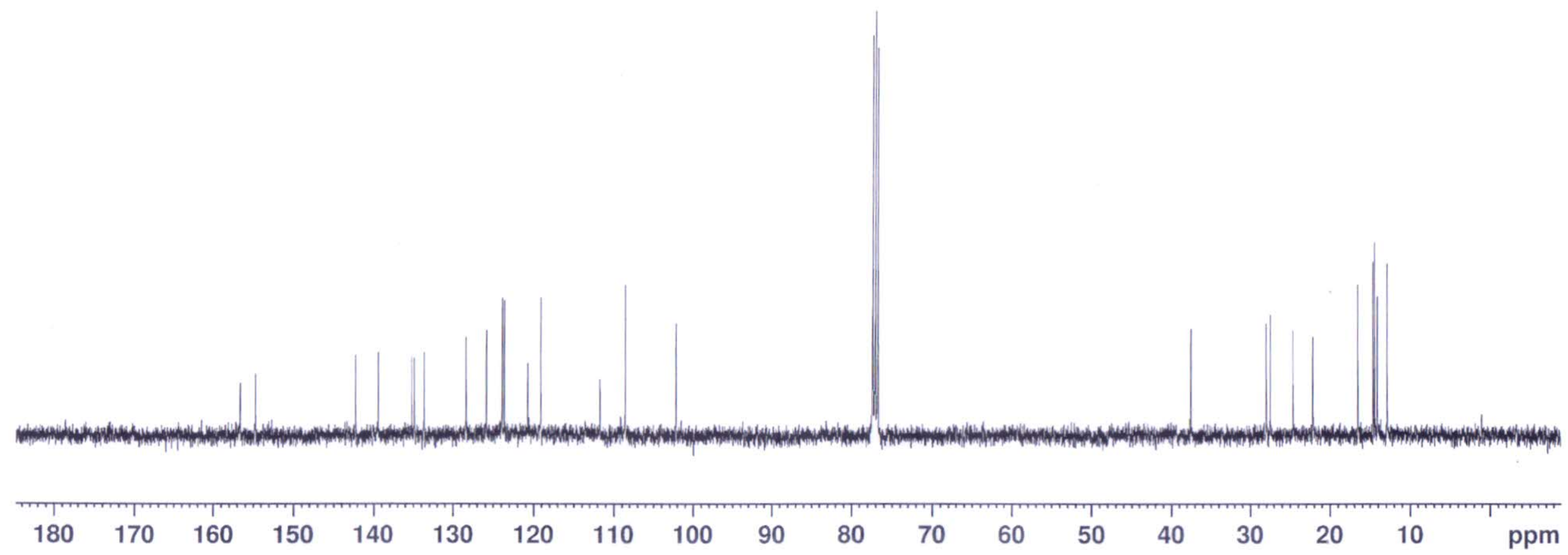




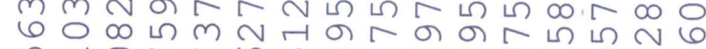

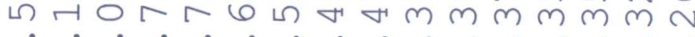
momaririririririr

年,

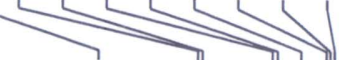

H

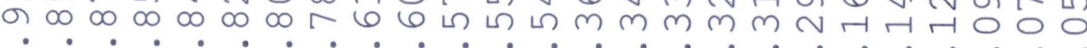

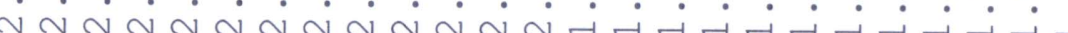

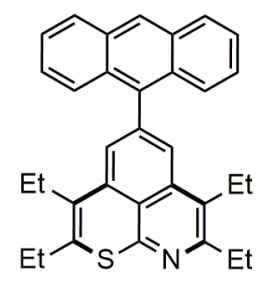

3p, Scheme 1

${ }^{1} \mathrm{H} \mathrm{NMR}\left(400 \mathrm{MHz}, \mathrm{CDCl}_{3}\right)$

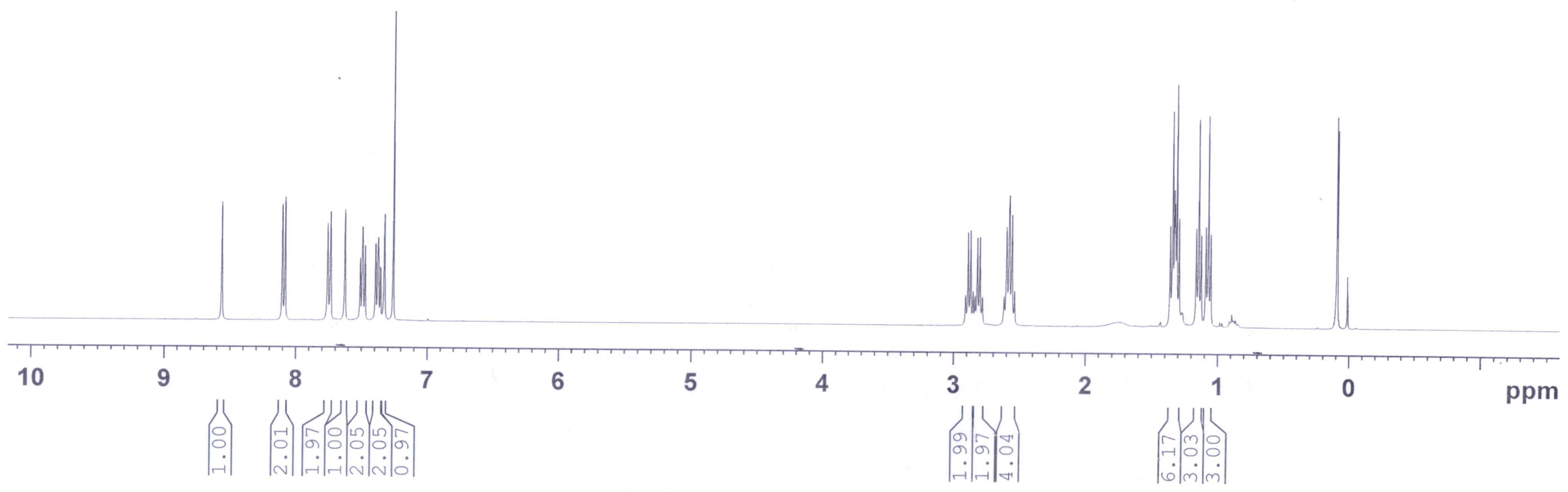



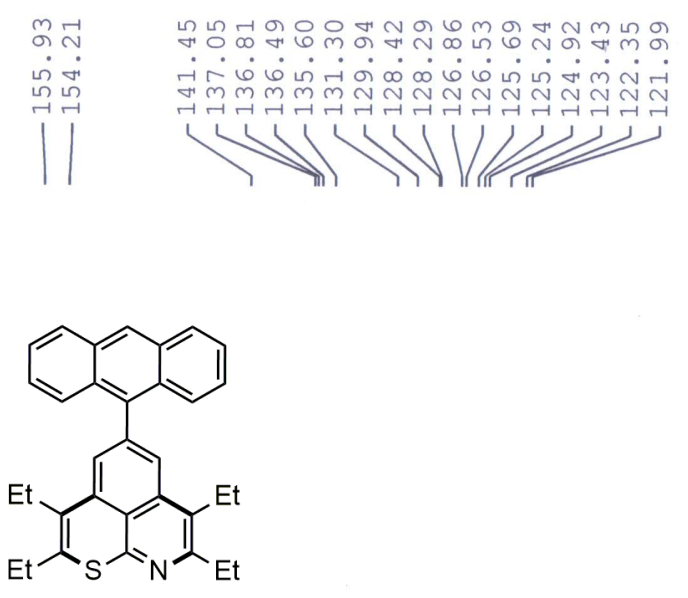

$3 p$, Scheme 1

${ }^{13} \mathrm{C}\left\{{ }^{1} \mathrm{H}\right\}$ NMR $\left(101 \mathrm{MHz}, \mathrm{CDCl}_{3}\right)$

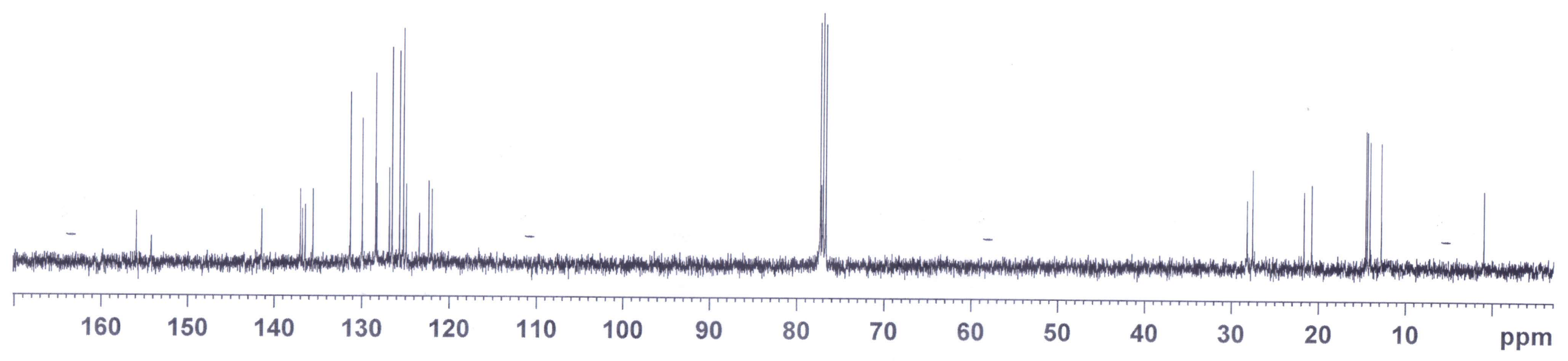


SRS-1-29

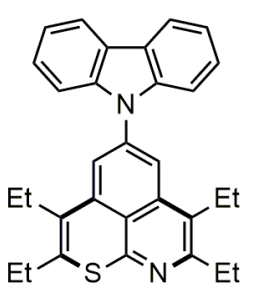

3q, Scheme 1

${ }^{1} \mathrm{H} \mathrm{NMR}\left(500 \mathrm{MHz}, \mathrm{CDCl}_{3}\right)$

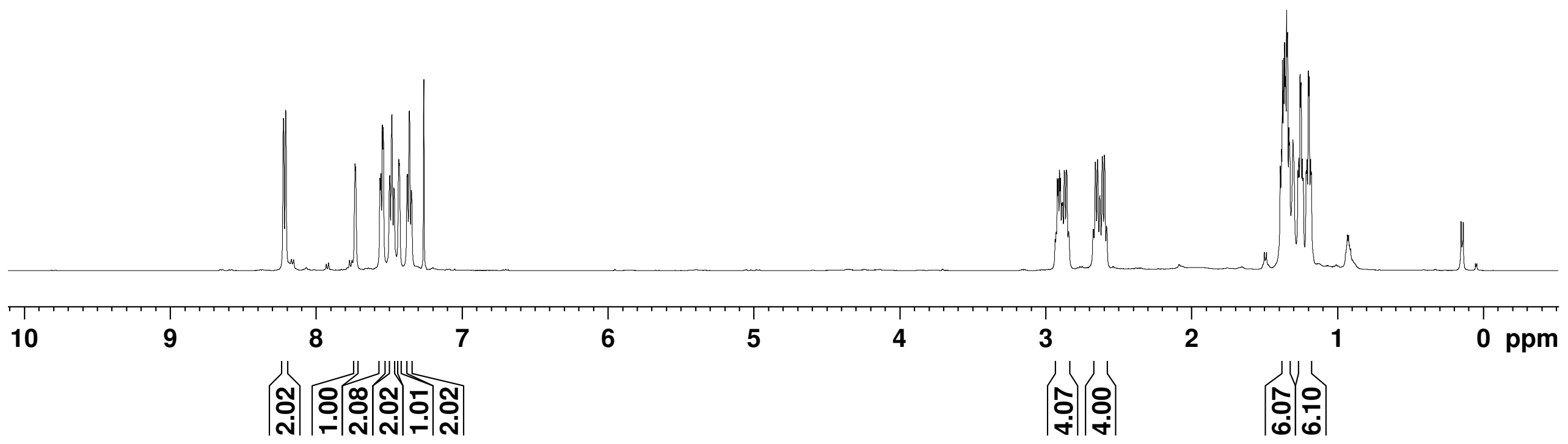




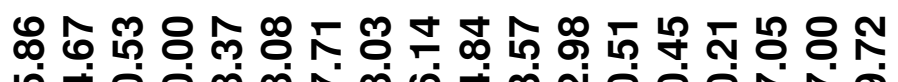

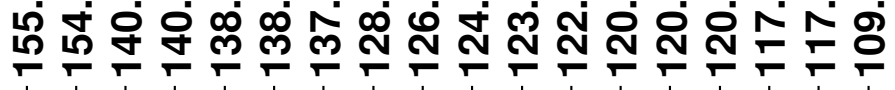

ᄂ

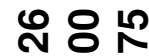

กペ

V

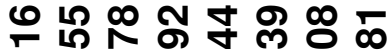

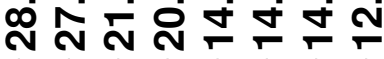

SRS-1-29

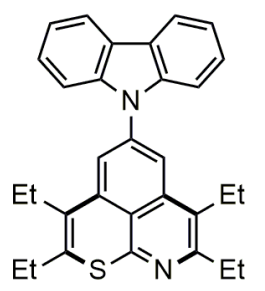

3q, Scheme 1

${ }^{13} \mathrm{C}\left\{{ }^{1} \mathrm{H}\right\}$ NMR $\left(126 \mathrm{MHz}, \mathrm{CDCl}_{3}\right)$

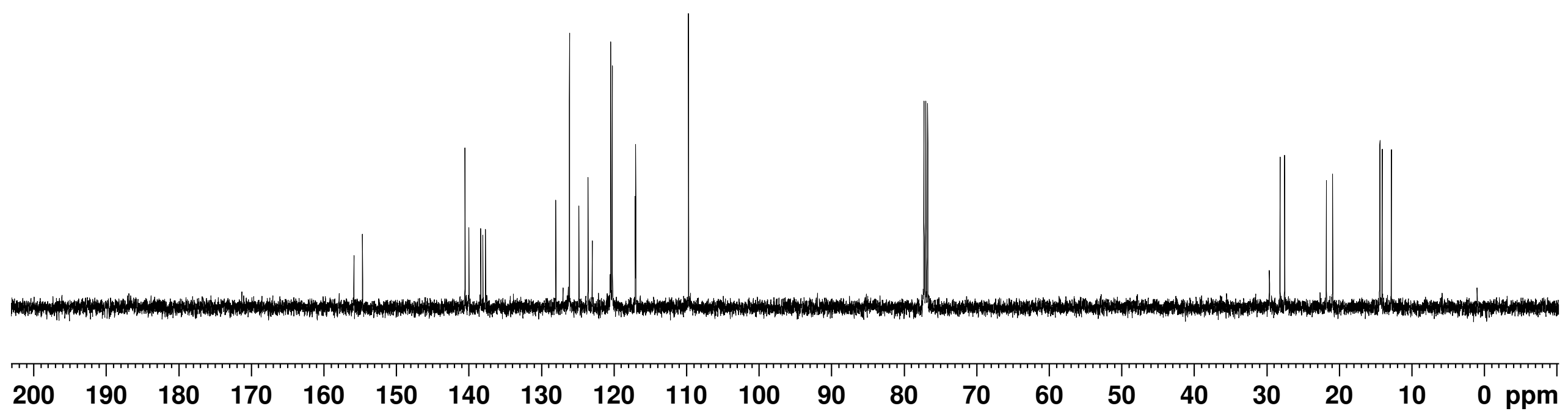




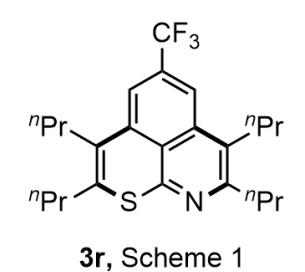

$1 \mathrm{H} \mathrm{NMR}\left(500 \mathrm{MHz}, \mathrm{CDCl}_{3}\right)$

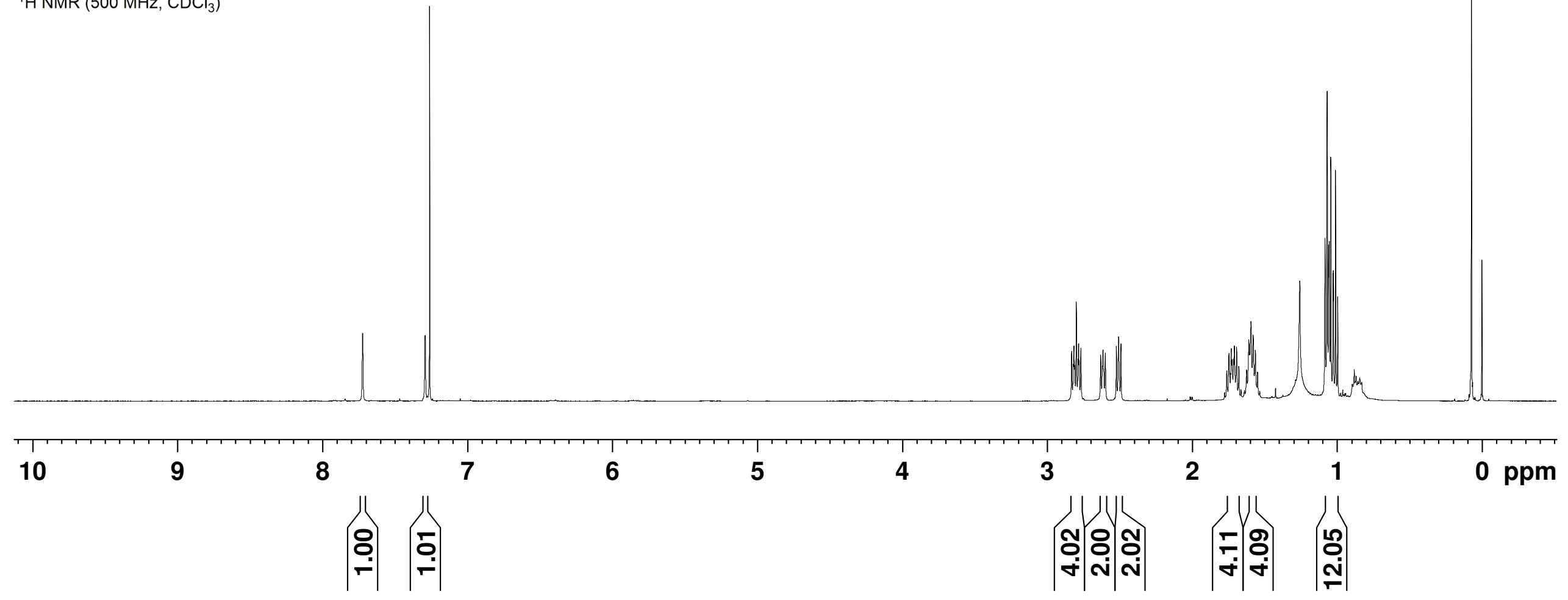


SRS-1-96-3

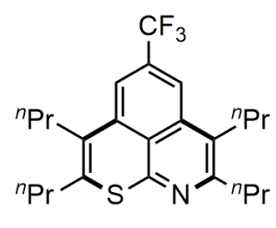

$3 r$, Scheme 1

${ }^{13} \mathrm{C}\left\{{ }^{1} \mathrm{H}\right\}$ NMR $\left(126 \mathrm{MHz}, \mathrm{CDCl}_{3}\right)$

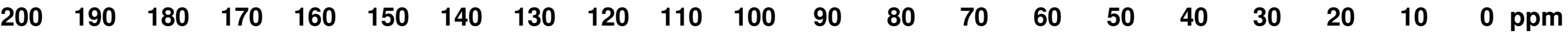




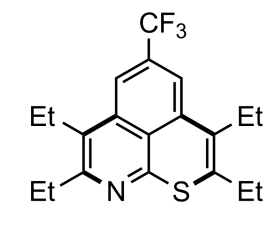

3r, Scheme 1

${ }^{19} \mathrm{~F} \mathrm{NMR} \mathrm{(376} \mathrm{MHz,} \mathrm{CDCl}_{3}$ )

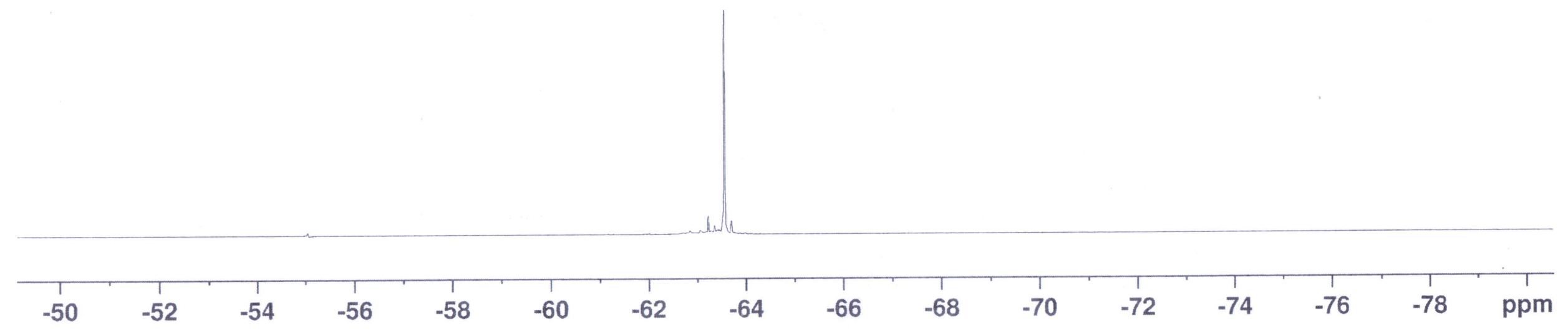


AS-18

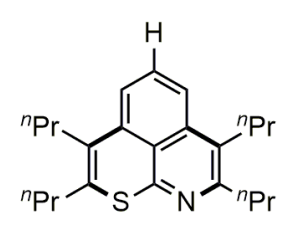

3s, Scheme 1

${ }^{1} \mathrm{HNMR}\left(500 \mathrm{MHz}, \mathrm{CDCl}_{3}\right)$

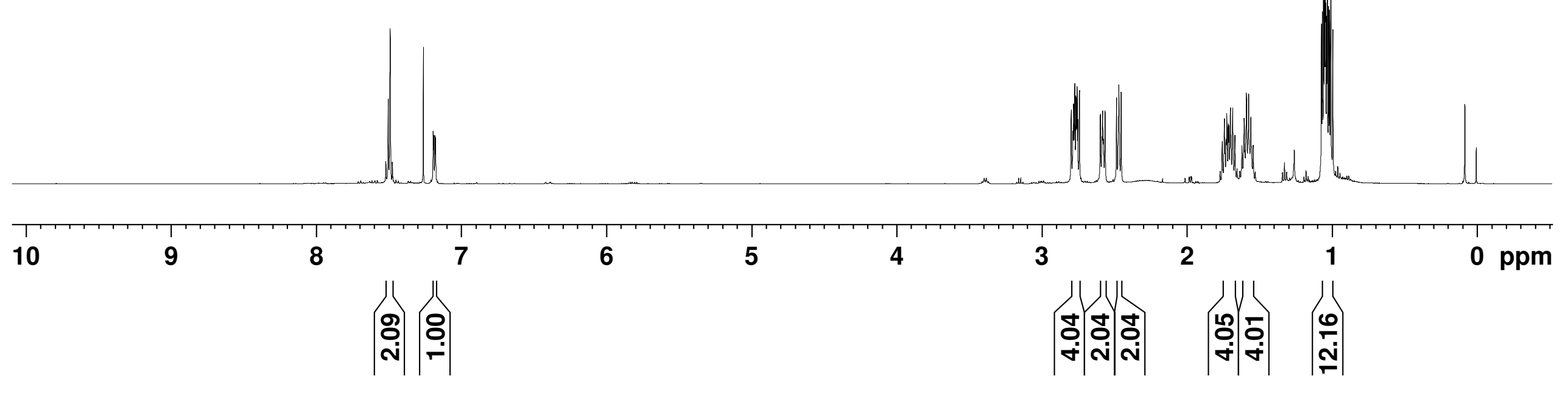




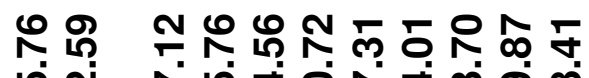

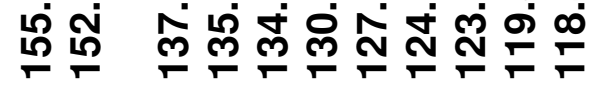

广

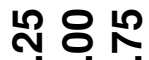

ヘト゚

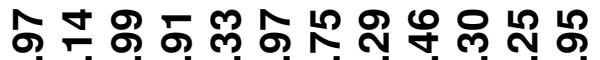

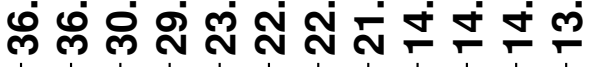

AS-18

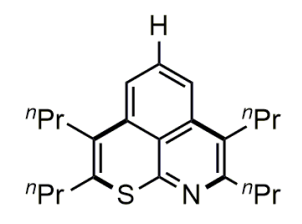

3s, Scheme 1

${ }^{13} \mathrm{C}\left\{{ }^{1} \mathrm{H}\right\} \mathrm{NMR}\left(101 \mathrm{MHz}, \mathrm{CDCl}_{3}\right)$

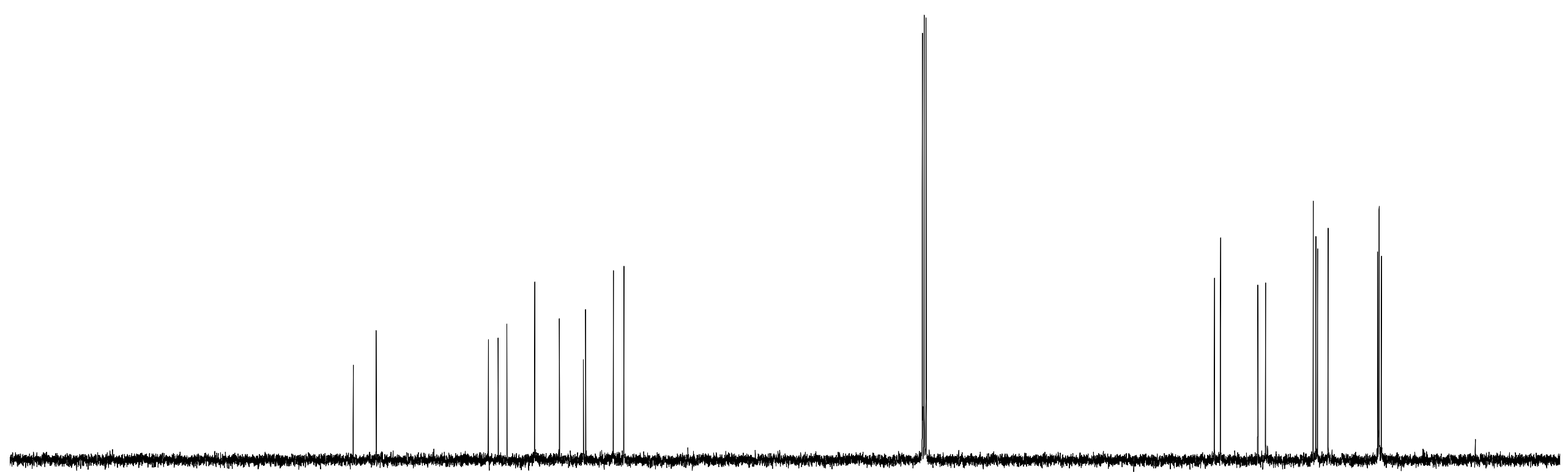




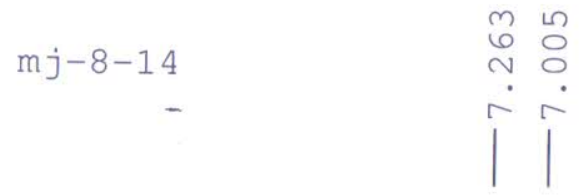

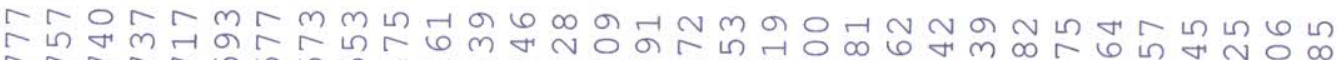

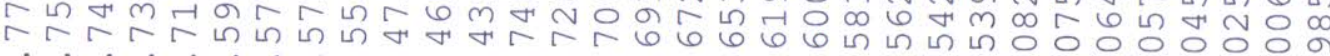

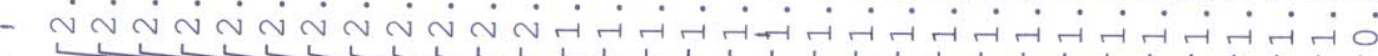

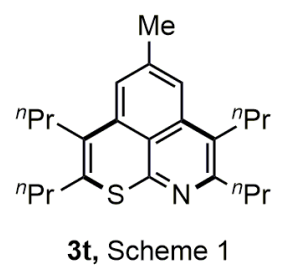

${ }^{1} \mathrm{H}$ NMR $\left(400 \mathrm{MHz}, \mathrm{CDCl}_{3}\right)$

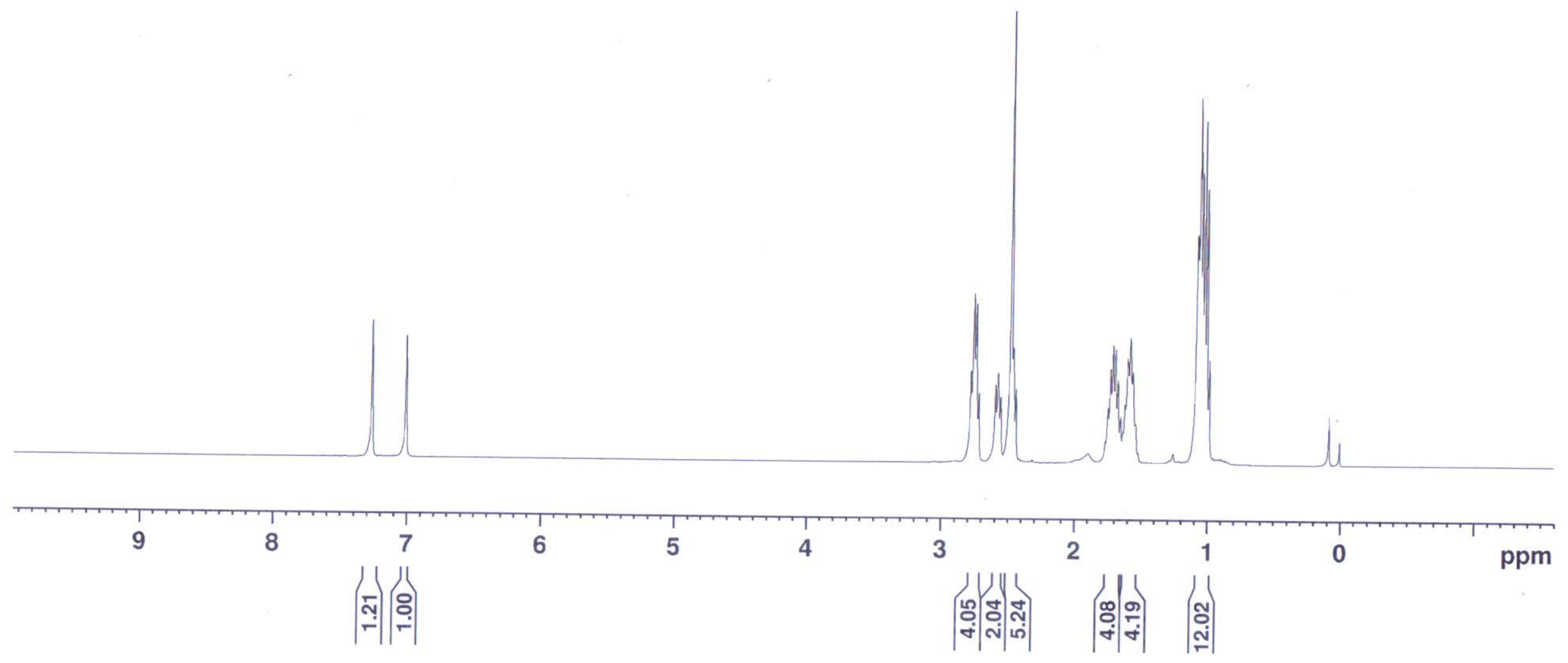



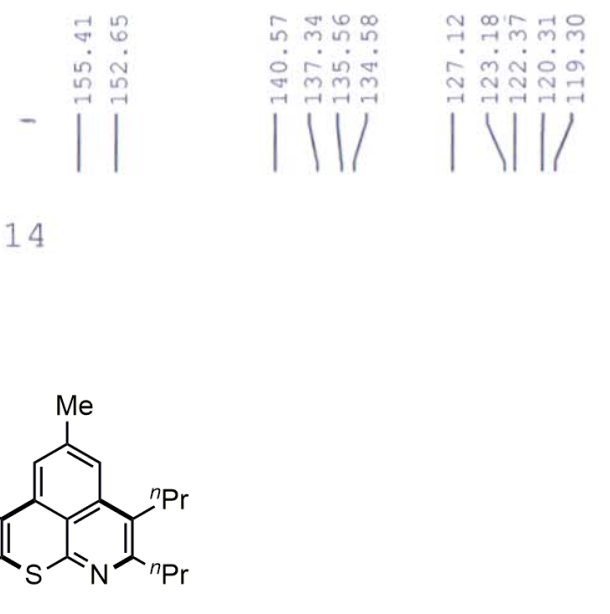

$m j-8-14$

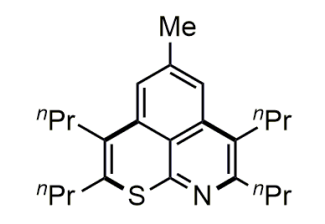

3t, Scheme 1

${ }^{13} \mathrm{C}\left\{{ }^{1} \mathrm{H}\right\}$ NMR $\left(101 \mathrm{MHz}, \mathrm{CDCl}_{3}\right)$

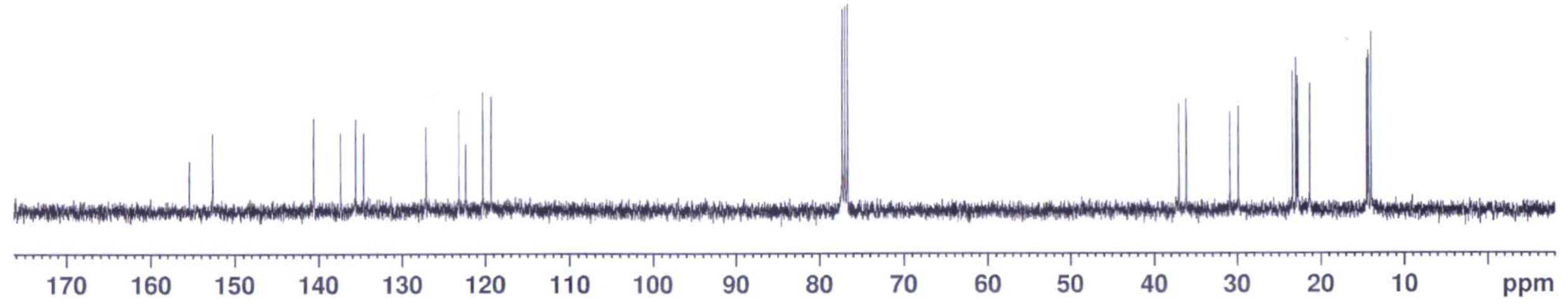


$m j-7-14$

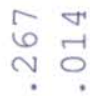

i

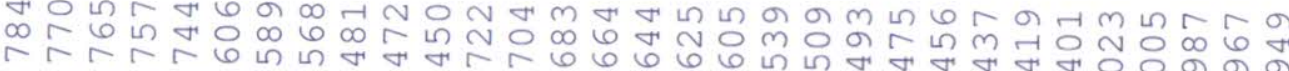

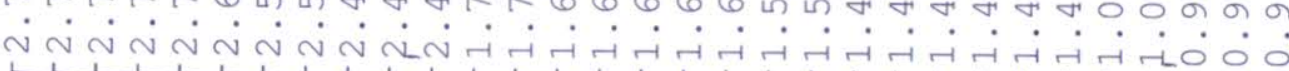

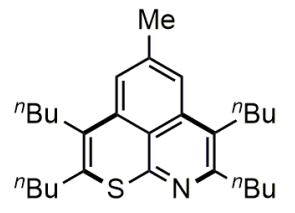

3u, Scheme 1

${ }^{1} \mathrm{H}$ NMR (400 MHz, $\mathrm{CDCl}_{3}$ )

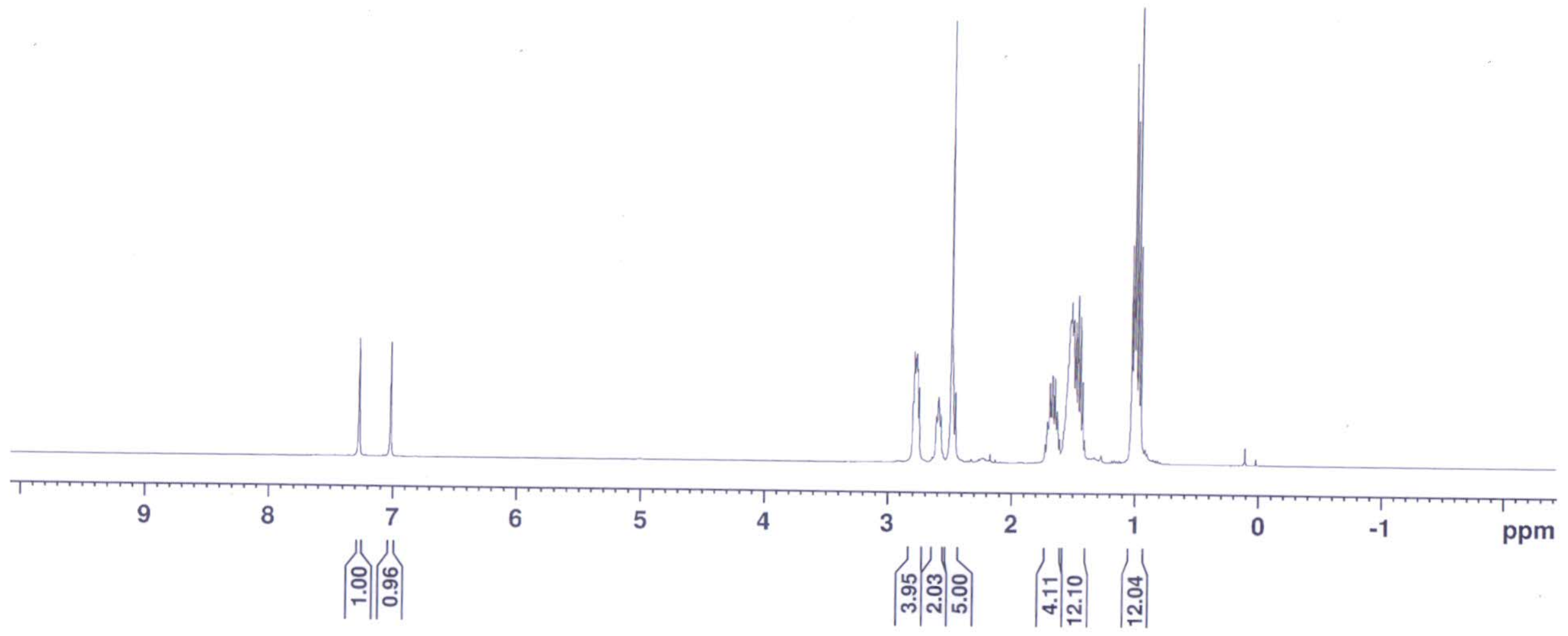



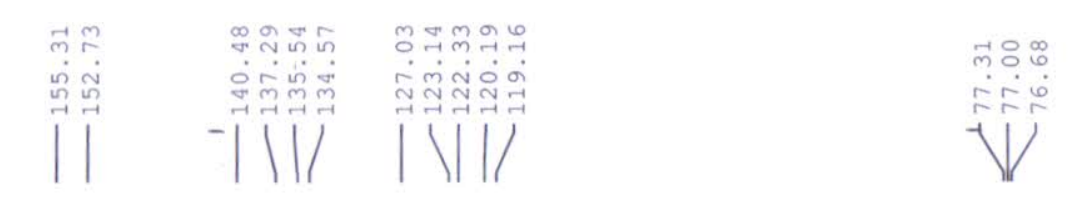

$m j-7-14$

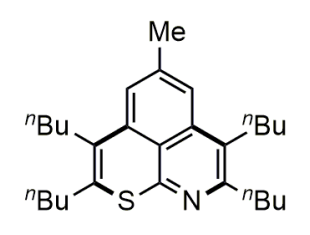

3u, Scheme 1

${ }^{13} \mathrm{C}\left\{{ }^{1} \mathrm{H}\right\}$ NMR $\left(101 \mathrm{MHz}, \mathrm{CDCl}_{3}\right)$
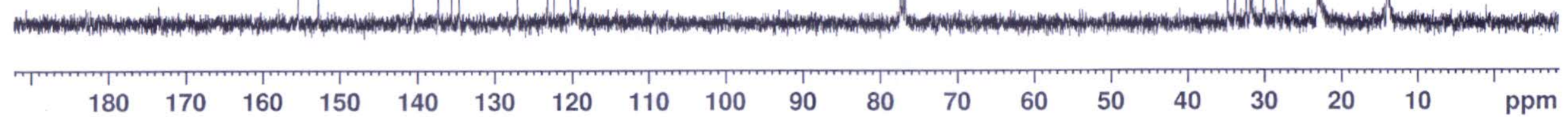
$\mathrm{MJ}-128-15$

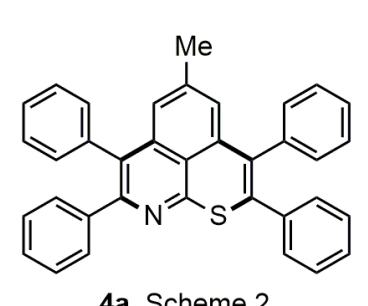

4a, Scheme 2

${ }^{1} \mathrm{H} \mathrm{NMR}\left(500 \mathrm{MHz}, \mathrm{CDCl}_{3}\right)$

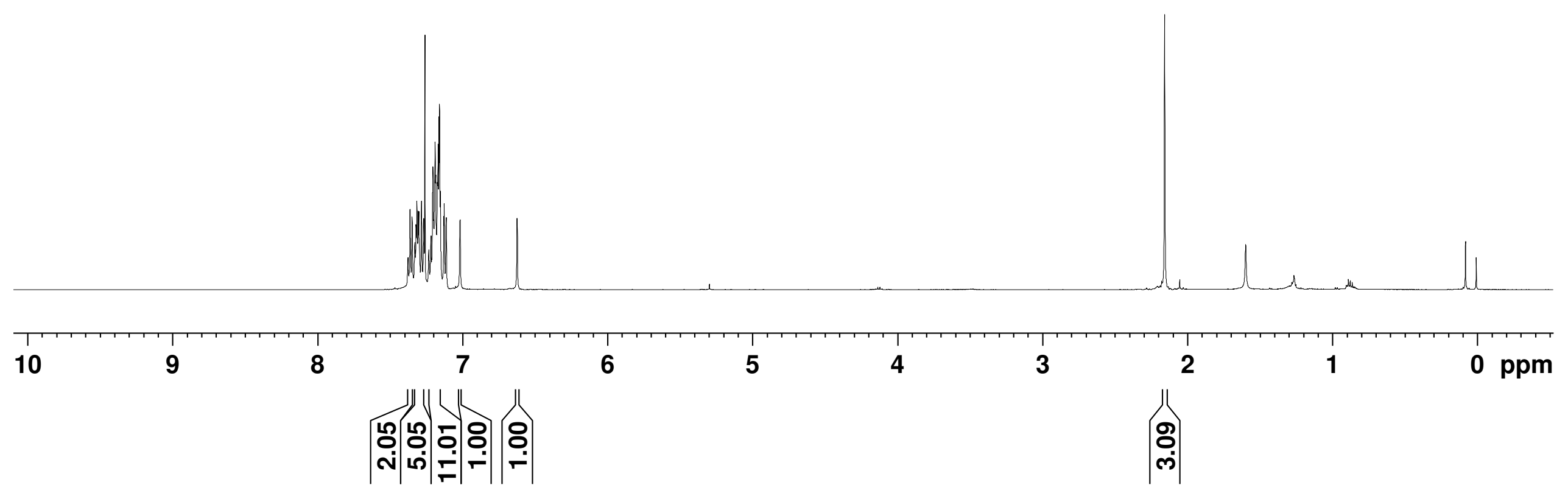




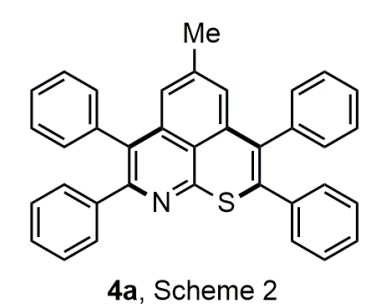

${ }^{13} \mathrm{C}\left\{{ }^{1} \mathrm{H}\right\} \mathrm{NMR}\left(101 \mathrm{MHz}, \mathrm{CDCl}_{3}\right)$

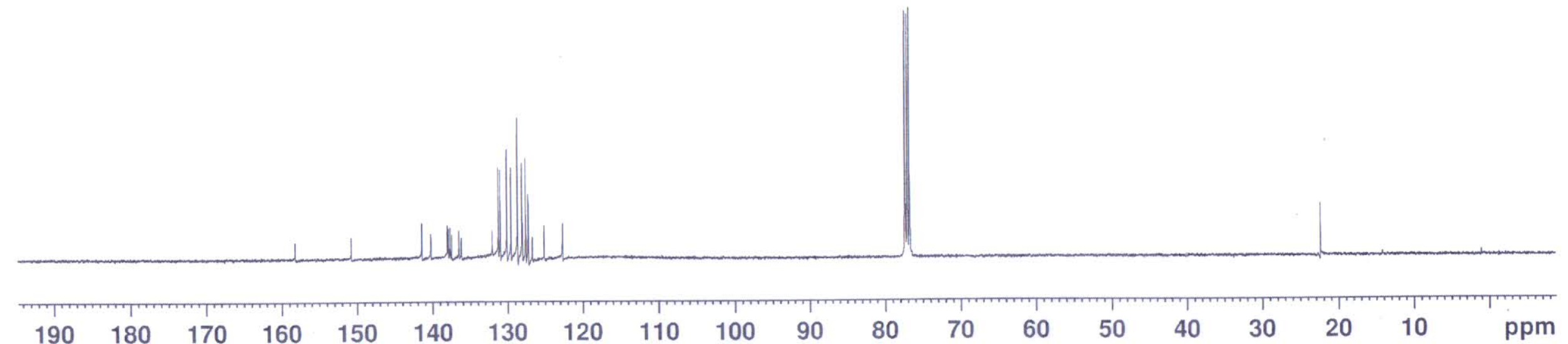




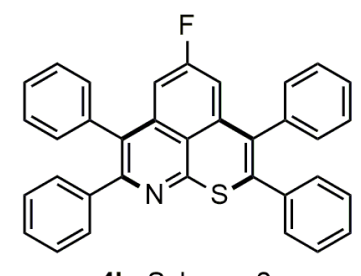

4b, Scheme 2

${ }^{1} \mathrm{H}$ NMR $\left(400 \mathrm{MHz}, \mathrm{CDCl}_{3}\right)$

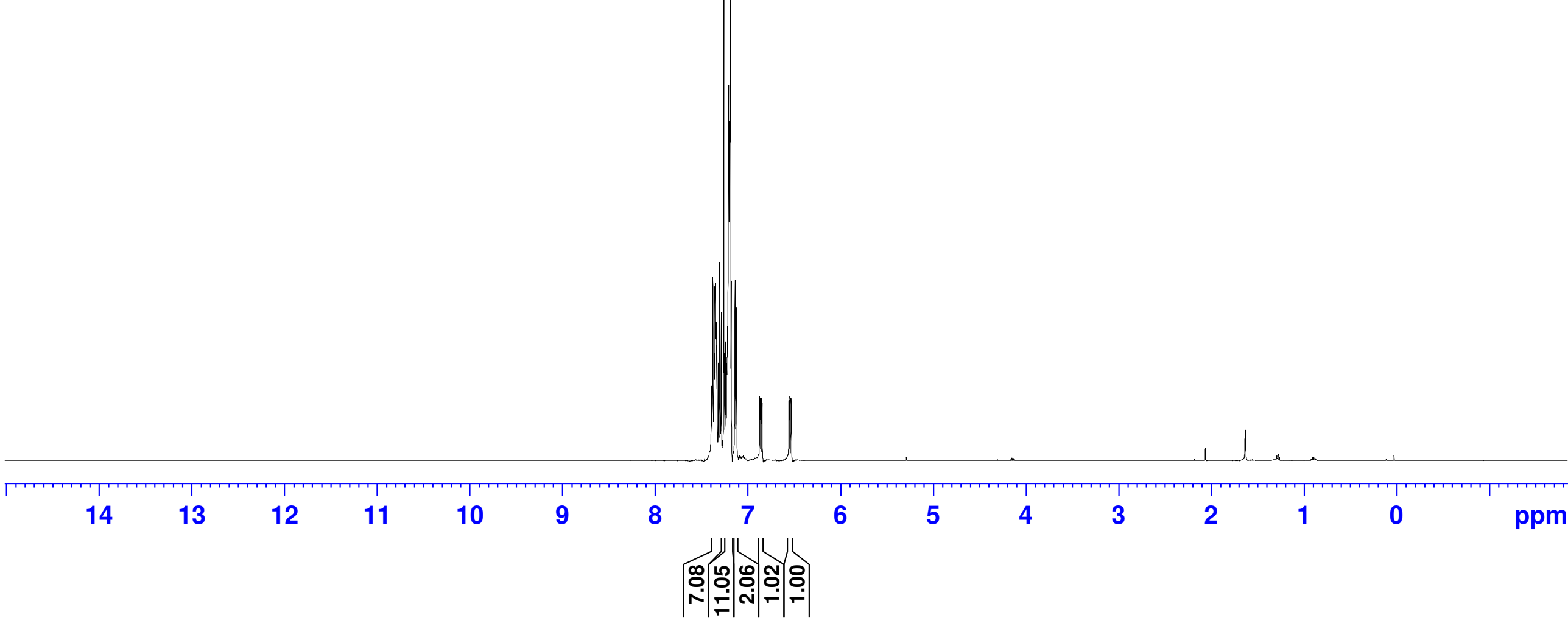




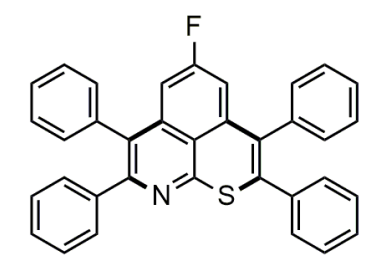

4b, Scheme 2

${ }^{13} \mathrm{C}\left\{{ }^{1} \mathrm{H}\right\} \mathrm{NMR}\left(101 \mathrm{MHz}, \mathrm{CDCl}_{3}\right)$
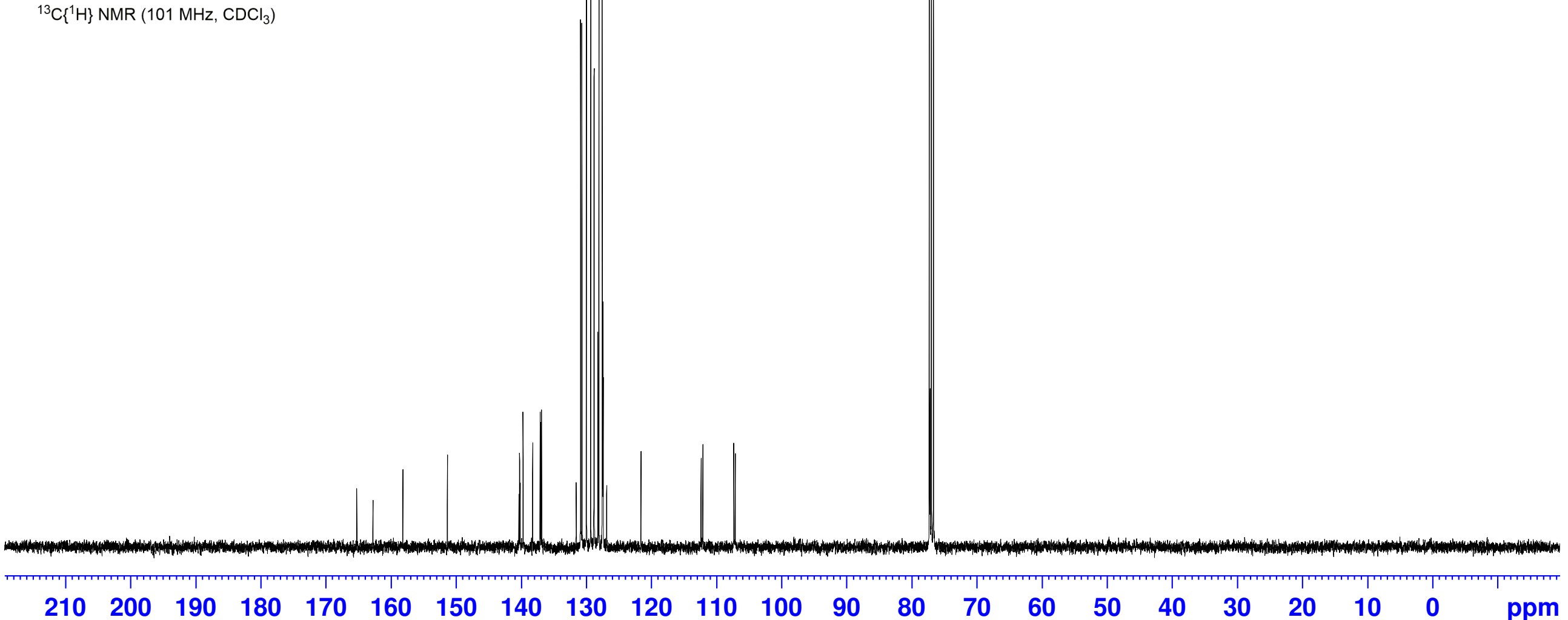


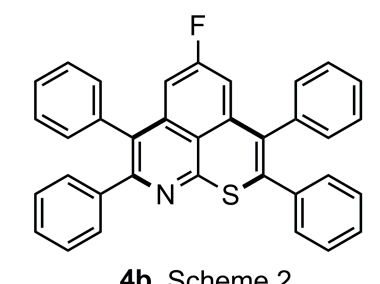

4b, Scheme 2

${ }^{19} \mathrm{~F} \mathrm{NMR}\left(376 \mathrm{MHz}, \mathrm{CDCl}_{3}\right)$

\begin{tabular}{|lllllllllll|ll|}
\hline 0 & -20 & -40 & -60 & -80 & -100 & -120 & -140 & -160 & -180 & -200 & ppm
\end{tabular}




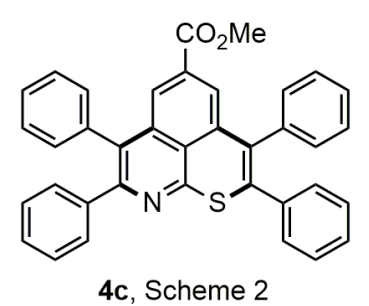

4c, Scheme 2

${ }^{1} \mathrm{H} \mathrm{NMR}\left(500 \mathrm{MHz}, \mathrm{CDCl}_{3}\right.$ )

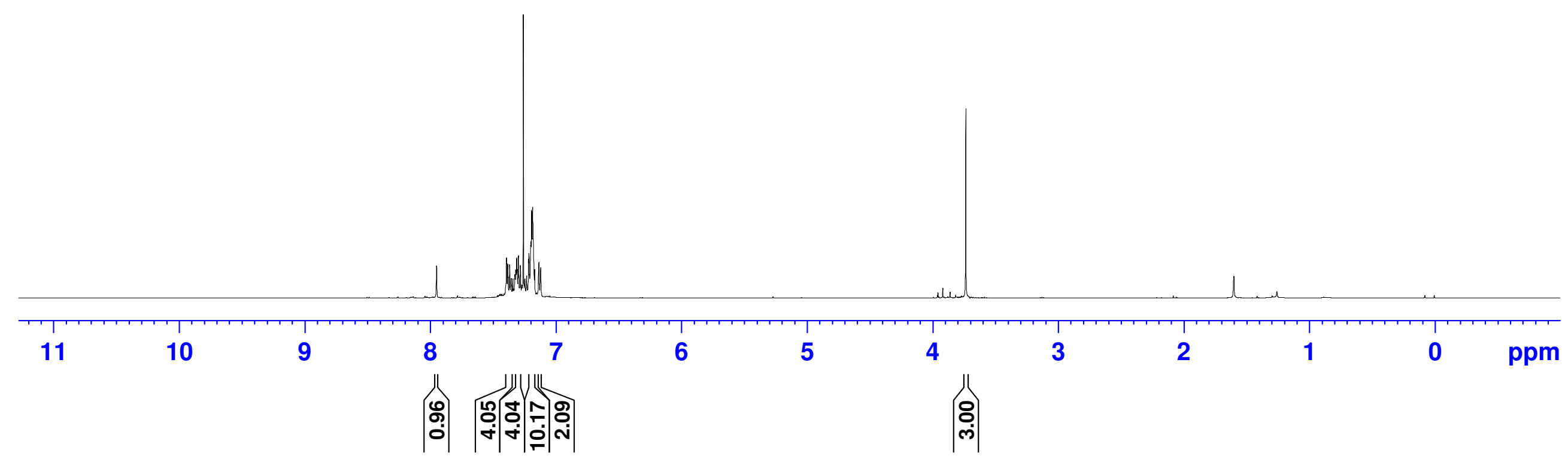


Ag $-3-113$

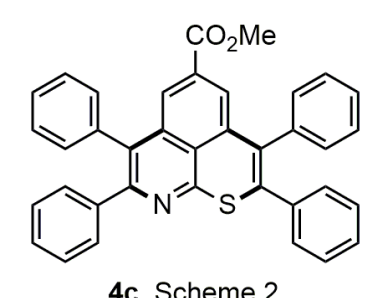

4c, Scheme 2

${ }^{13} \mathrm{C}\left\{{ }^{1} \mathrm{H}\right\} \mathrm{NMR}\left(101 \mathrm{MHz}, \mathrm{CDCl}_{3}\right)$
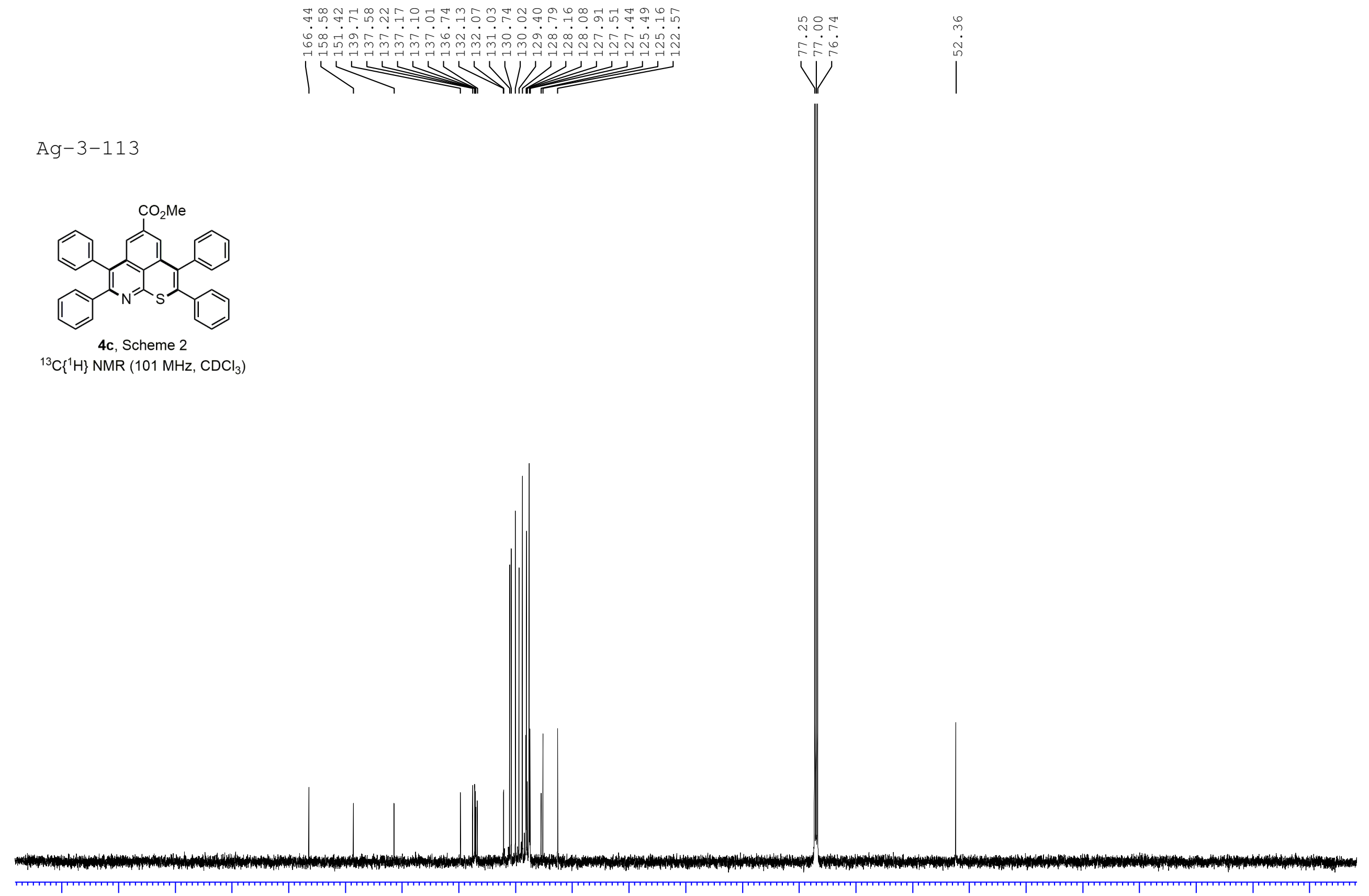

$\begin{array}{lllllllllllllllllllllllll}210 & 200 & 190 & 180 & 170 & 160 & 150 & 140 & 130 & 120 & 110 & 100 & 90 & 80 & 70 & 60 & 50 & 40 & 30 & 20 & 10 & 0 & \text { ppm }\end{array}$ 
ARIJIT -AS-108

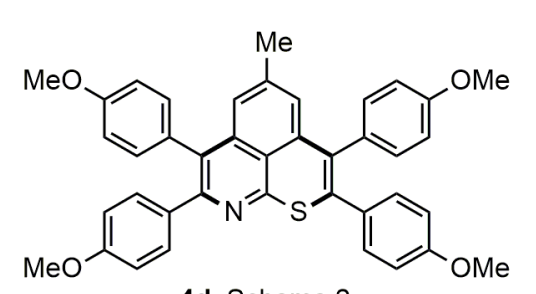

4d Scheme 2

${ }^{1} \mathrm{H} \mathrm{NMR}\left(400 \mathrm{MHz}, \mathrm{CDCl}_{3}\right)$
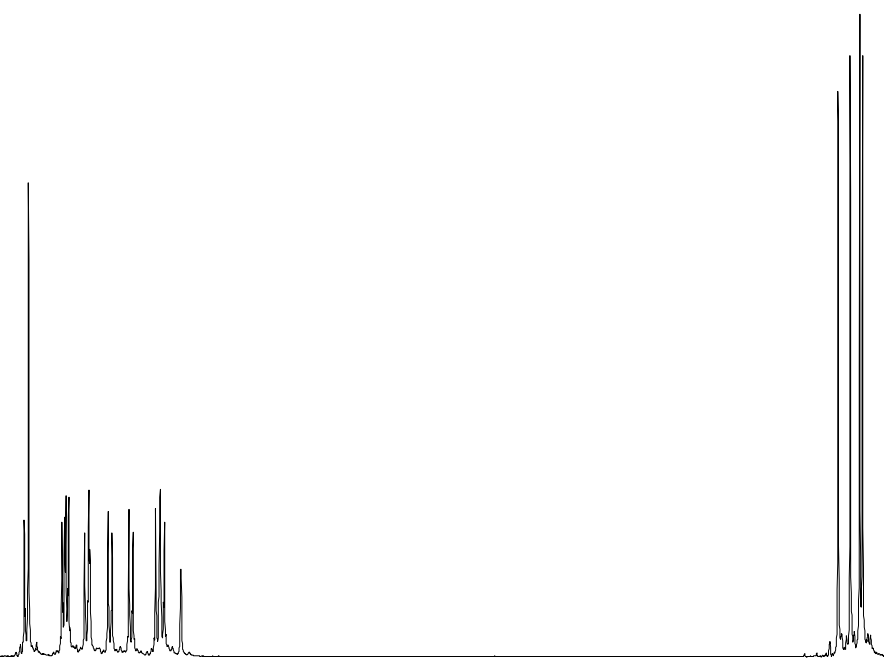

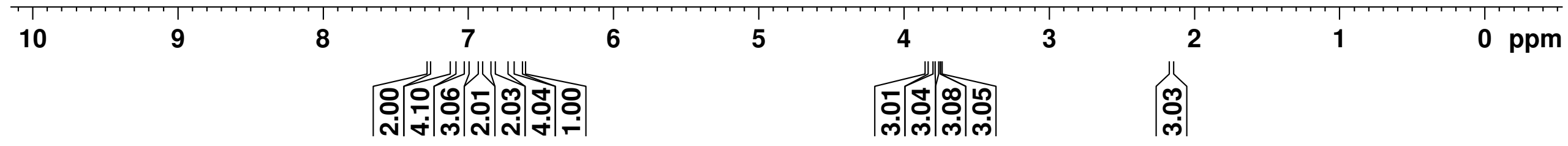


ARIJIT -AS-108

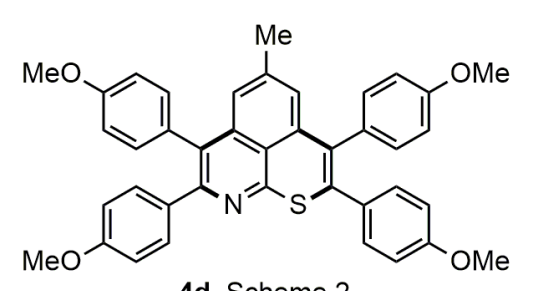

4d, Scheme 2

${ }^{13} \mathrm{C}\left\{{ }^{1} \mathrm{H}\right\}$ NMR $\left(101 \mathrm{MHz}, \mathrm{CDCl}_{3}\right)$

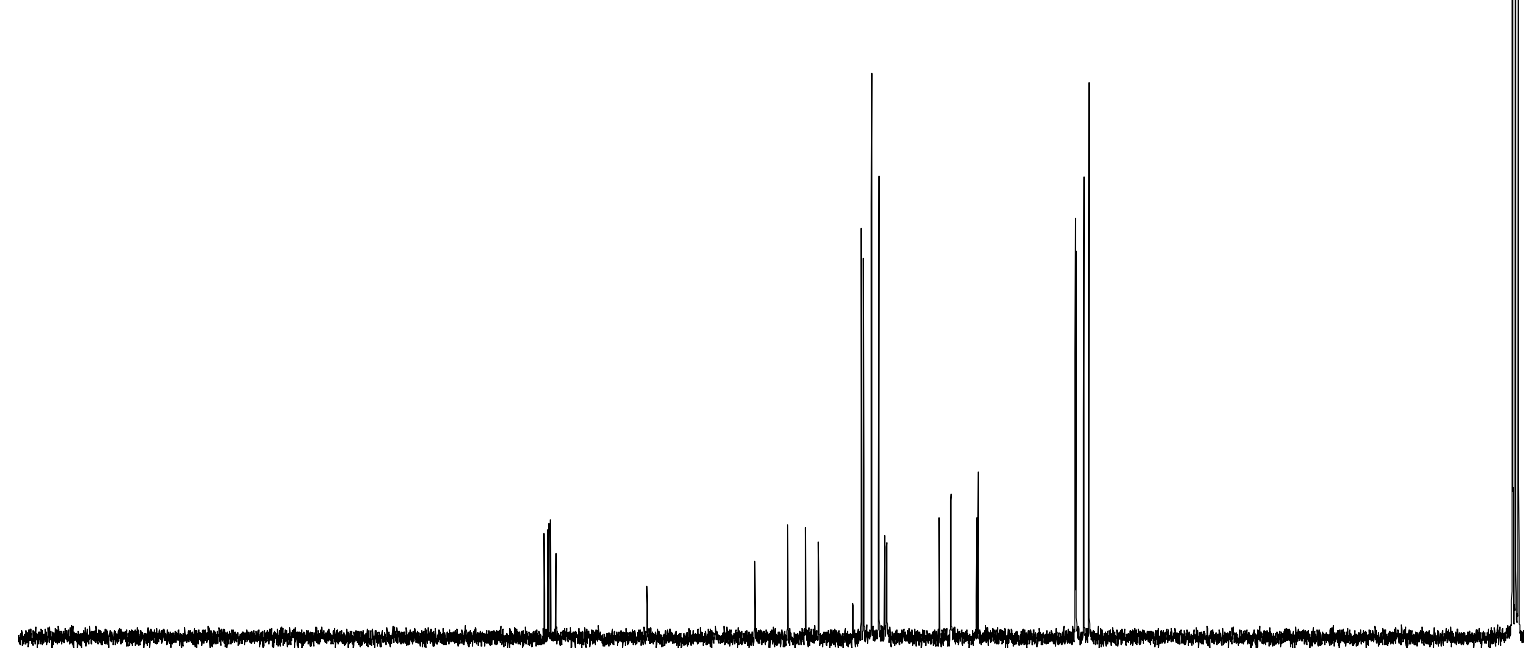

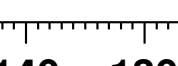
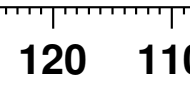

110100

90
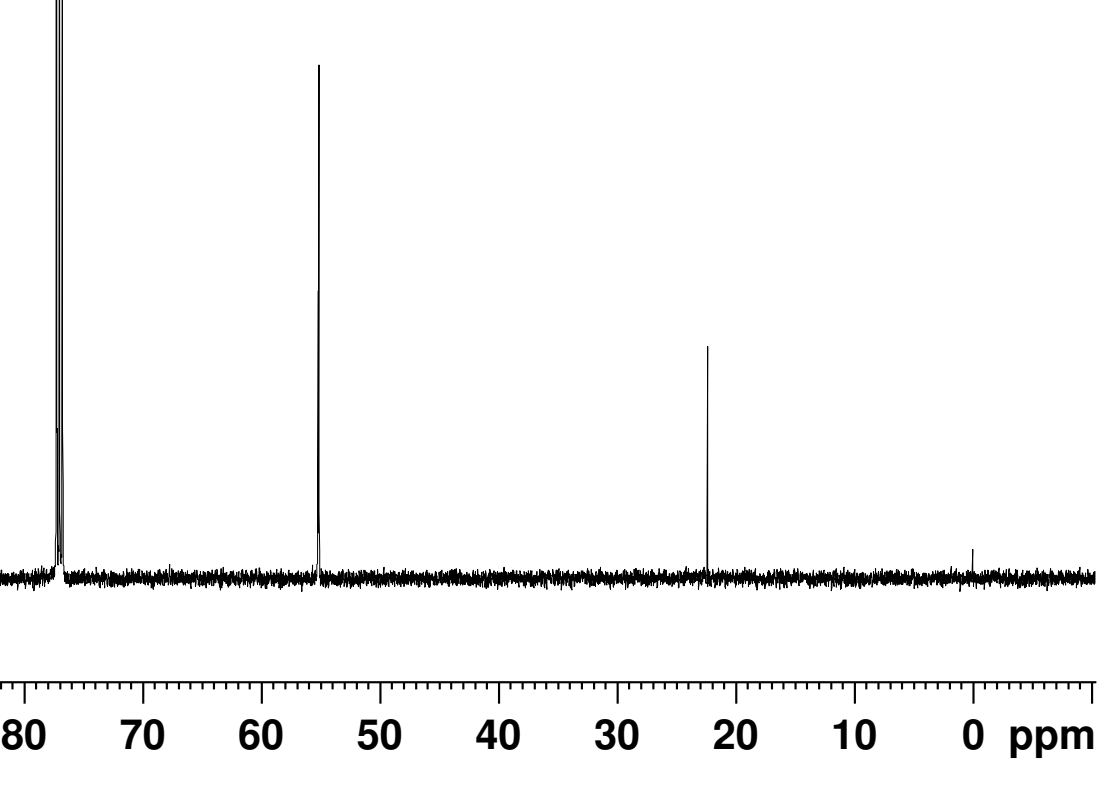


\section{ARIJIT -AS-107}
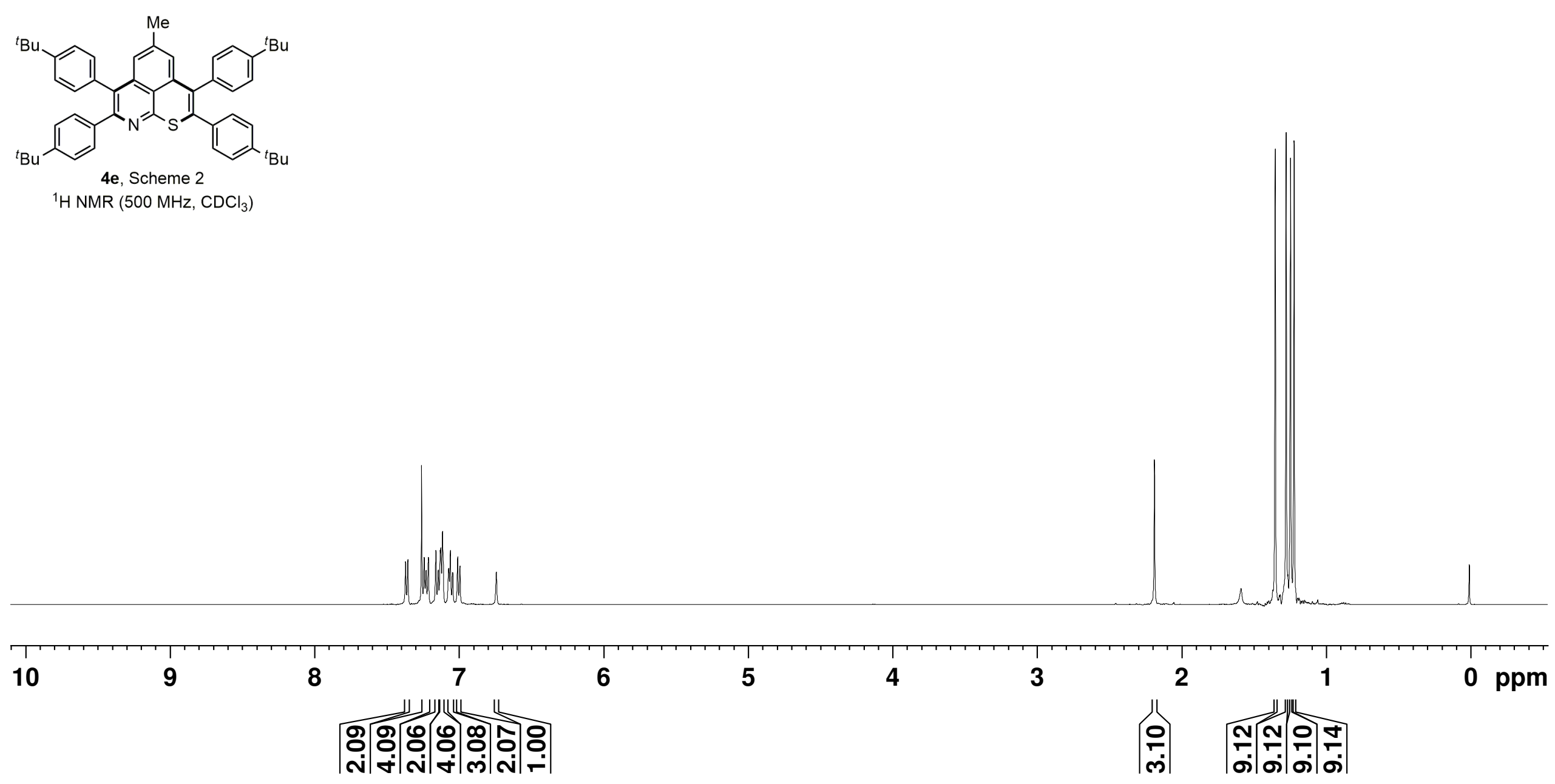


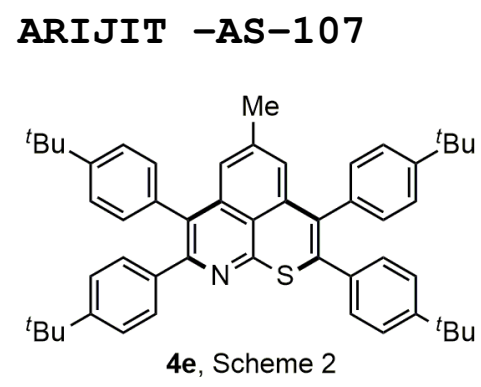

${ }^{13} \mathrm{C}\left\{{ }^{1} \mathrm{H}\right\}$ NMR $\left(101 \mathrm{MHz}, \mathrm{CDCl}_{3}\right)$

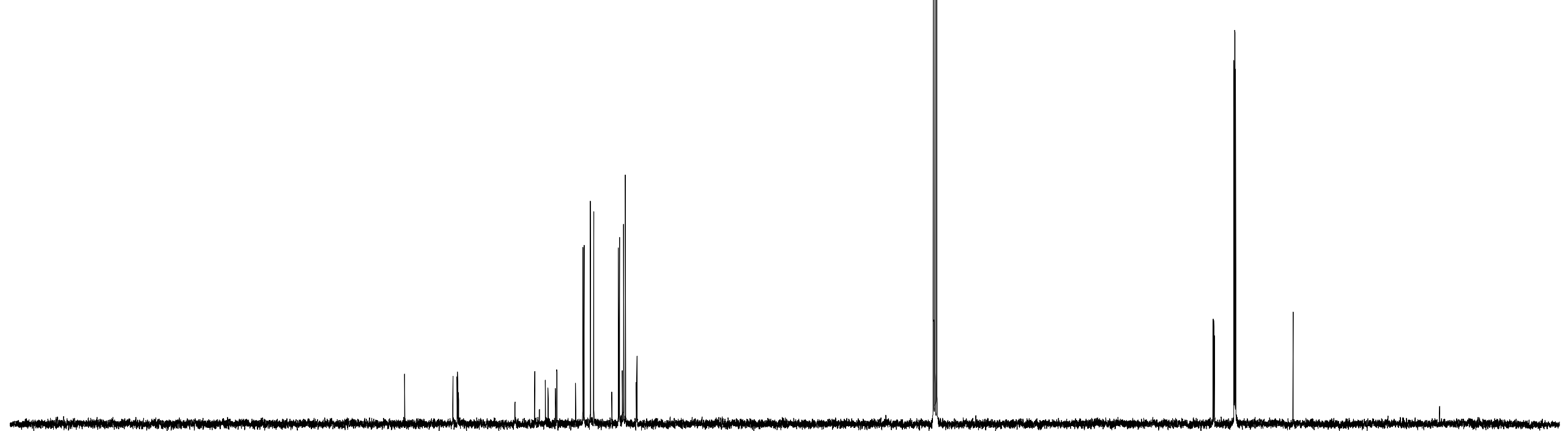

$\begin{array}{llllllllllllllllllllllll}210 & 200 & 190 & 180 & 170 & 160 & 150 & 140 & 130 & 120 & 110 & 100 & 90 & 80 & 70 & 60 & 50 & 40 & 30 & 20 & 10 & 0 & \text { ppm }\end{array}$ 


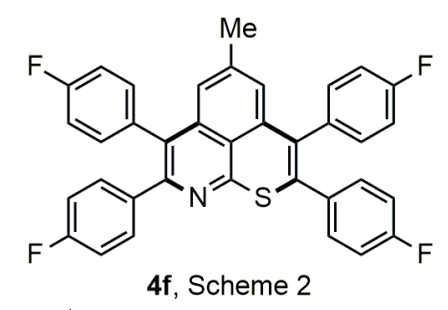

${ }^{1} \mathrm{HNMR}\left(400 \mathrm{MHz}, \mathrm{CDCl}_{3}\right)$
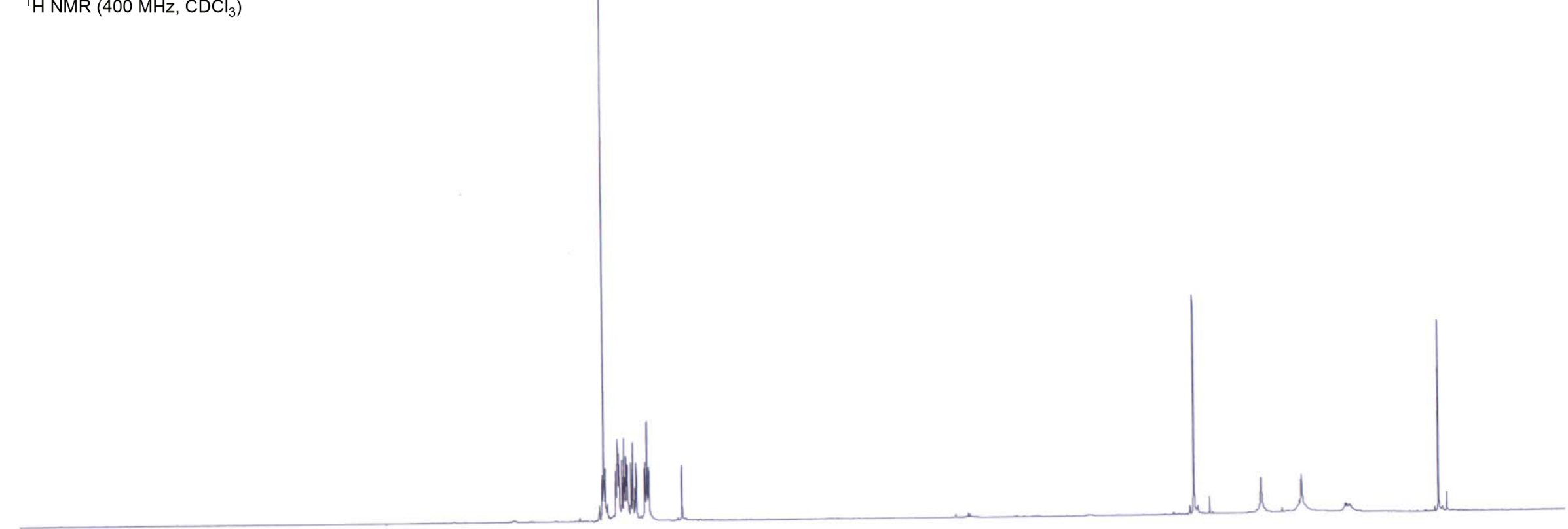

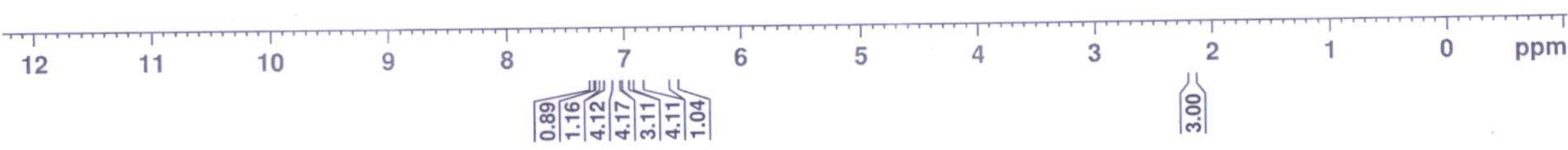




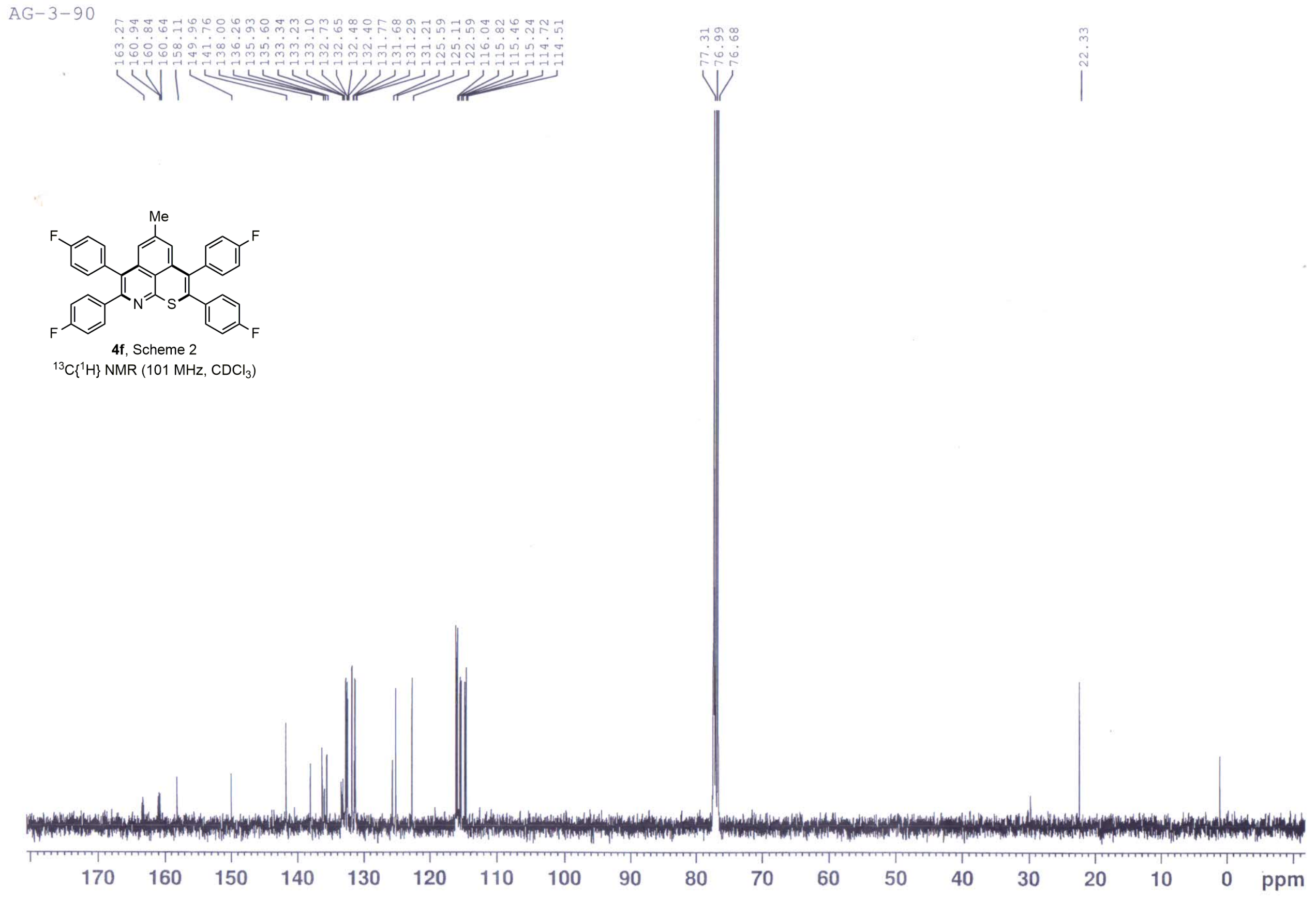


Ag-3-90

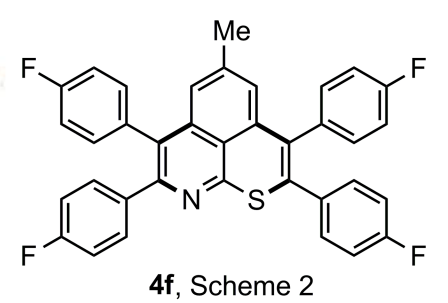

4f, Scheme 2

${ }^{19} \mathrm{~F} \mathrm{NMR} \mathrm{(376} \mathrm{MHz,} \mathrm{CDCl}_{3}$ )

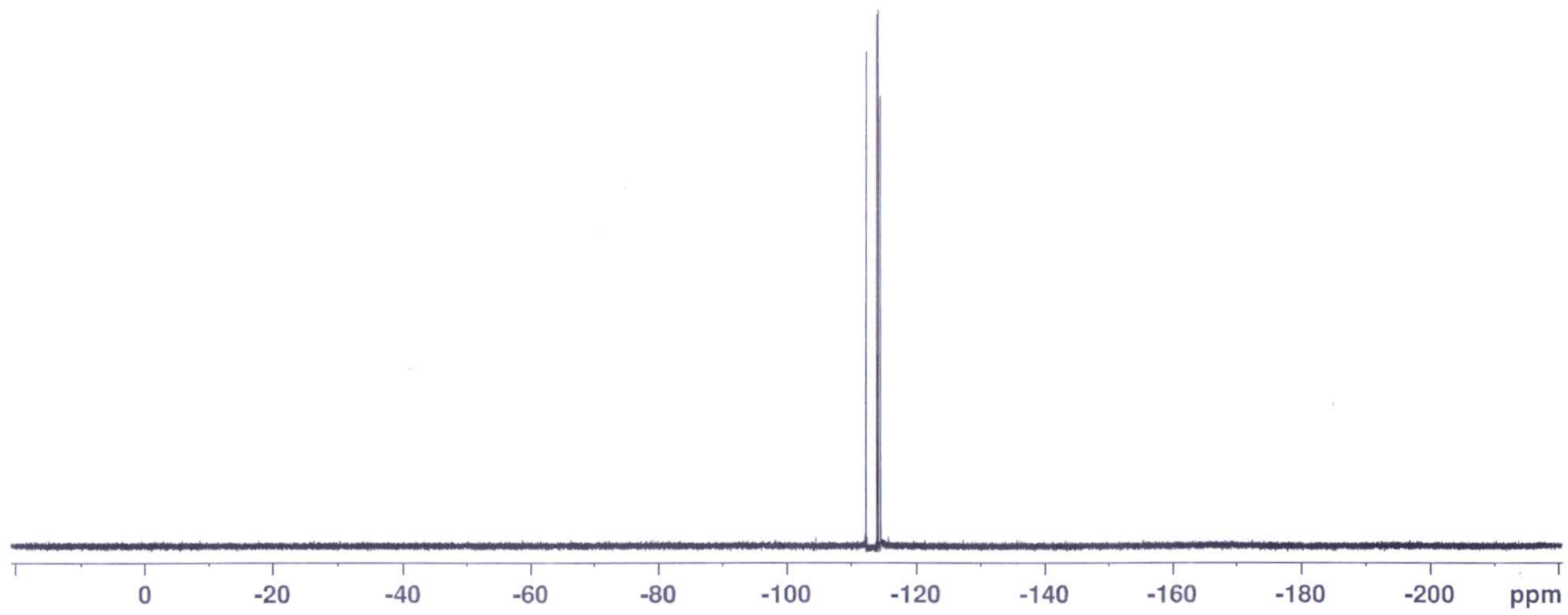




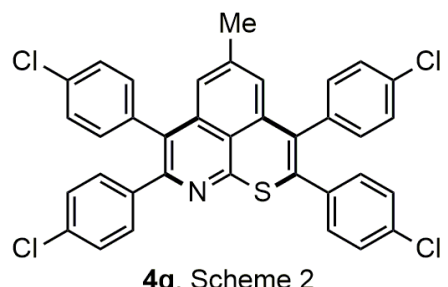

$4 \mathrm{~g}$, Scheme 2

${ }^{1} \mathrm{H} \mathrm{NMR}\left(400 \mathrm{MHz}, \mathrm{CDCl}_{3}\right)$

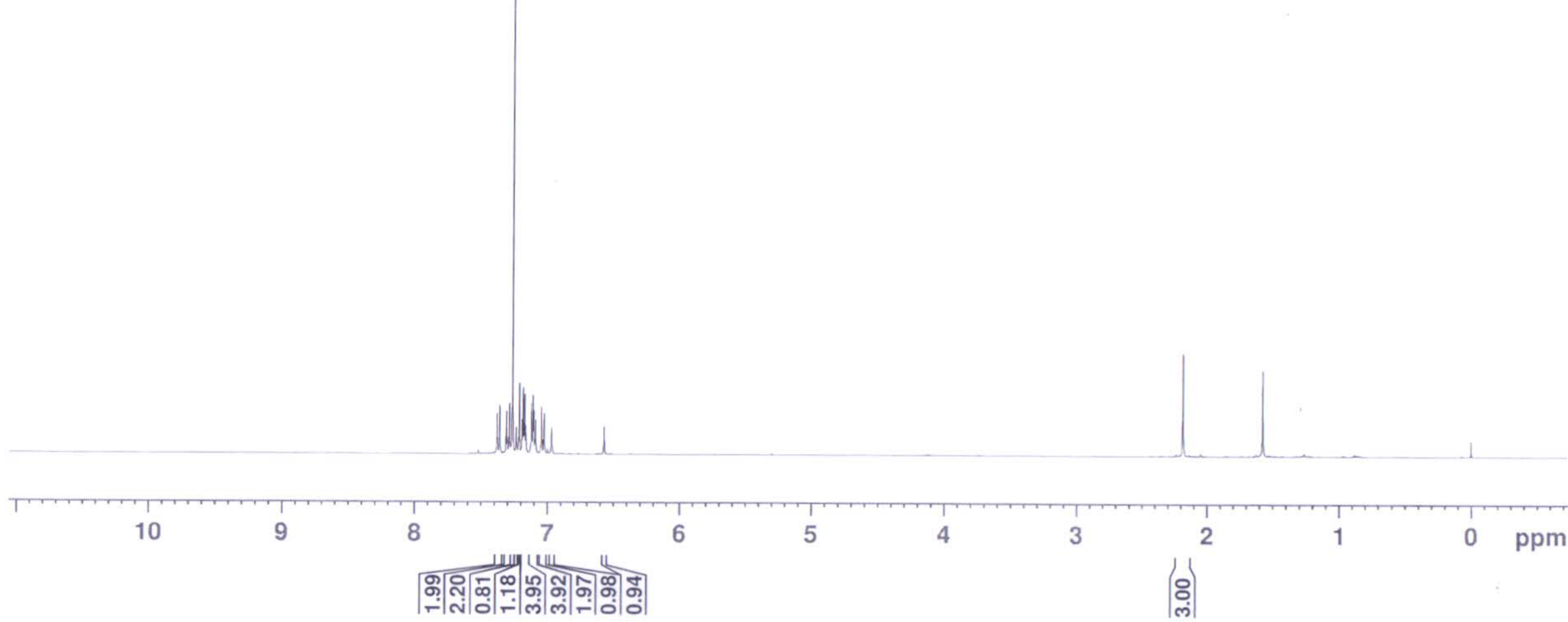




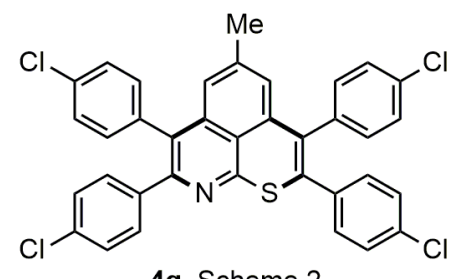

4g. Scheme 2

${ }^{13} \mathrm{C}\left\{{ }^{1} \mathrm{H}\right\}$ NMR $\left(101 \mathrm{MHz}, \mathrm{CDCl}_{3}\right)$
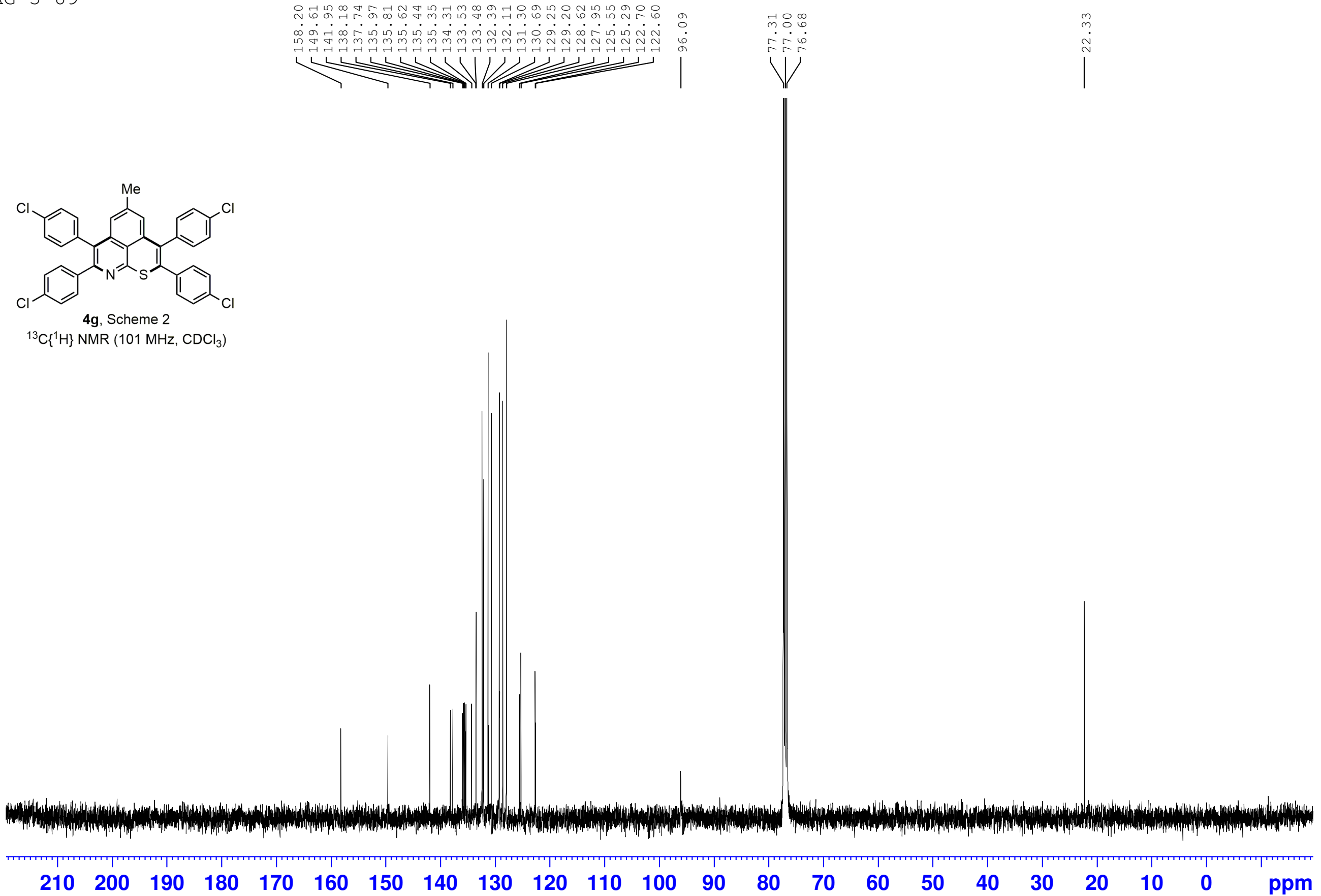
210200
$\begin{array}{llll}190 & 180 & 170 & 160\end{array}$
150
140130
$\begin{array}{lll}120 & 110 & 100\end{array}$
$90 \quad 80$
70

50

40




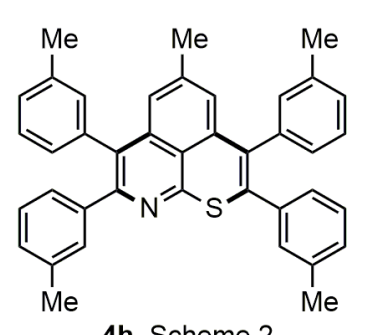

4h, Scheme 2

${ }^{1} \mathrm{H} \mathrm{NMR}\left(400 \mathrm{MHz}, \mathrm{CDCl}_{3}\right)$

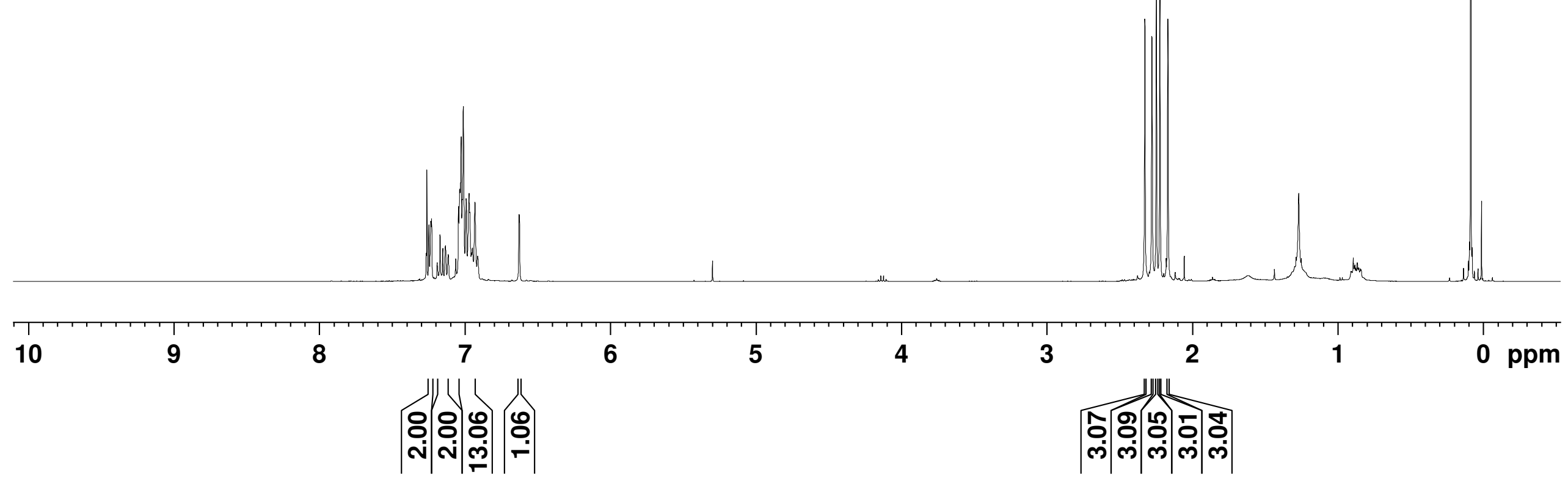




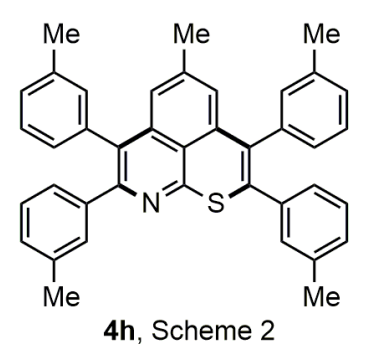

${ }^{13} \mathrm{C}\left\{{ }^{1} \mathrm{H}\right\} \mathrm{NMR}\left(101 \mathrm{MHz}, \mathrm{CDCl}_{3}\right)$

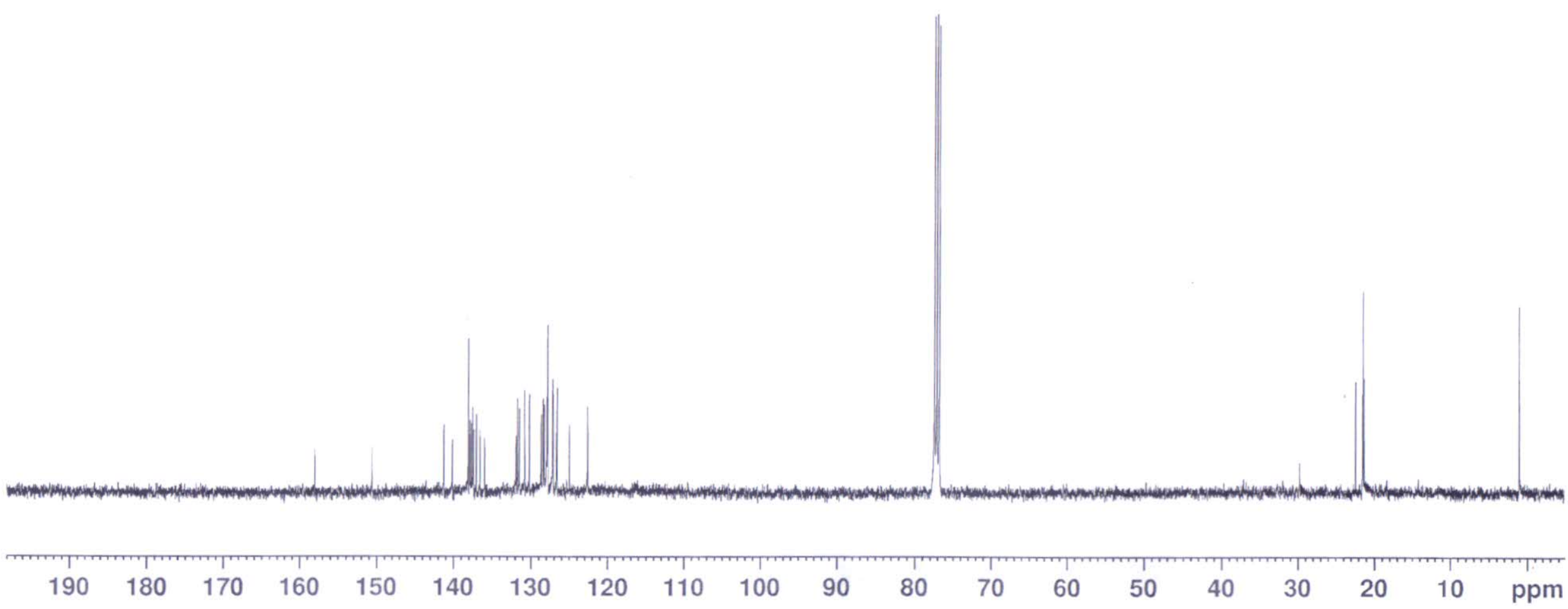




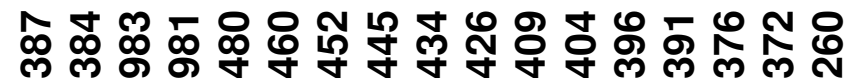 $\infty$ N N N N N N N N N N

MJ-155-15r

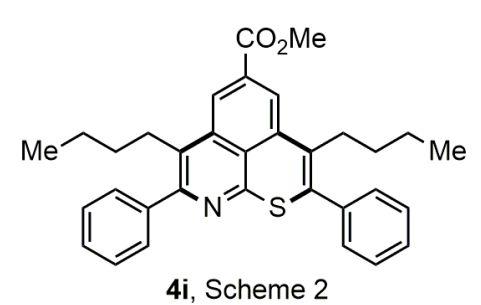

${ }^{1} \mathrm{H} \mathrm{NMR}\left(400 \mathrm{MHz}, \mathrm{CDCl}_{3}\right)$

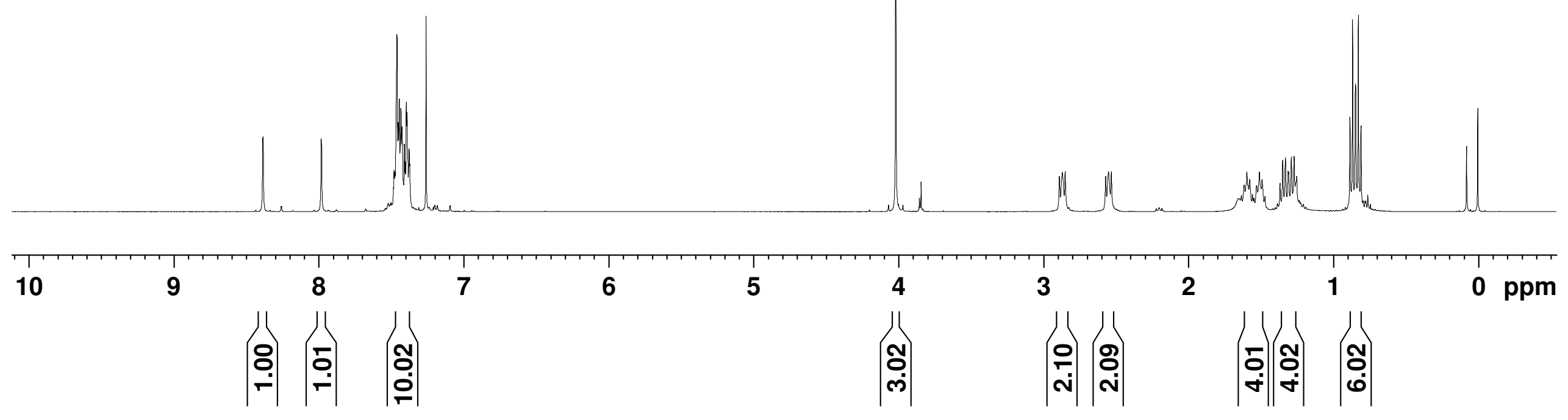




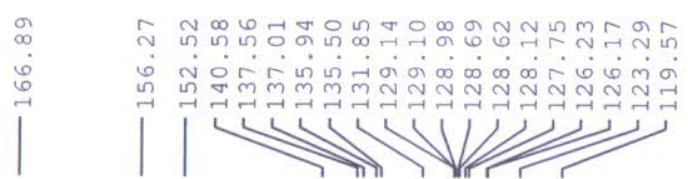

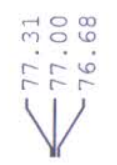

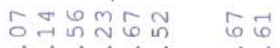

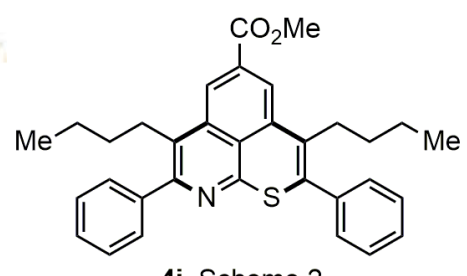

majji-155-15

4i, Scheme 2

${ }^{13} \mathrm{C}\left\{{ }^{1} \mathrm{H}\right\} \mathrm{NMR}\left(101 \mathrm{MHz}, \mathrm{CDCl}_{3}\right)$

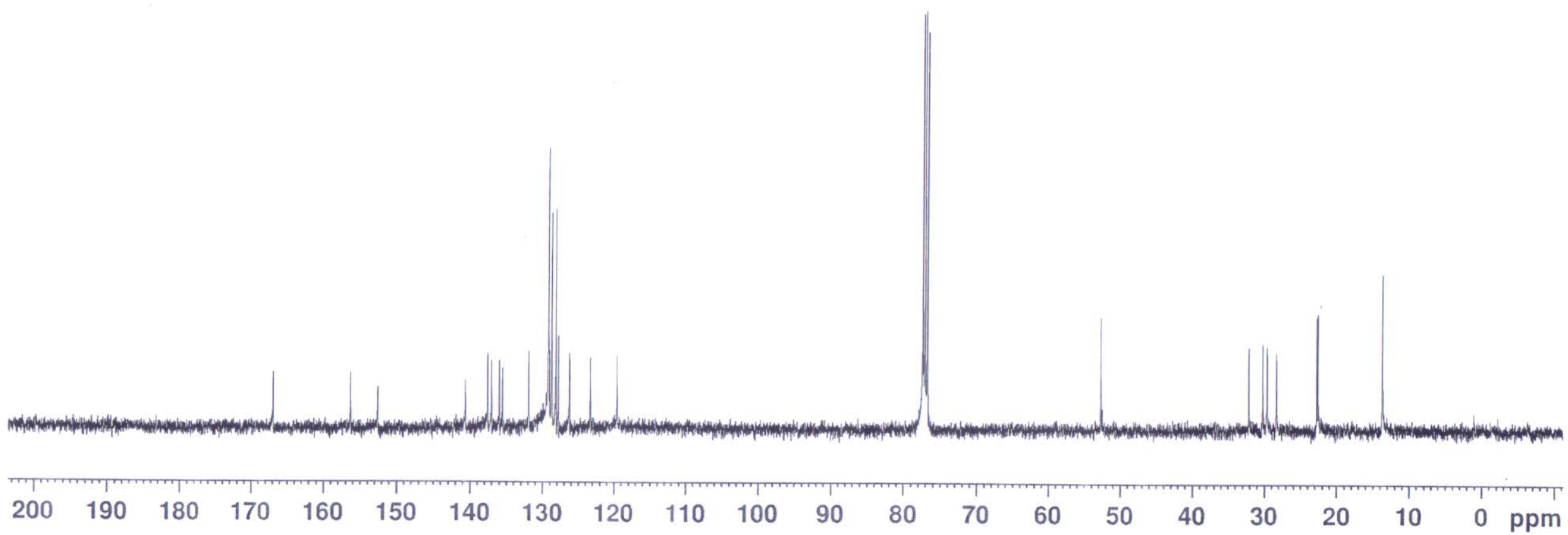




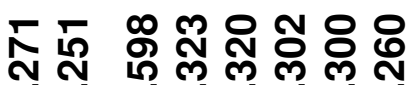

$\infty^{\infty} N N N N$

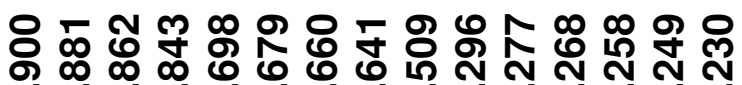

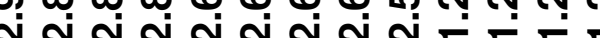

N N N N N

MJ-57-5-15-T2

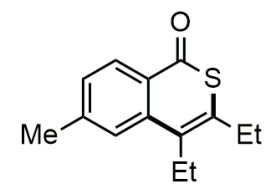

5a, Scheme

${ }^{1} \mathrm{H} \mathrm{NMR}\left(400 \mathrm{MHz}, \mathrm{CDCl}_{3}\right)$

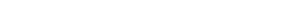




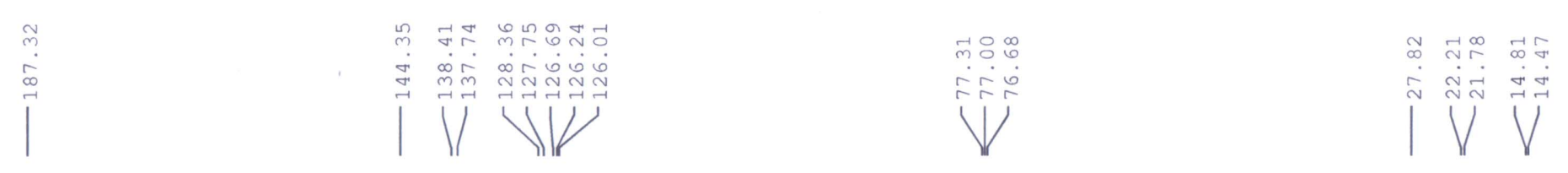

$m j-83-15$

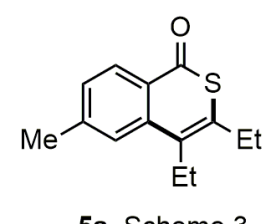

${ }^{13} \mathrm{C}\left\{{ }^{1} \mathrm{H}\right\}$ NMR (101 MHz, $\mathrm{CDCl}_{3}$ )

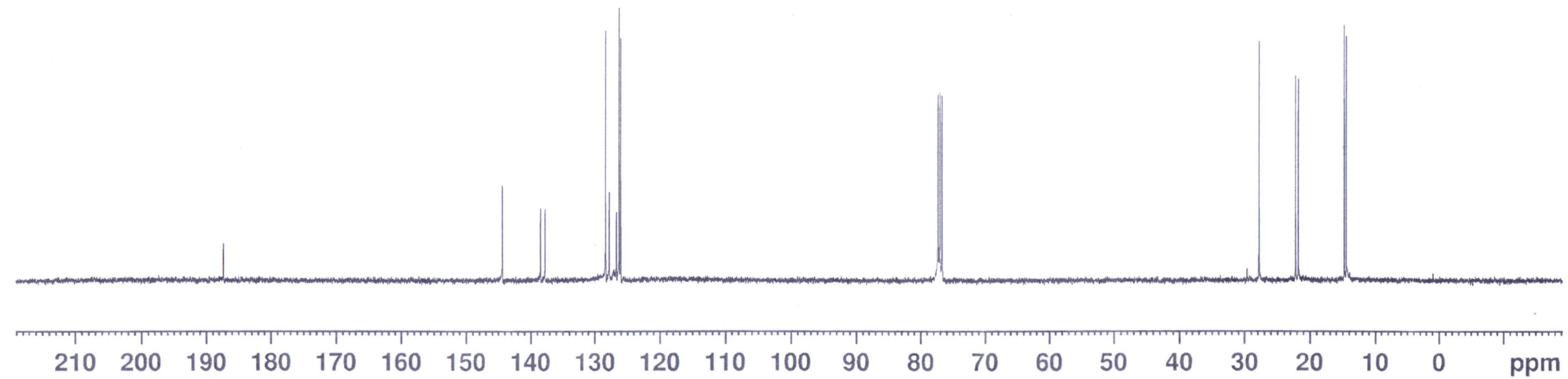




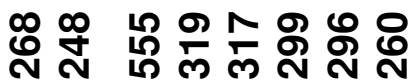

$\infty_{\infty}^{\infty} N N N$

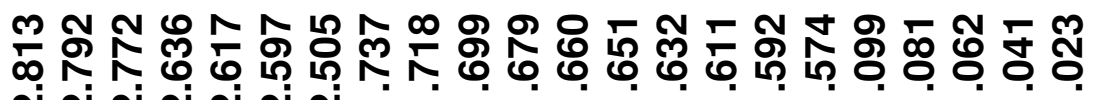

ง ส ง

MJ-84-15

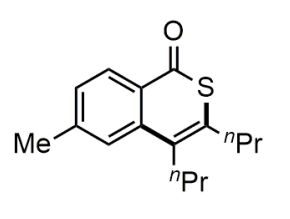

$\mathbf{5 b}$, Scheme 3

${ }^{1} \mathrm{H}$ NMR (400 MHz, $\mathrm{CDCl}_{3}$ )

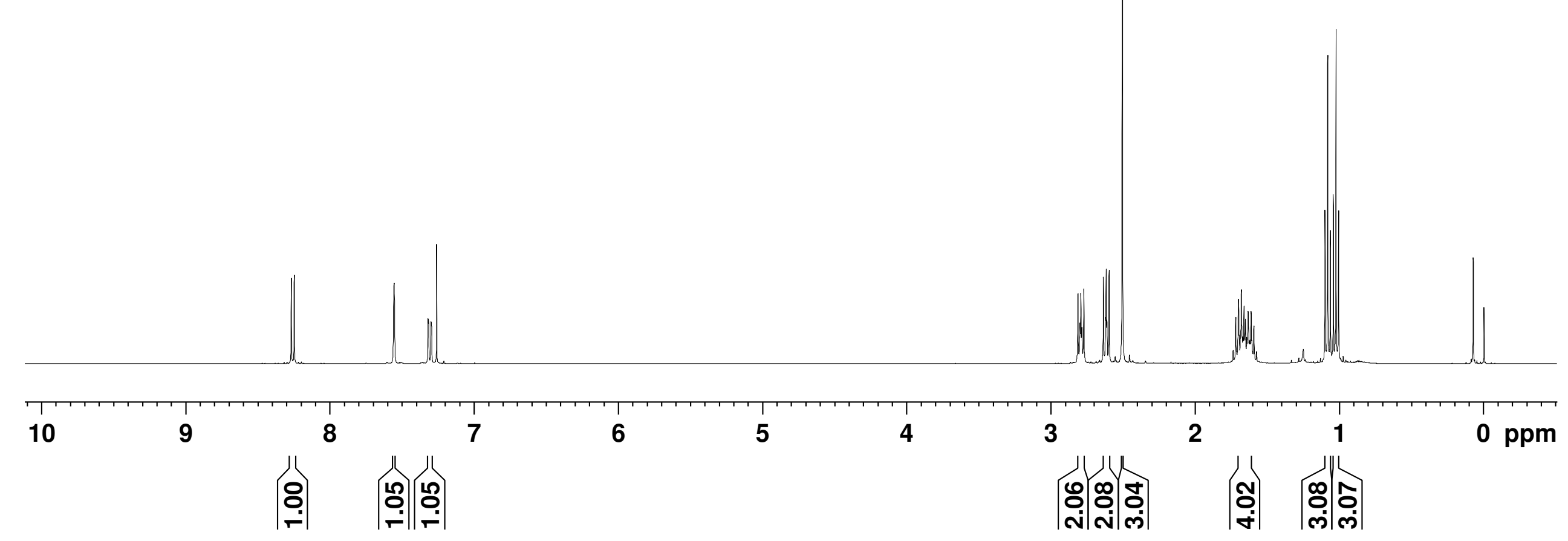




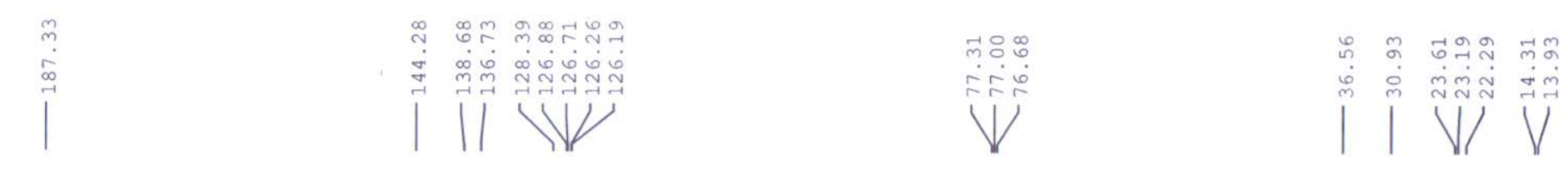

$m j-84-15$

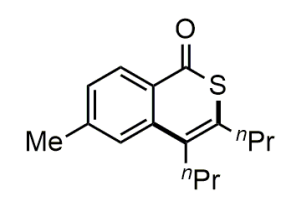

5b, Scheme 3

${ }^{13} \mathrm{C}\left\{{ }^{1} \mathrm{H}\right\}$ NMR $\left(101 \mathrm{MHz}, \mathrm{CDCl}_{3}\right)$
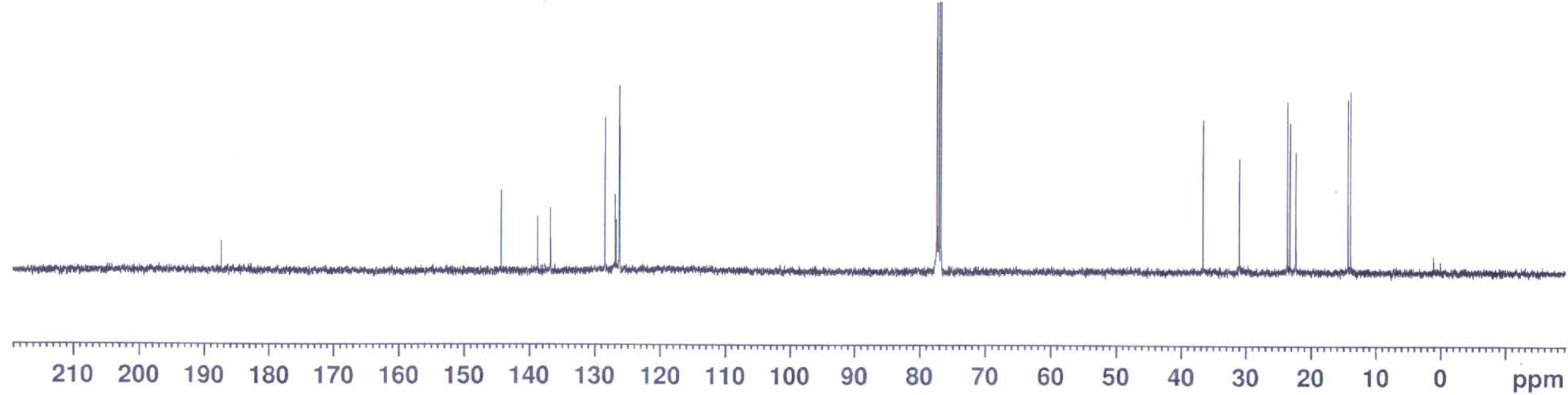

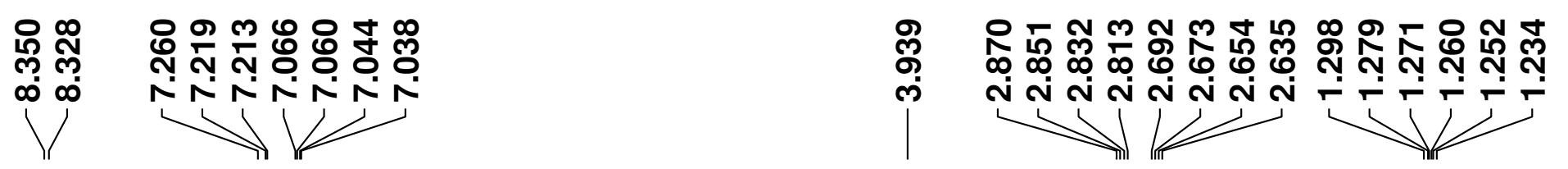

MJ-88-15
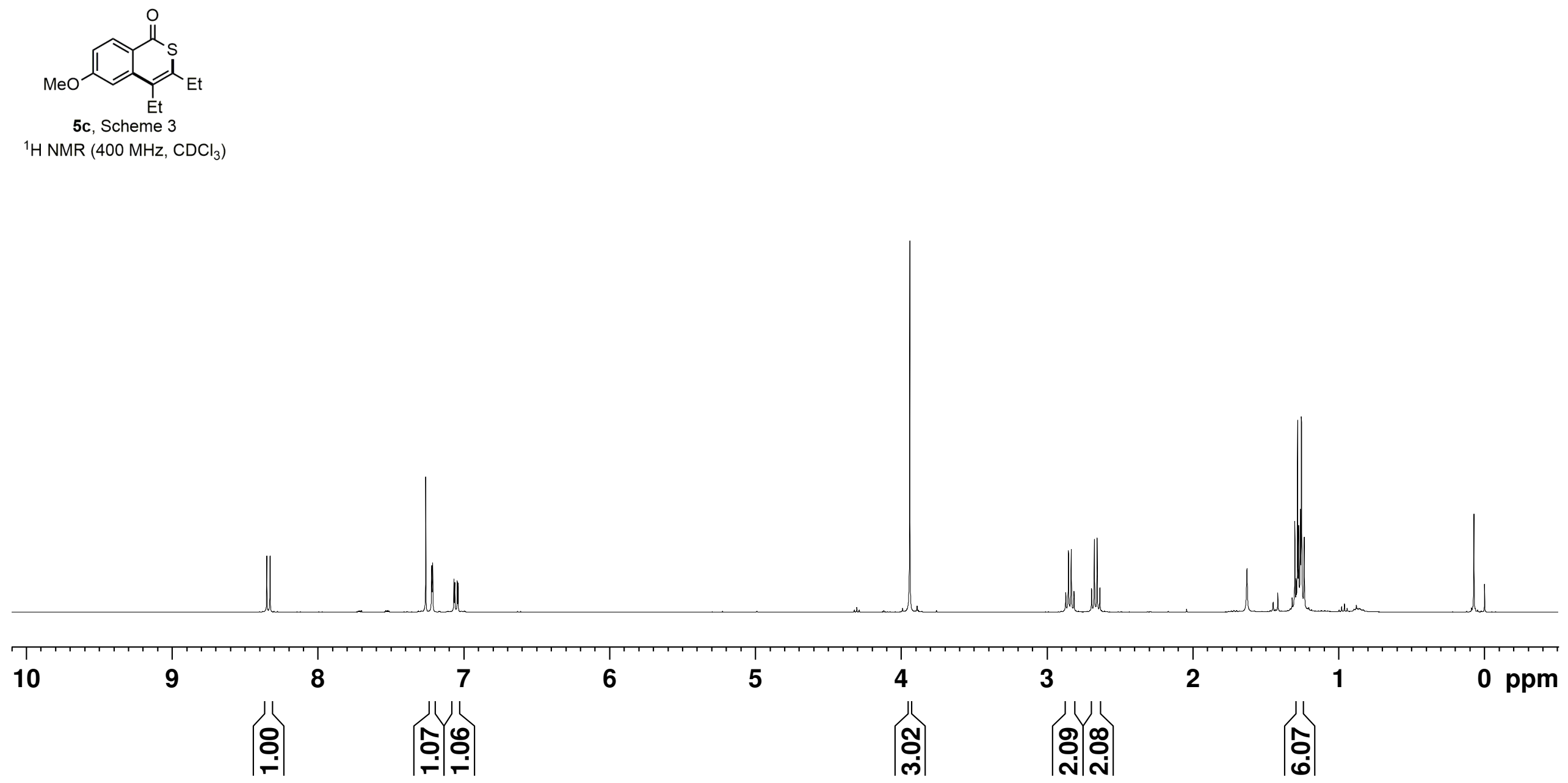


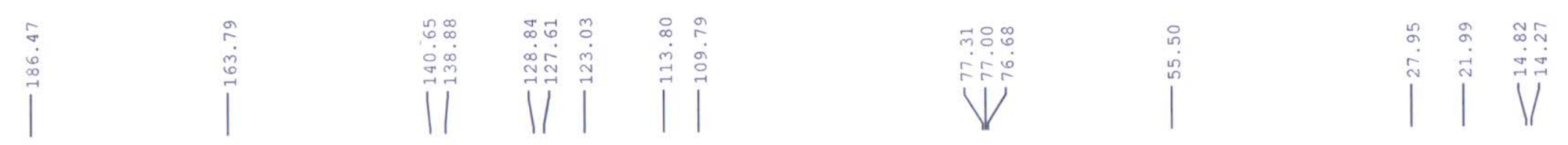

$m j-88-15$

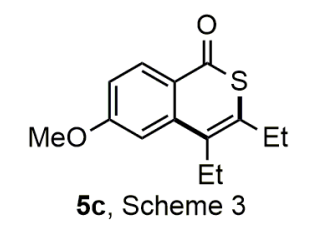

${ }^{13} \mathrm{C}\left\{{ }^{1} \mathrm{H}\right\}$ NMR (101 MHz, $\left.\mathrm{CDCl}_{3}\right)$

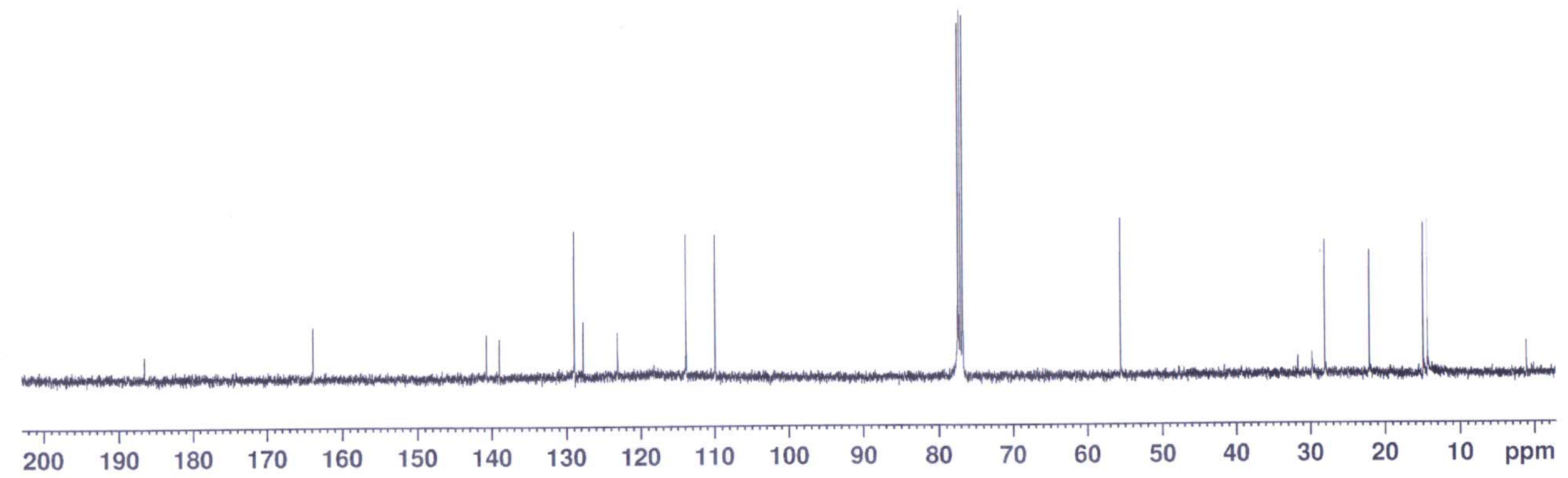




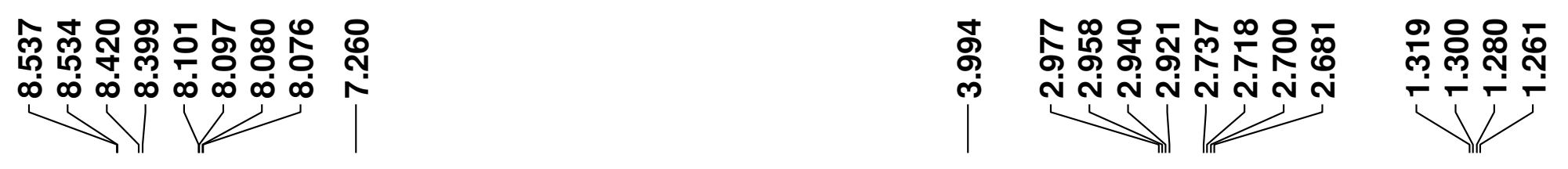

MJ-91-15
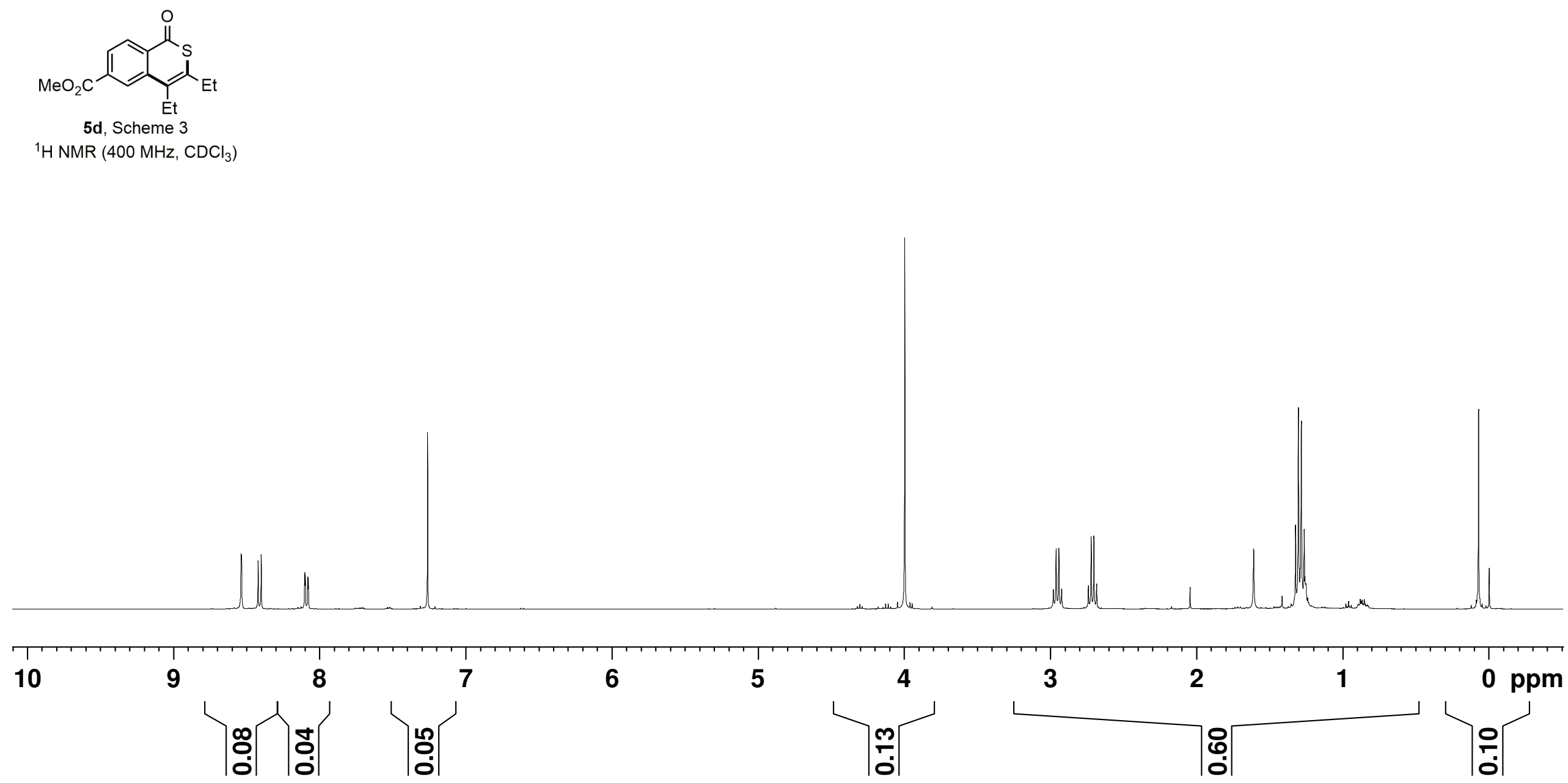


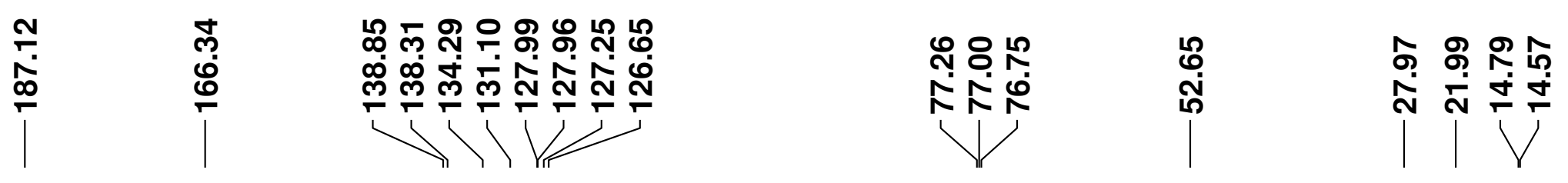

MJ-91-15

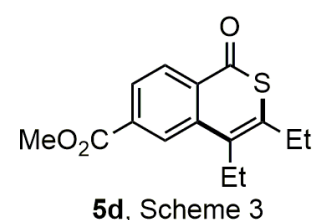

${ }^{13} \mathrm{C}\left\{{ }^{1} \mathrm{H}\right\} \mathrm{NMR}\left(101 \mathrm{MHz}, \mathrm{CDCl}_{3}\right)$

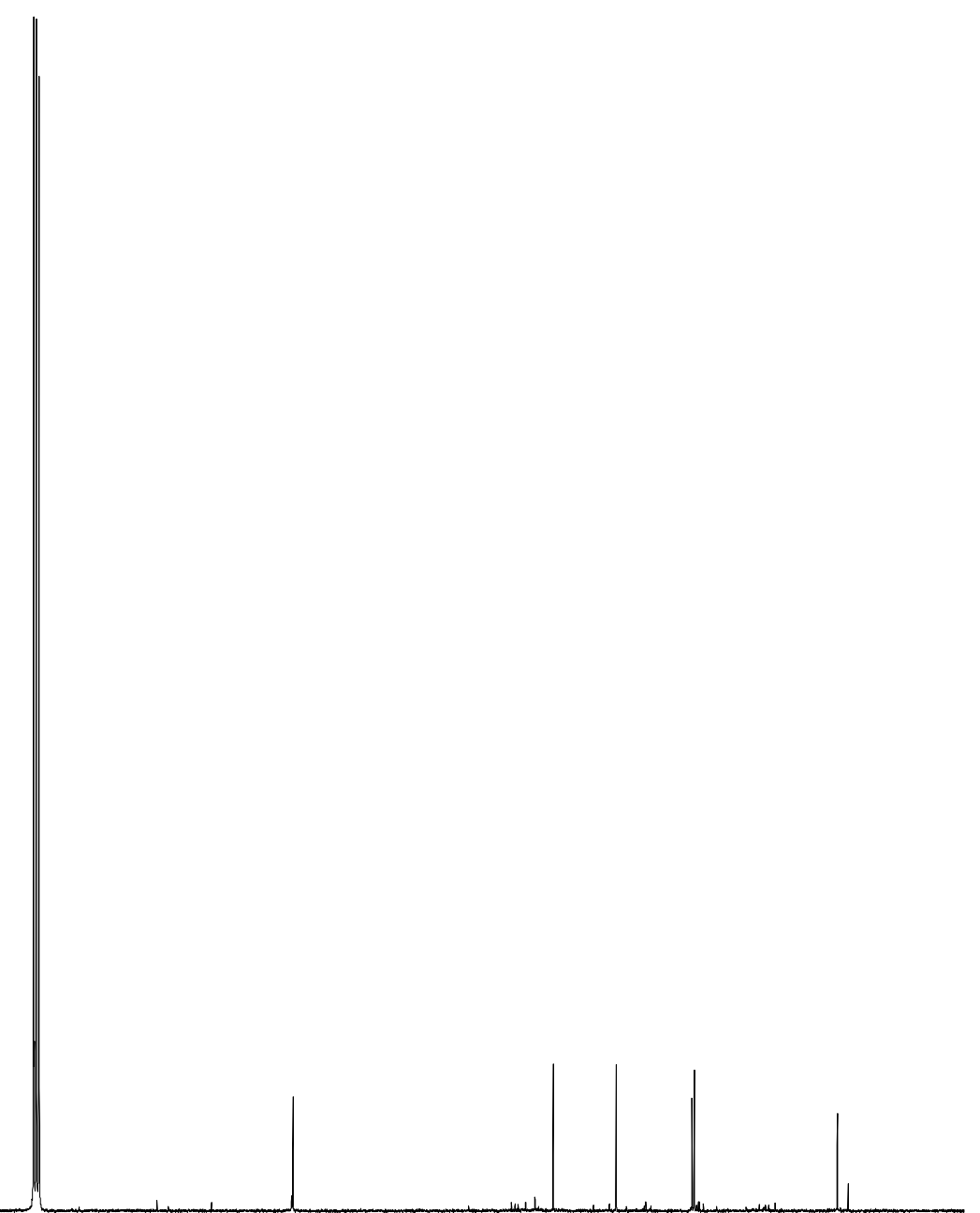

$\begin{array}{lllllllllllllllllllllll}210 & 200 & 190 & 180 & 170 & 160 & 150 & 140 & 130 & 120 & 110 & 100 & 90 & 80 & 70 & 60 & 50 & 40 & 30 & 20 & 10 & 0 & \mathrm{ppm}\end{array}$ 
MJ-104-15

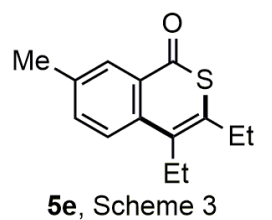

' $\mathrm{NMR}\left(400 \mathrm{MHz}, \mathrm{CDCl}_{3}\right)$

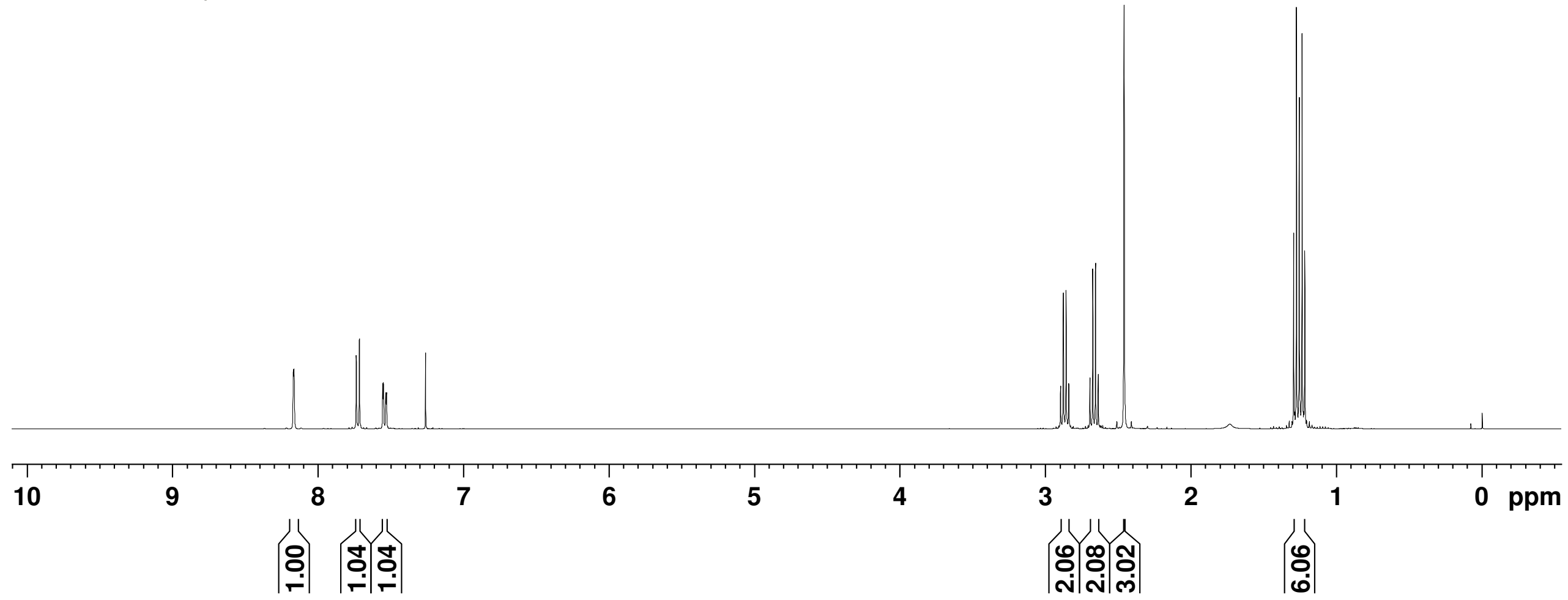



mj-104-15

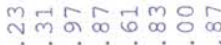

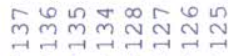
V/VV

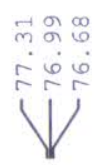

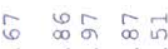
is

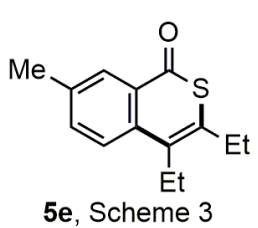

${ }^{13} \mathrm{C}\left\{{ }^{1} \mathrm{H}\right\}$ NMR $\left(101 \mathrm{MHz}, \mathrm{CDCl}_{3}\right)$

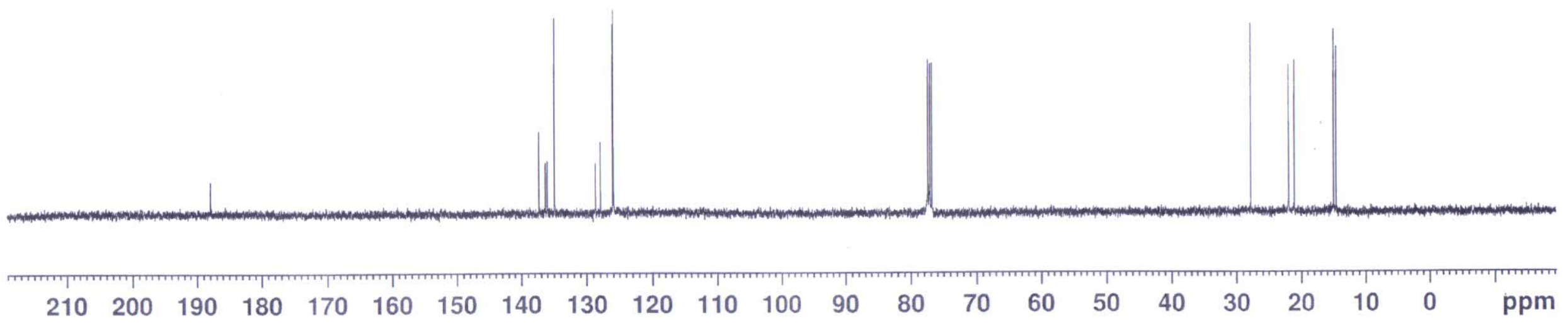




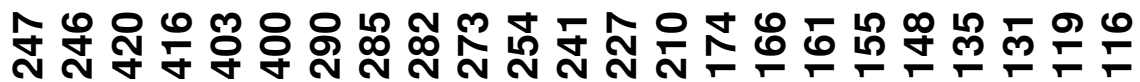

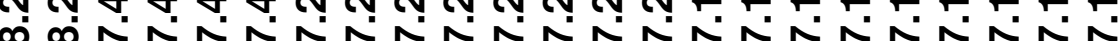

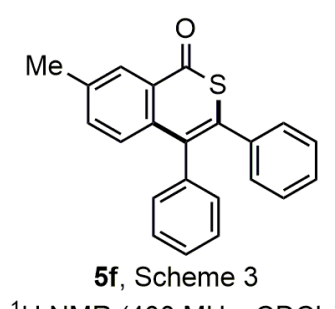

${ }^{1} \mathrm{H} \mathrm{NMR}\left(400 \mathrm{MHz}, \mathrm{CDCl}_{3}\right)$

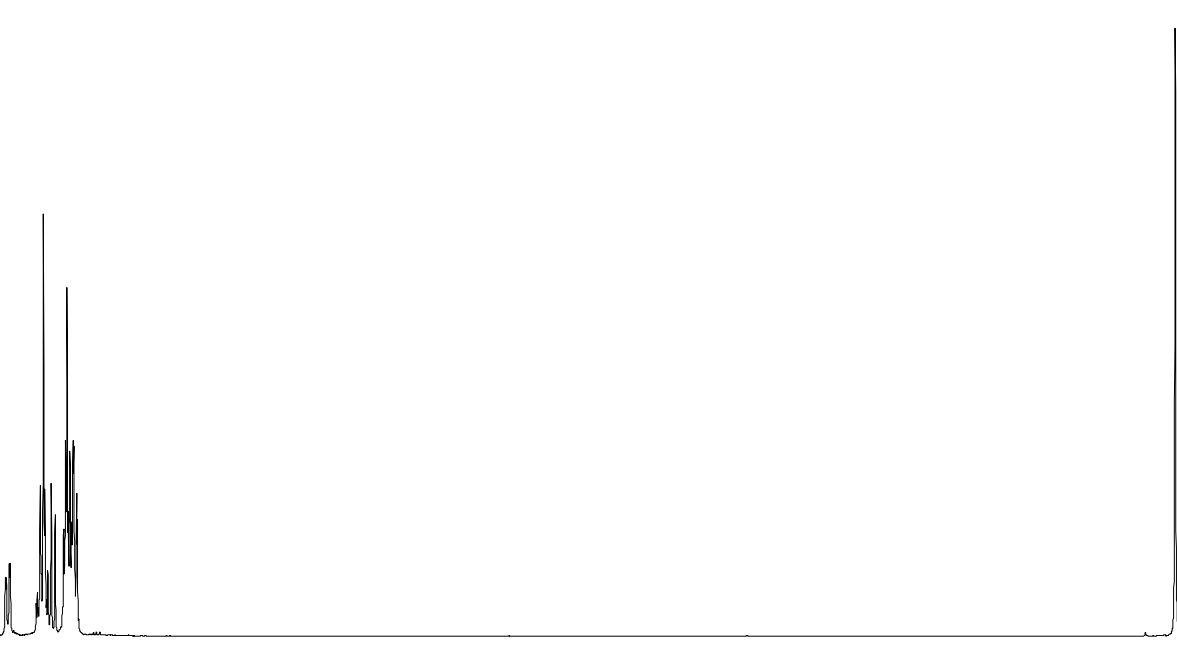

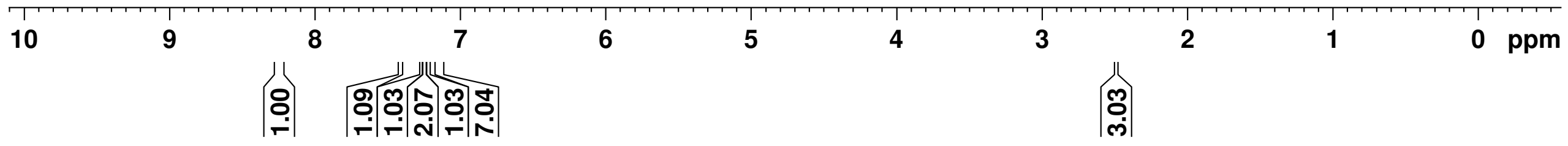




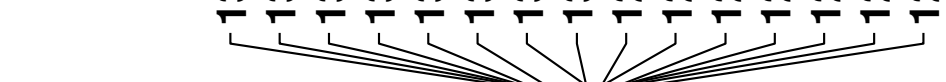

MJ-123-15

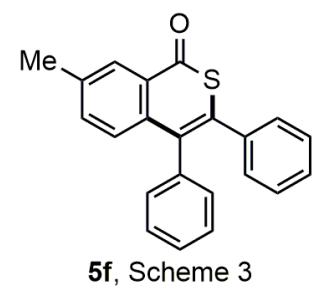

${ }^{13} \mathrm{C}\left\{{ }^{1} \mathrm{H}\right\}$ NMR $\left(101 \mathrm{MHz}, \mathrm{CDCl}_{3}\right)$

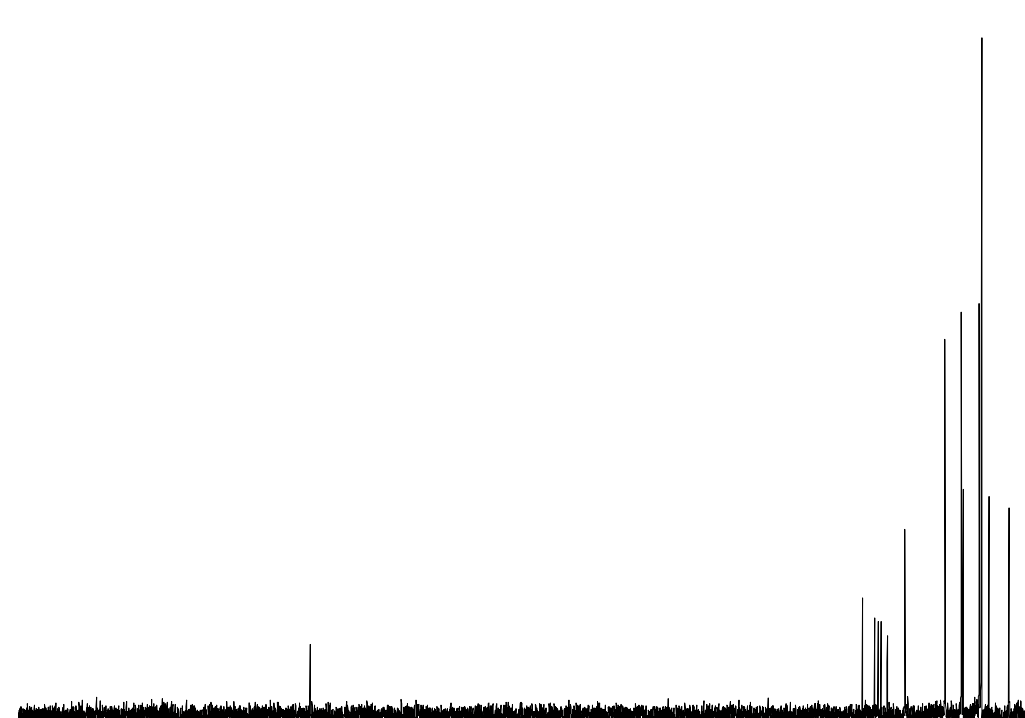

$\begin{array}{lllllllllllllllllllllll}210 & 200 & 190 & 180 & 170 & 160 & 150 & 140 & 130 & 120 & 110 & 100 & 90 & 80 & 70 & 60 & 50 & 40 & 30 & 20 & 10 & 0 & \text { ppm }\end{array}$ 


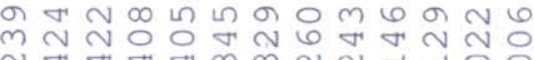

$0.46 r i x i r i x i n$

닌

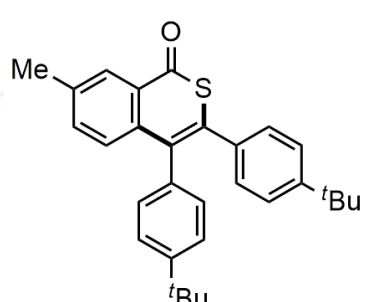

$\mathrm{H}$ NMR $\left(500 \mathrm{MHz}, \mathrm{CDCl}_{3}\right)$

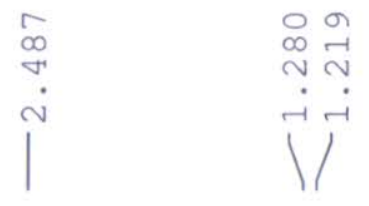

MJ-124-15

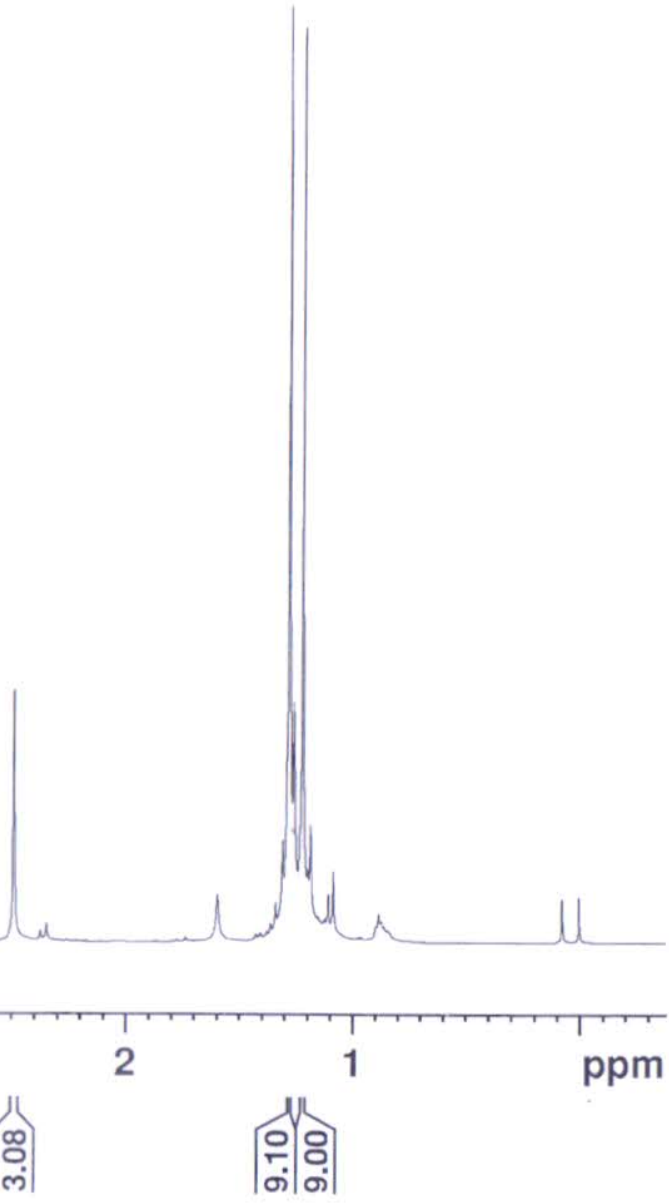




N

MJ-124-15

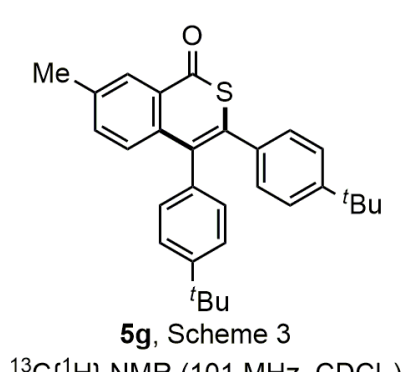

${ }^{13} \mathrm{C}\left\{{ }^{1} \mathrm{H}\right\}$ NMR $\left(101 \mathrm{MHz}, \mathrm{CDCl}_{3}\right)$

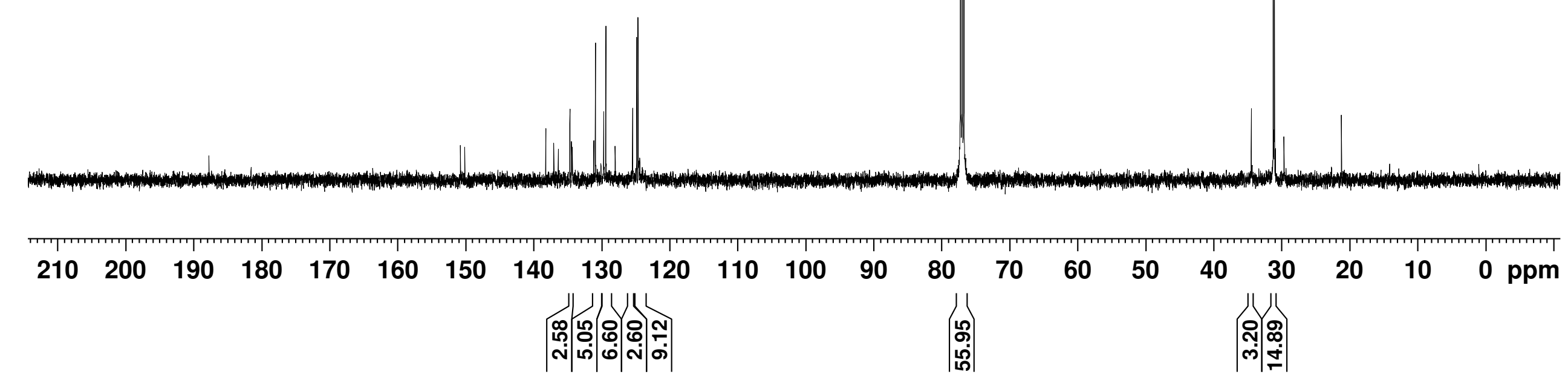




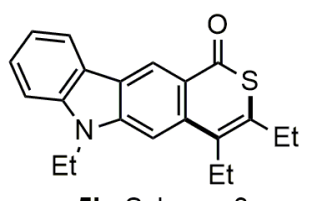

5h, Scheme 3

${ }^{1} \mathrm{H} \mathrm{NMR}\left(400 \mathrm{MHz}, \mathrm{CDCl}_{3}\right)$

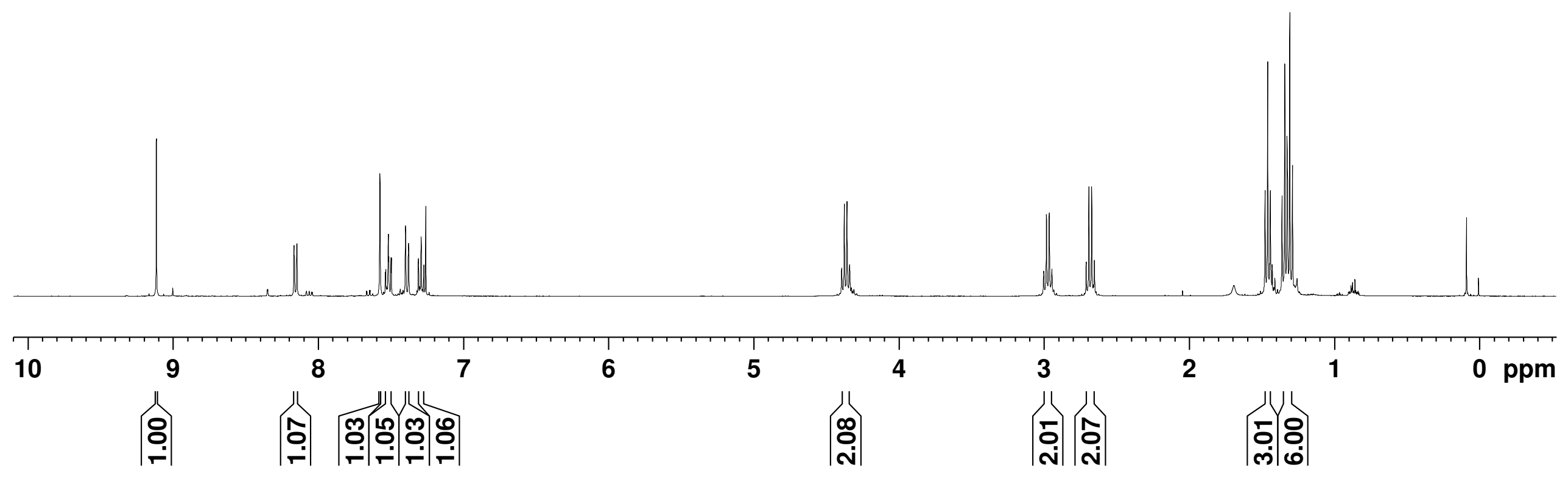



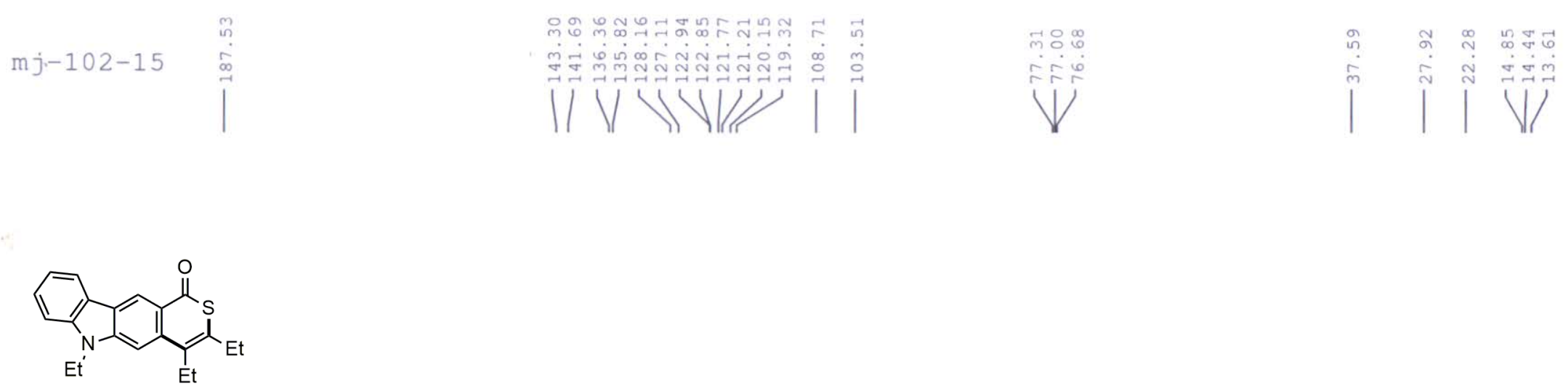

5h, Scheme 3

${ }^{13} \mathrm{C}\left\{{ }^{1} \mathrm{H}\right\} \mathrm{NMR}\left(101 \mathrm{MHz}, \mathrm{CDCl}_{3}\right)$

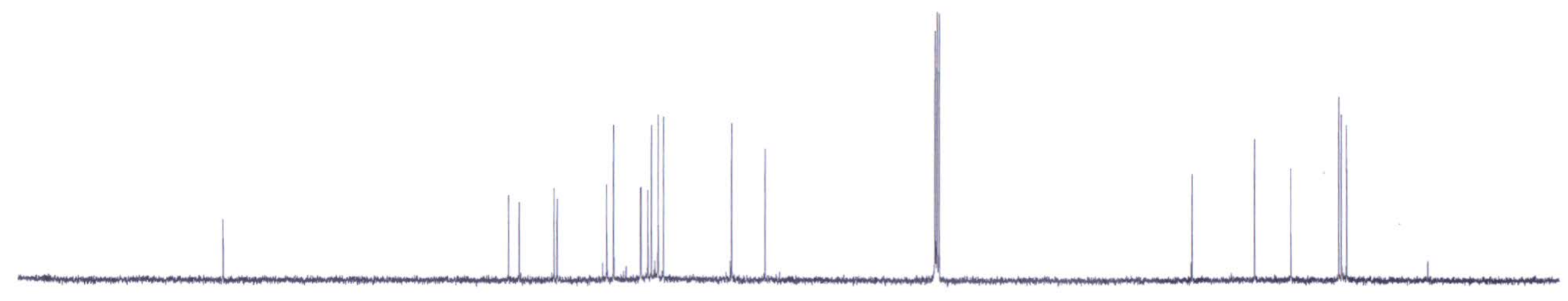

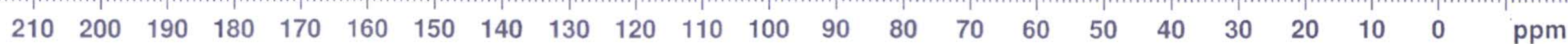




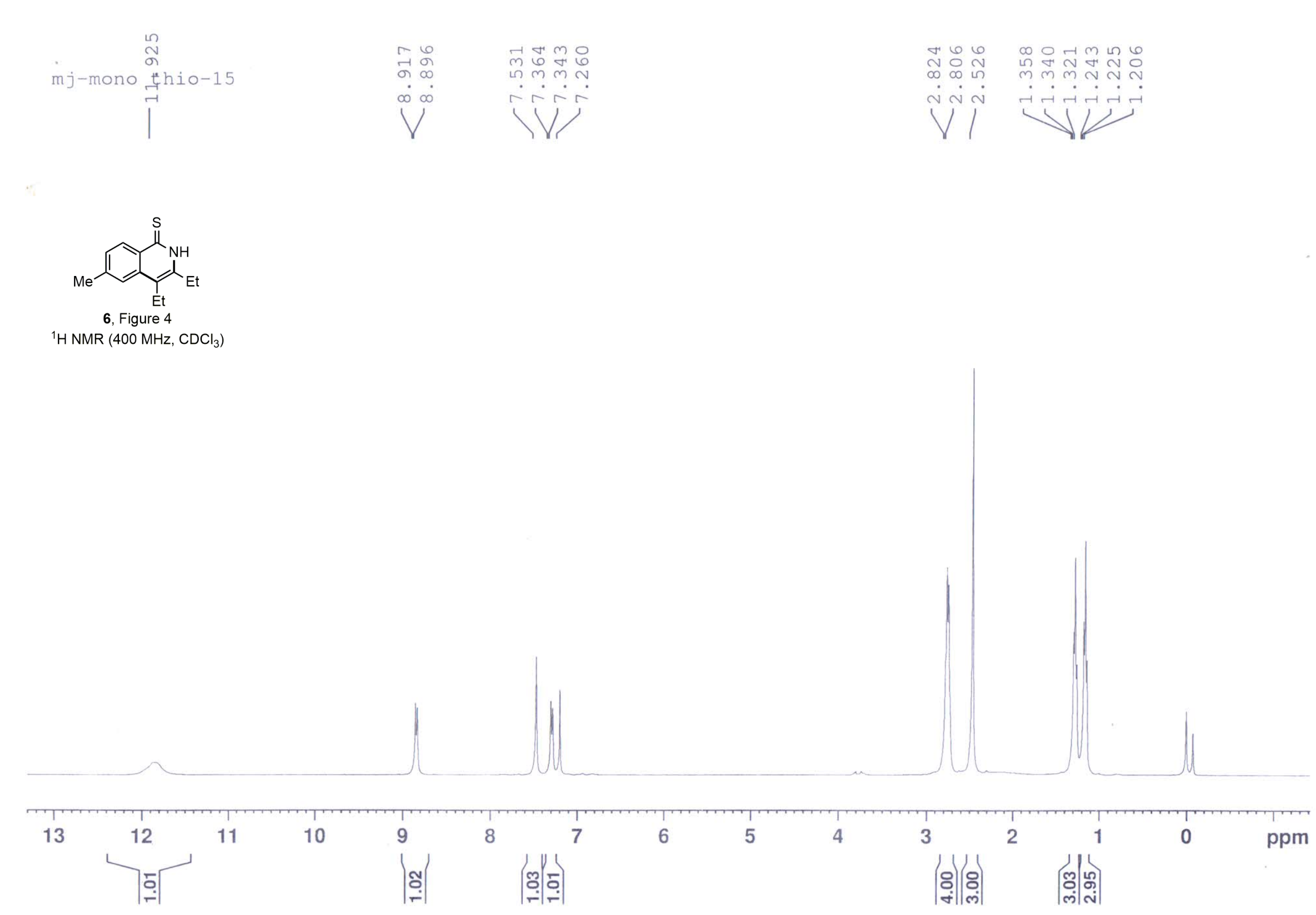


mj-mono-31-15

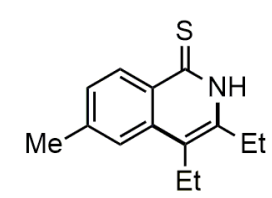

6. Figure 4

${ }^{13} \mathrm{C}\left\{{ }^{1} \mathrm{H}\right\}$ NMR (101 MHz, $\left.\mathrm{CDCl}_{3}\right)$

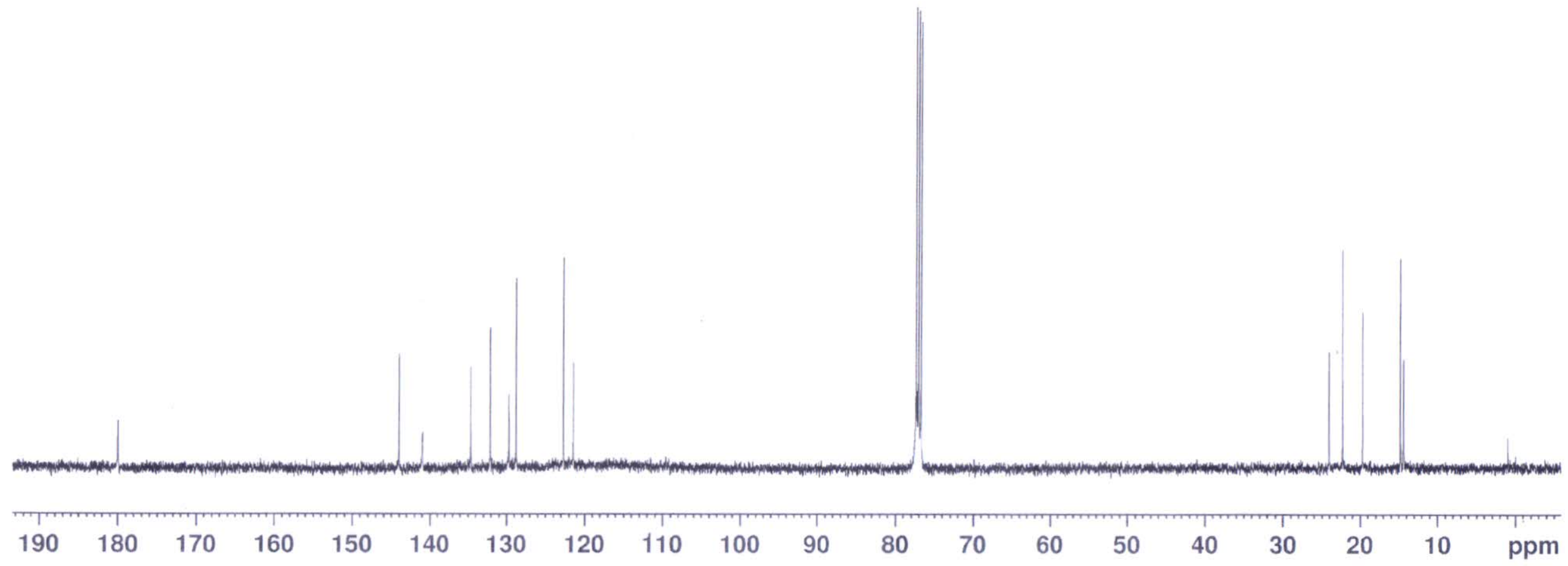




\section{UOH -SCHOOL OF CHEMISTRY -HRMS}

Analysis Info

Analysis Name

Method

Sample Name

D:IDatal2018lprof aksIDECImj-135-15a-r1.d

tune_low_Pos.m

mj-135-15a

Comment

Acquisition Parameter

Source Type ESI

Focus

Scan Begin

Not active

$50 \mathrm{~m} / \mathrm{z}$

$1500 \mathrm{~m} / \mathrm{z}$

Ion Polarity

Set Capillary

Set End Plate Offset

Set Collision Cell RF

Positive

$4200 \mathrm{~V}$

$-500 \mathrm{~V}$

Intens.

$\times 10^{5}$

8

$6-$

4

$2-$

231.5224

231
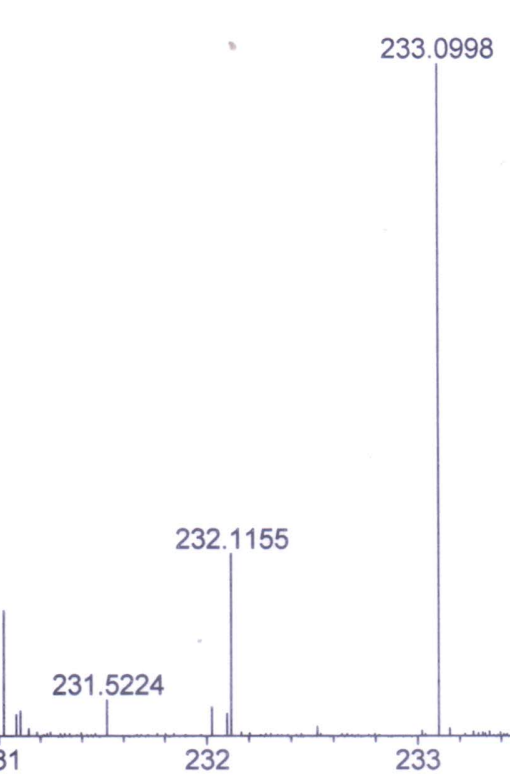

2.1155

235.1045

Acquisition Date

12/10/2018 12:11:09 PM

Operator

Instrument

UOH-Chemistry

maXis

10138

$\begin{array}{ll}\text { Set Nebulizer } & 0.3 \mathrm{Bar}\end{array}$

Set Dry Heater

Set Dry Gas

Set Divert Valve Waste

MS, 0.7-0.8min \#(40-46)

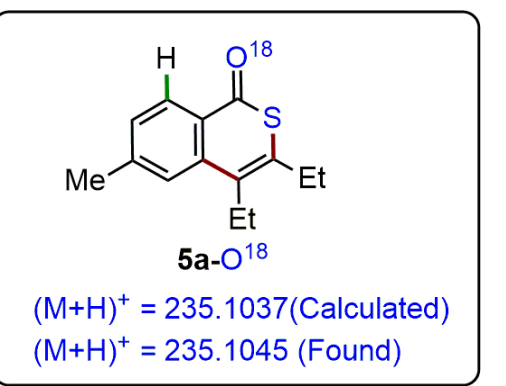

$(\mathrm{M}+\mathrm{H})^{+}=235.1045$ (Found)

Bruker Compass DataAnalysis 4.0

printed: $\quad$ 12/10/2018 3:27:13 PM

Page 1 of 1 


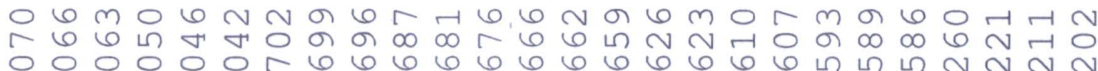

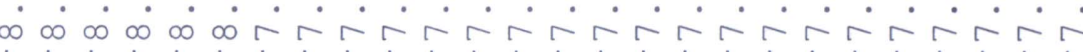

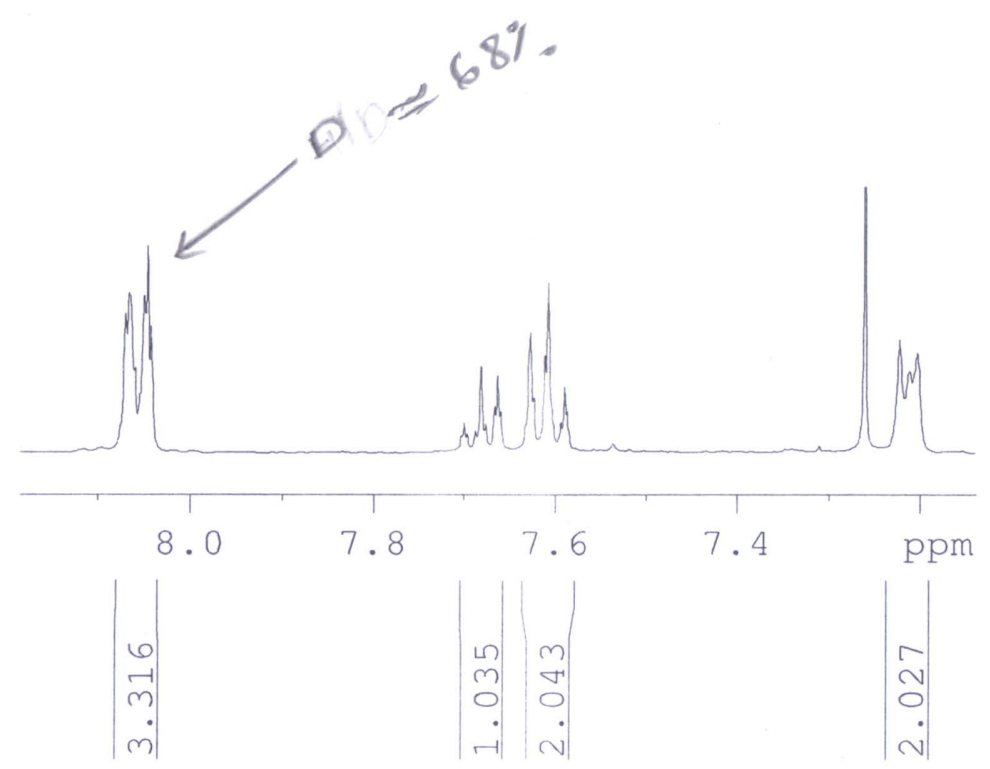

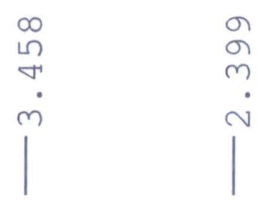

MJ-134-15
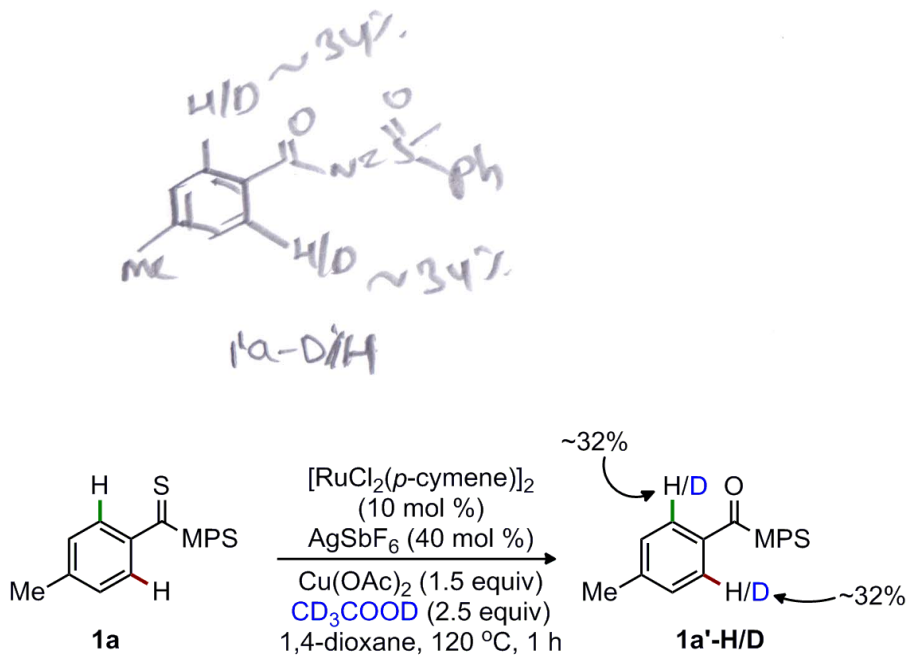

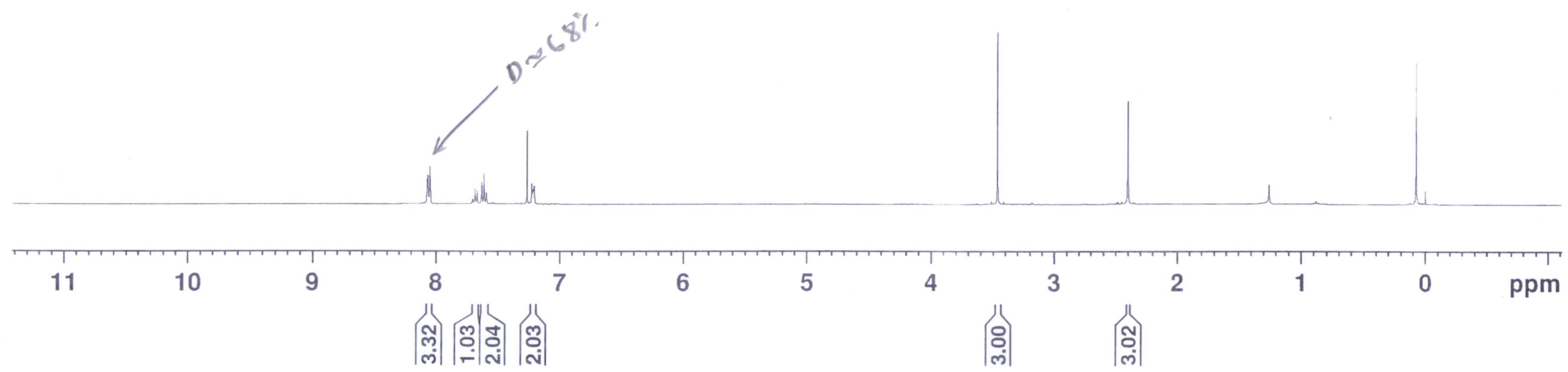




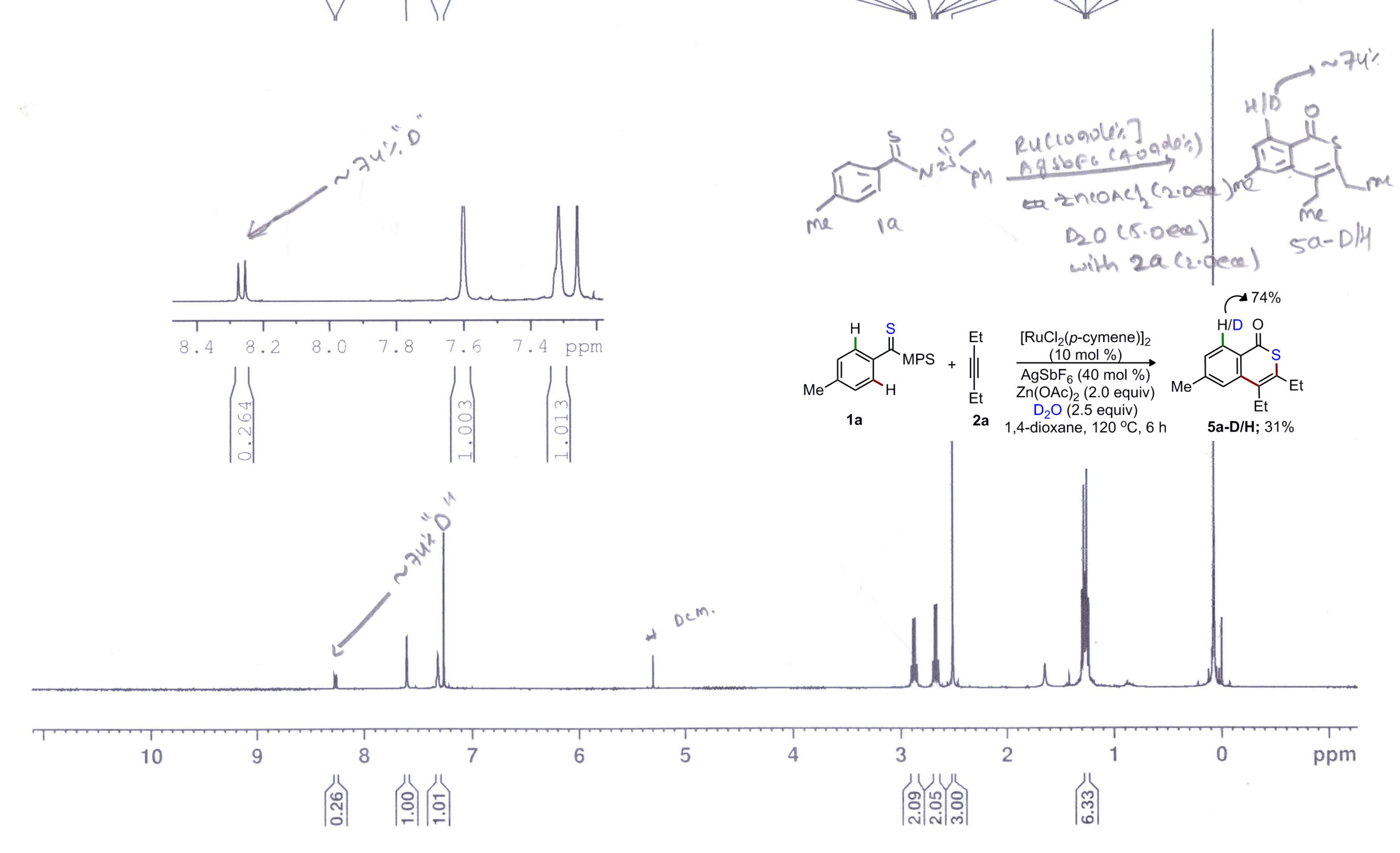




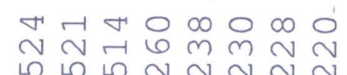

$\mathrm{MJ}-33-15$

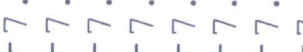

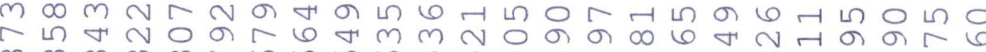

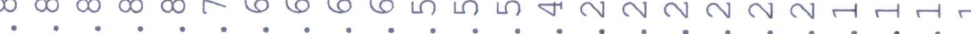
$\dot{v} \dot{\sim} \dot{\sim} \dot{\sim} \dot{\sim} \dot{\sim} \dot{\sim} \dot{\sim} \dot{\sim} \dot{\sim}$
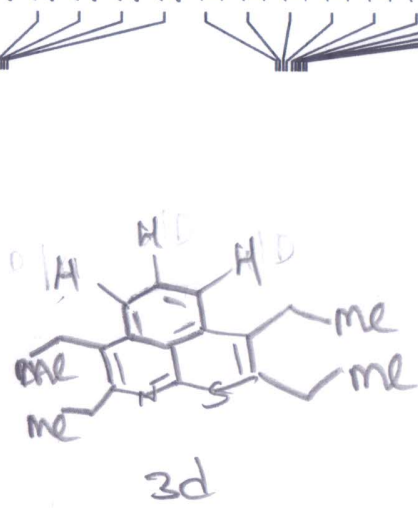

$\underbrace{S P P S}_{1 d}+$ $\underset{\left[\operatorname{RuCl}_{2}(p \text {-cymene })\right]_{2}}{(10 \mathrm{~mol} \%)}$
$\underset{\mathrm{AgSbF}}{6}(40 \mathrm{~mol} \%)$
$\mathrm{Cu}(\mathrm{OAc})_{2}(1.5$ equiv $)$

$2 a$ 1,4-dioxane, $120^{\circ} \mathrm{C}, 30 \mathrm{~min}$

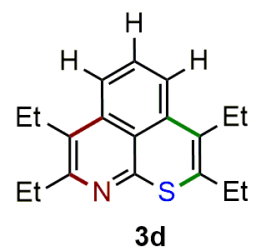

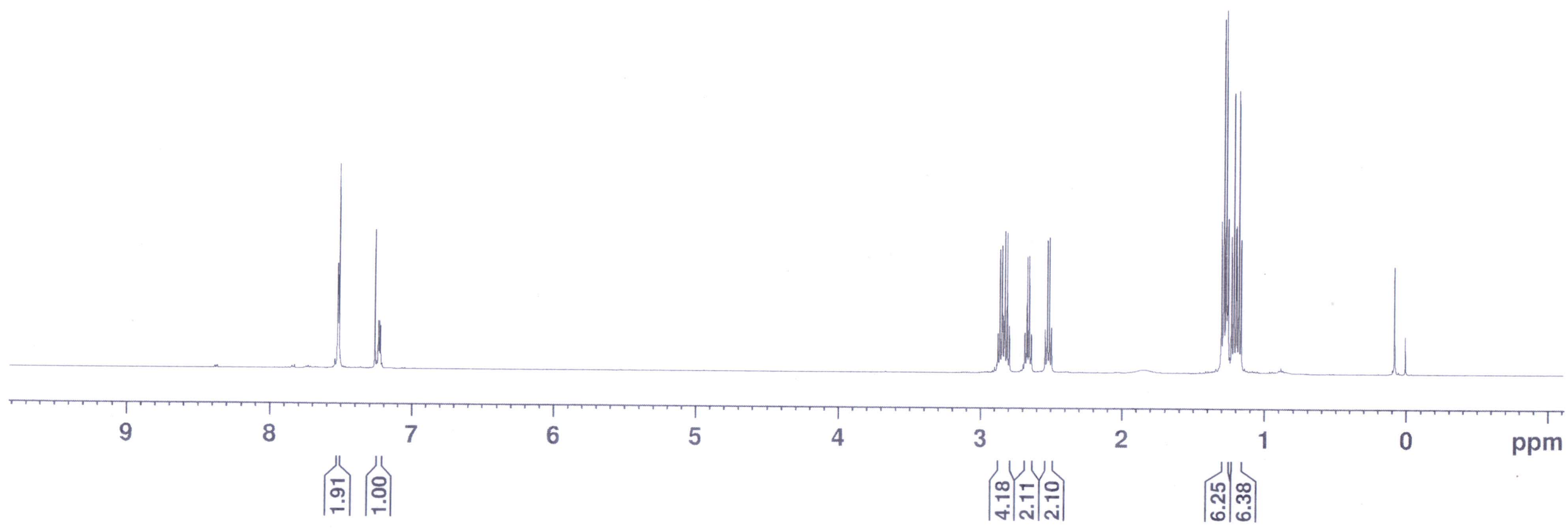




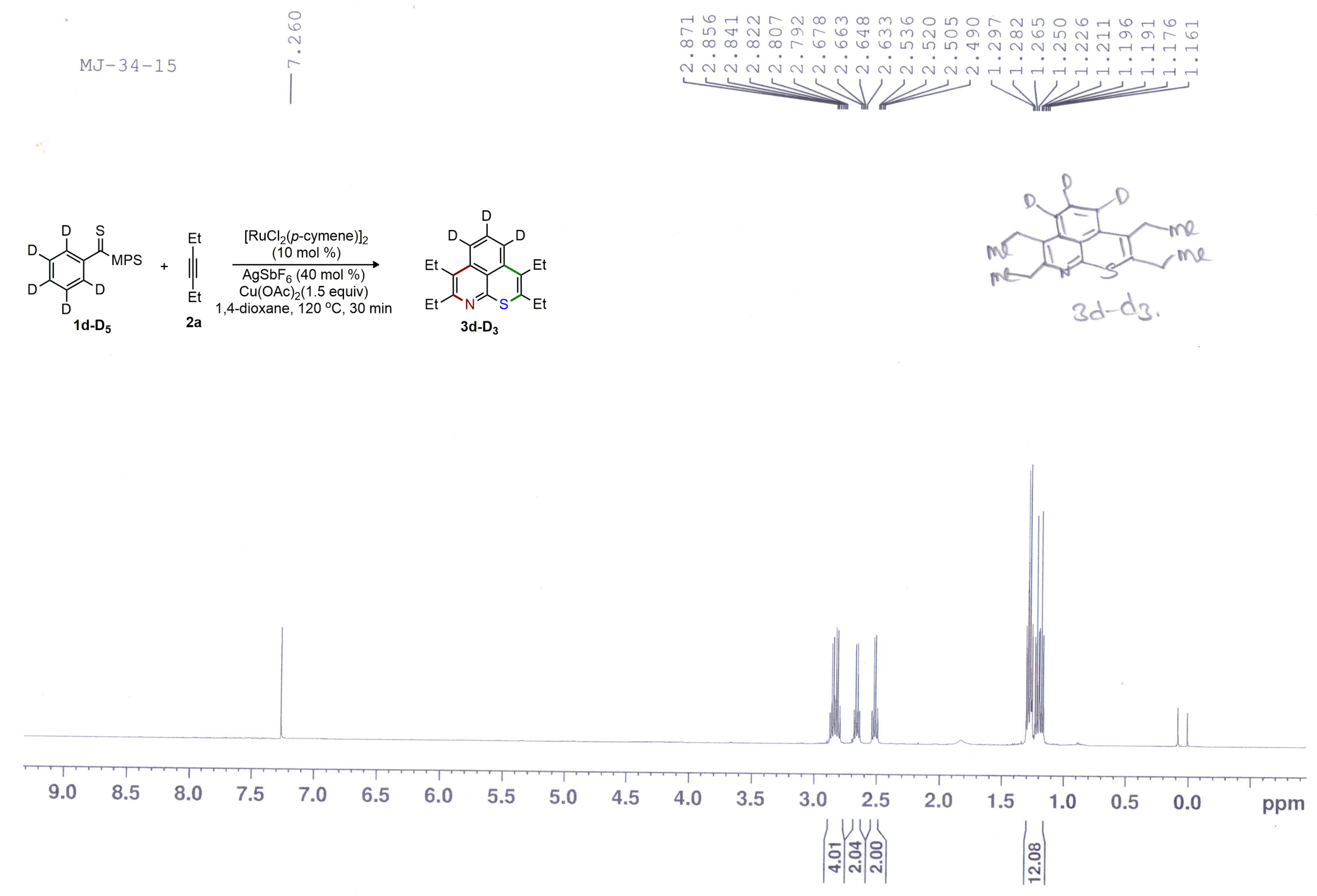




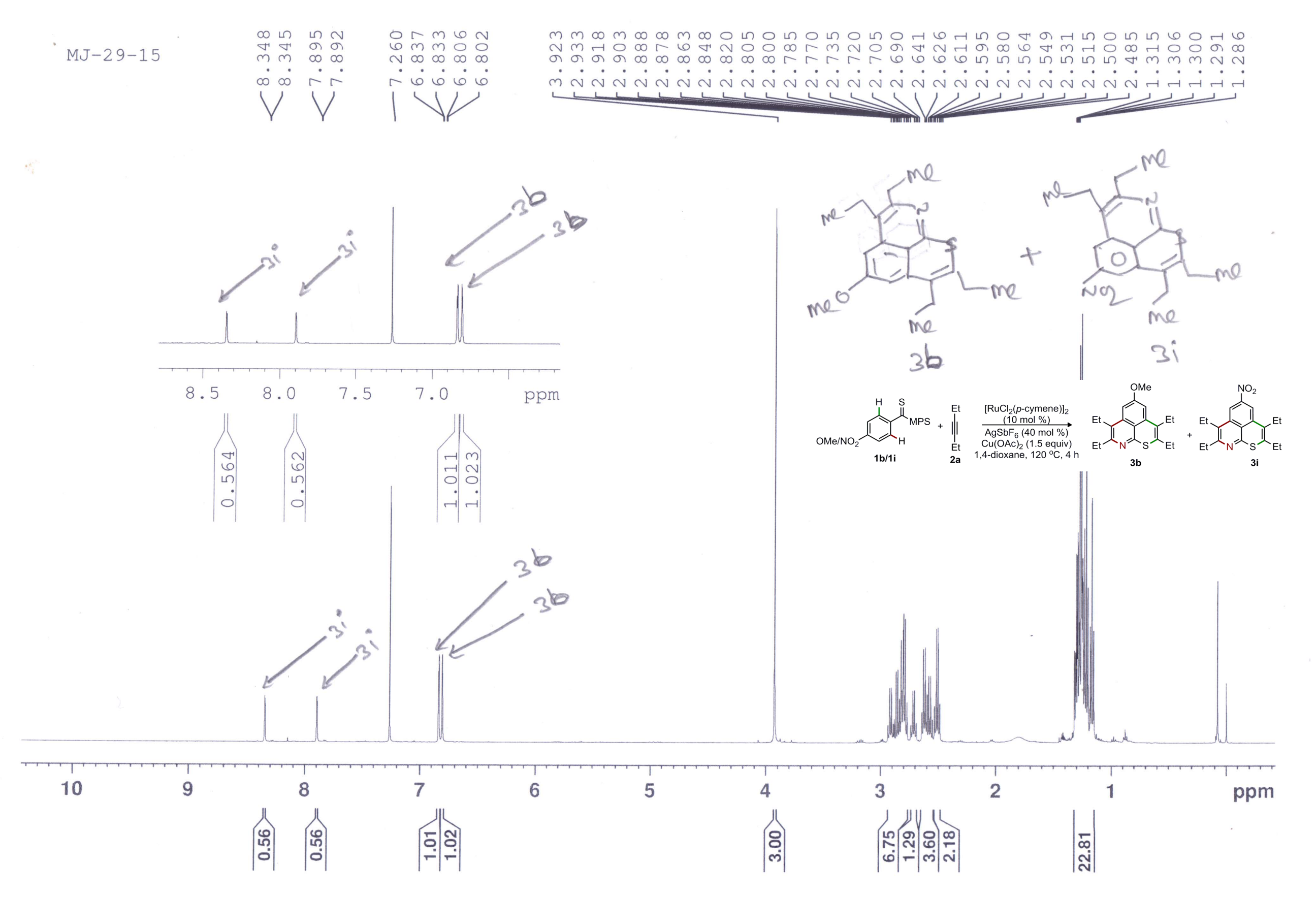




\section{UOH -SCHOOL OF CHEMISTRY -HRMS}

\section{Analysis Info}

Analysis Name

Method

Sample Name

D:IDatal2018lprof aksIDECI137-15.d

TL-P.m

$137-15$

Comment

Acquisition Parameter

Source Type ESI

Focus

Scan Begin

Not active

$50 \mathrm{~m} / \mathrm{z}$

(n) $600 \mathrm{~m} / \mathrm{z}$

$\times 10^{5}$

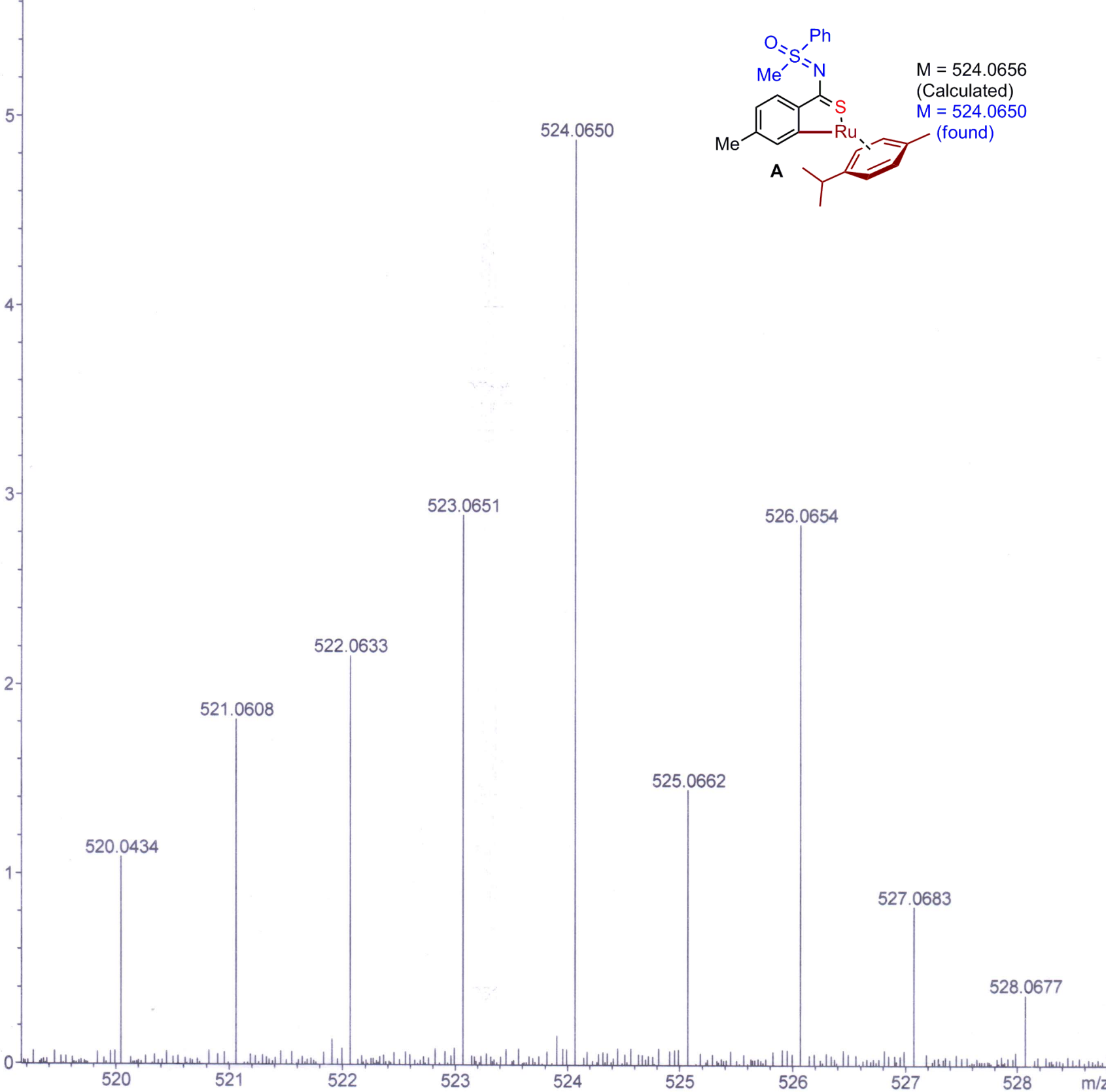

Bruker Compass DataAnalysis 4.0
Acquisition Date $\quad$ 12/11/2018 5:29:03 PM

Operator UOH-Chemistry

Instrument maXis

10138 


\section{UOH -SCHOOL OF CHEMISTRY -HRMS}

Analysis Info

Analysis Name

Method

Sample Name

\section{Comment}

2018lprof aksIDECImj-135-15a-r1.d

une low_Pos.m

$\mathrm{mj}-1 \overline{3} 5-1 \overline{5} \mathrm{a}$

Acquisition Date

Operator

Acquisition Parameter

Source Type ES

Focus

Not active

$50 \mathrm{~m} / \mathrm{z}$

$4200 \mathrm{~V}$

Scan End

$1500 \mathrm{~m} / \mathrm{z}$

Set Collision Cell RF

$-500 \mathrm{~V}$

$350.0 \mathrm{Vpp}$

nstrument

$\mathrm{UOH}-\mathrm{Chemistry}$

maXis

10138

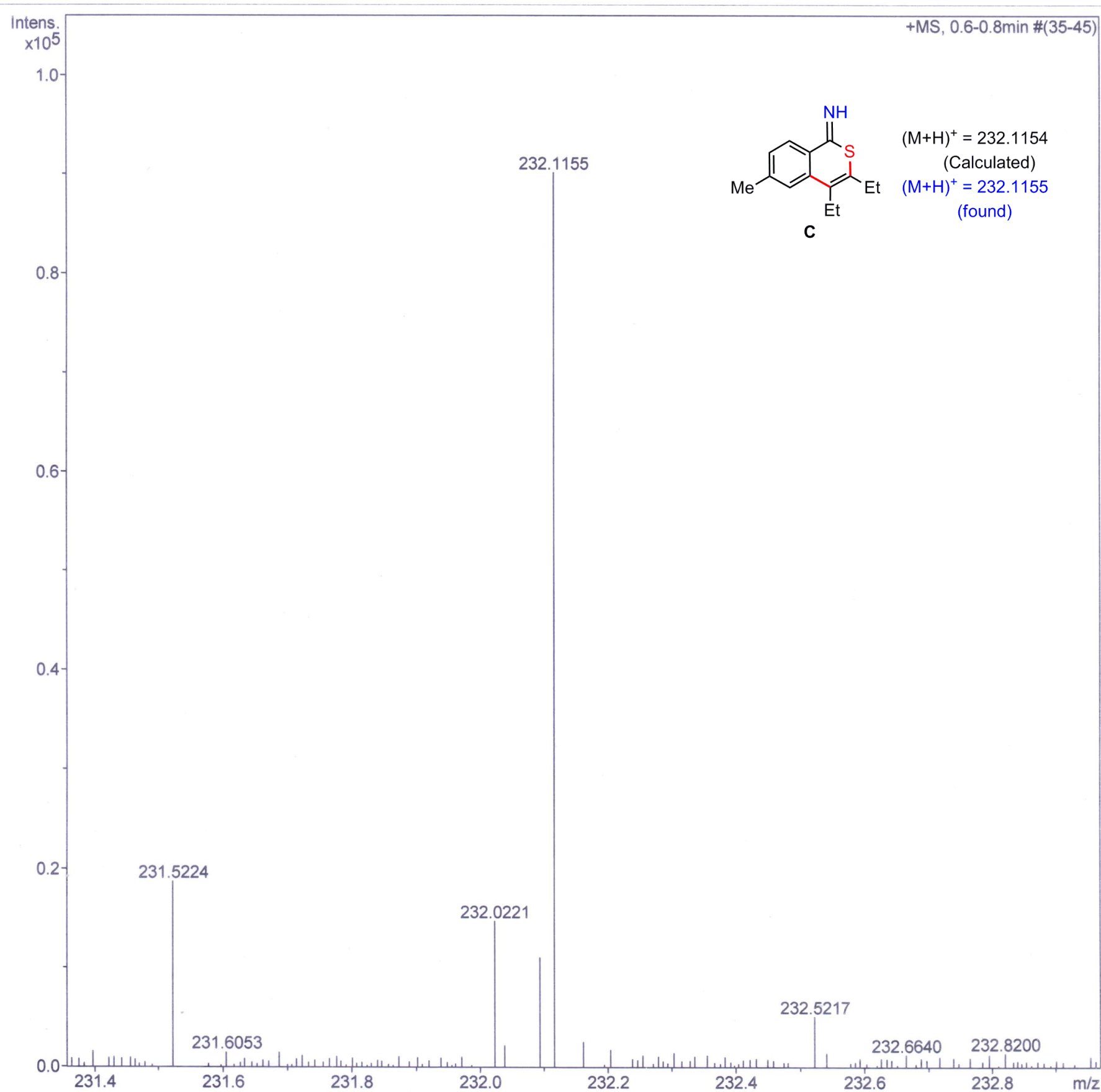

Bruker Compass DataAnalysis 4.0

printed:

12/10/2018 3:25:33 PM

Page 1 of 1 


\section{UOH -SCHOOL OF CHEMISTRY -HRMS}

\section{Analysis Info}

Analysis Name

Method

Sample Name

Comment
Acquisition Date

Operator

Instrument

2018Iprof aksIDECIMJ-138-15-2.

low Pos-R2.m

$\mathrm{MJ}-\overline{138}-\overline{15}$

$\mathrm{UOH}-\mathrm{Chemistry}$

maXis 10138

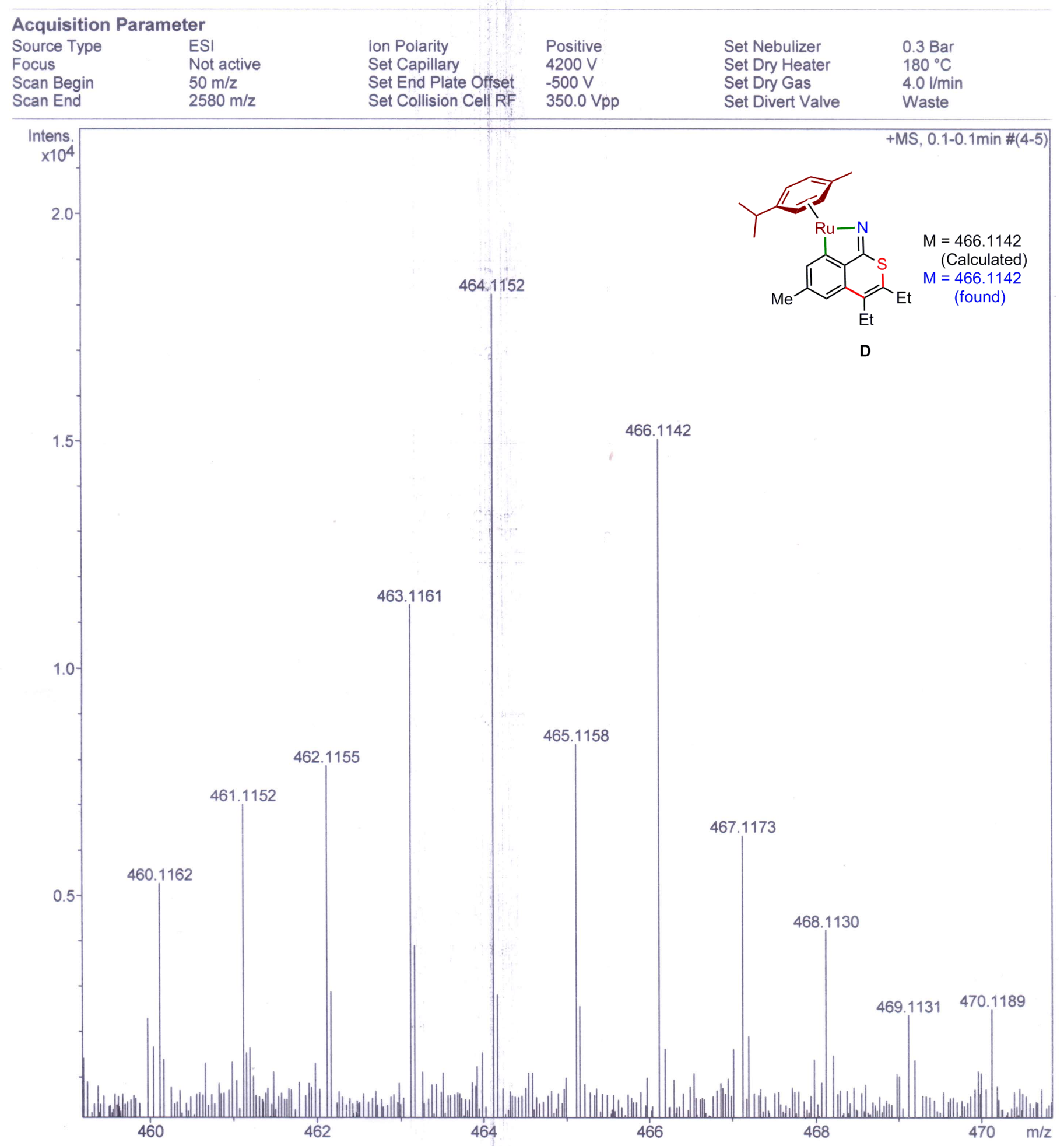




\section{UOH -SCHOOL OF CHEMISTRY -HRMS}

Analysis Info

Analysis Name

Method

Sample Name

D:IDatal2018Iprof aksIDECIMJ-138-15.d

tune low.m

MJ-138-15

Comment

\section{Acquisition Parameter}

Source Type

Scan Begin

ESI

$50 \mathrm{~m} / \mathrm{z}$

$1800 \mathrm{~m} / \mathrm{z}$

Set End Plate Offset $-500 \mathrm{~V}$

Set Collision Cell RF $\quad 350.0 \mathrm{Vpp}$

Acquisition Date

12/12/2018 4:04:45 PM

intens.

$\times 10^{5}$

2.5

$2.5-$

.

2.0

1.5

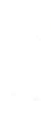

1.0

1.0

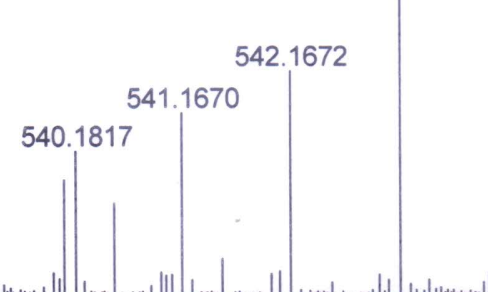

540

542
546.1828

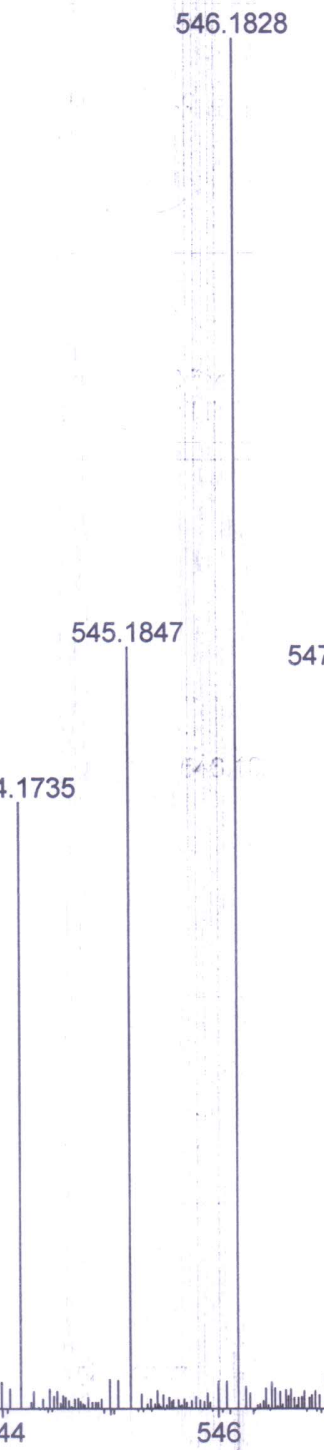

548.1925

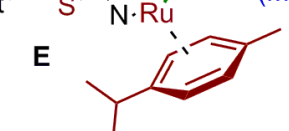

(Calculated)

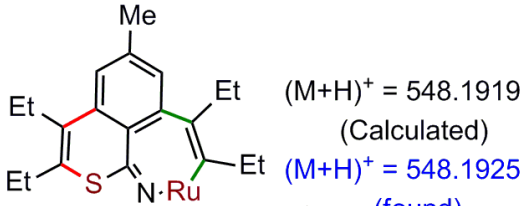

(found) 
MJ-61-15

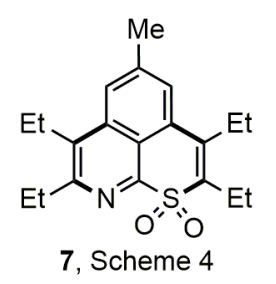

${ }^{1} \mathrm{H} \mathrm{NMR}\left(400 \mathrm{MHz}, \mathrm{CDCl}_{3}\right)$

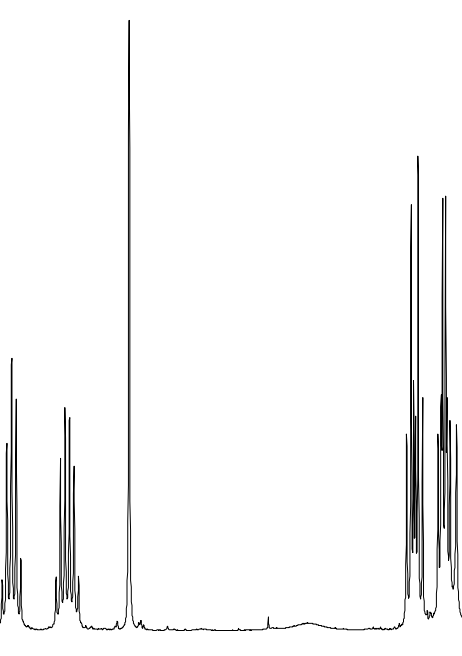

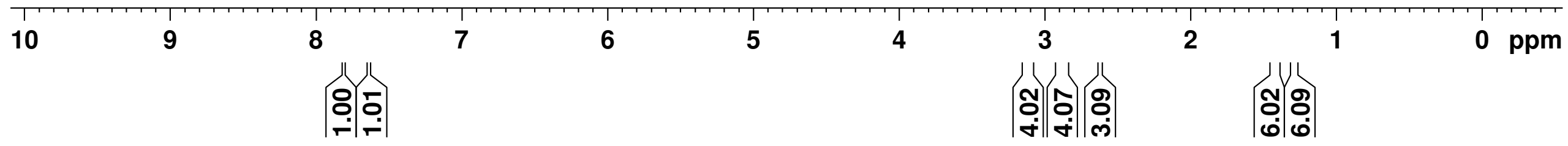




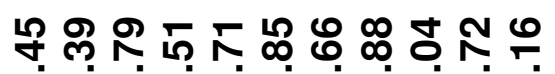

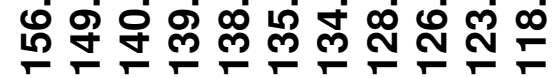

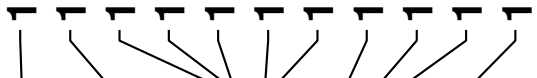

สำ

ヘト゚

$\checkmark$

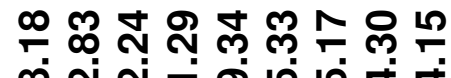

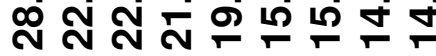

$\mathrm{MJ}-61-15$

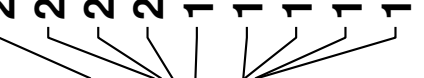

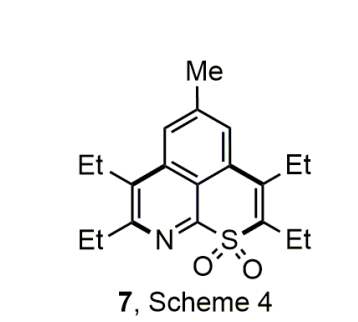

${ }^{13} \mathrm{C}\left\{{ }^{1} \mathrm{H}\right\}$ NMR $\left(126 \mathrm{MHz}, \mathrm{CDCl}_{3}\right)$

(3)

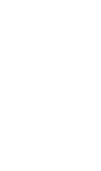

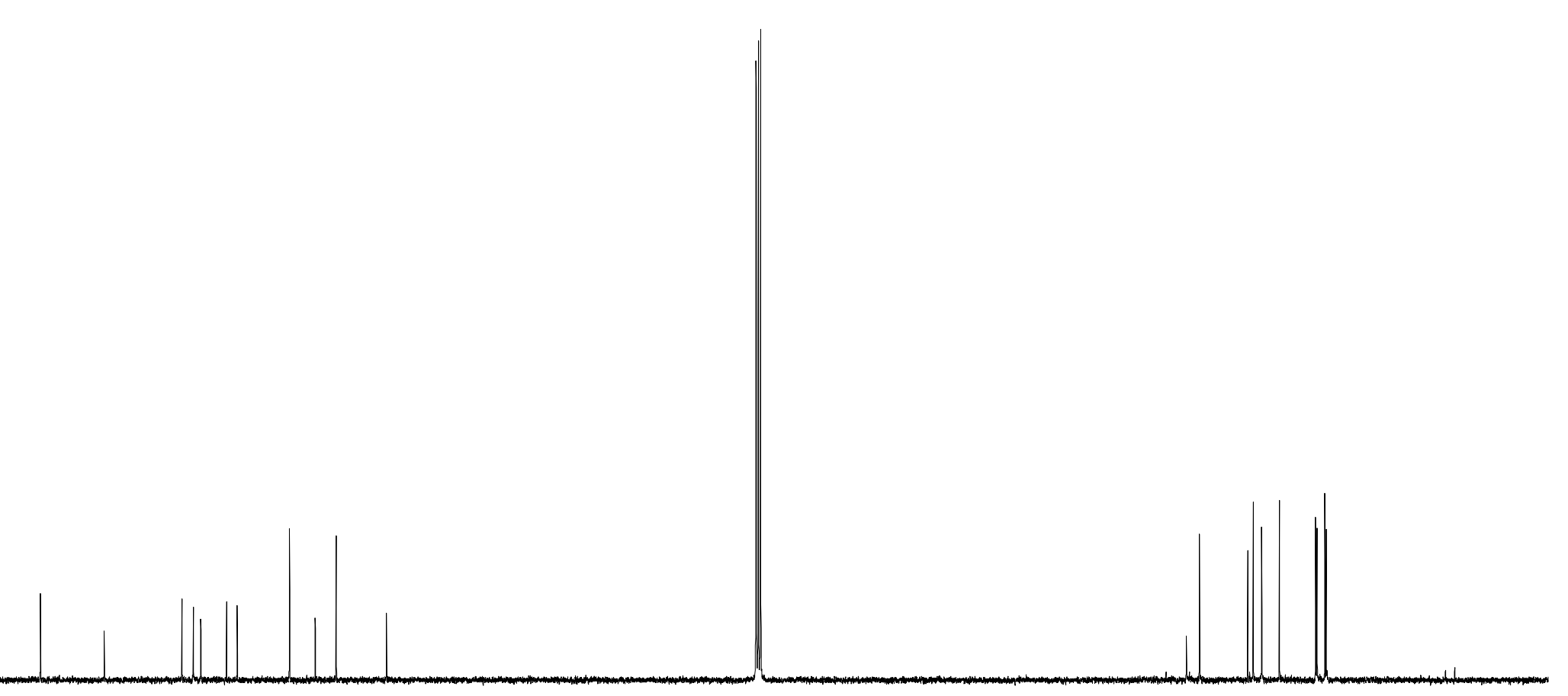

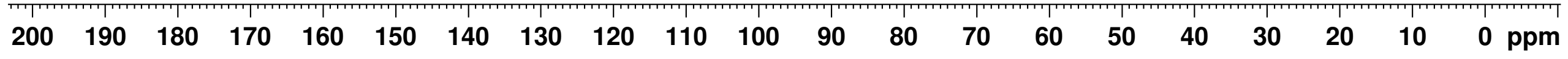


: §్ల

i

$\hat{1}$

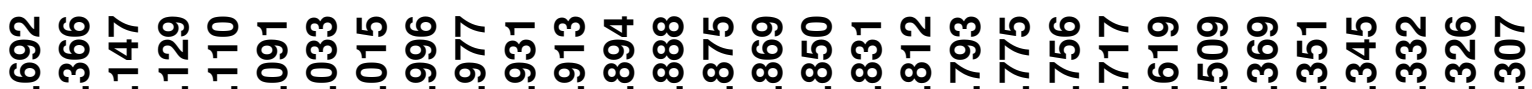

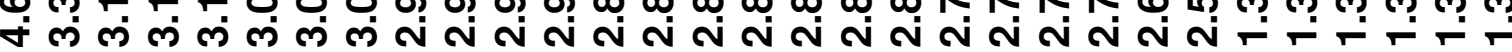

$\mathrm{MJ}-60-15$

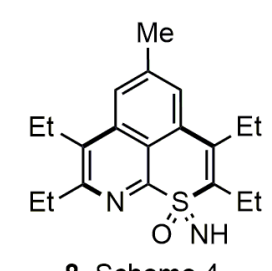

8, Scheme 4

${ }^{1} \mathrm{H}$ NMR (400 MHz, DMSO-d6)

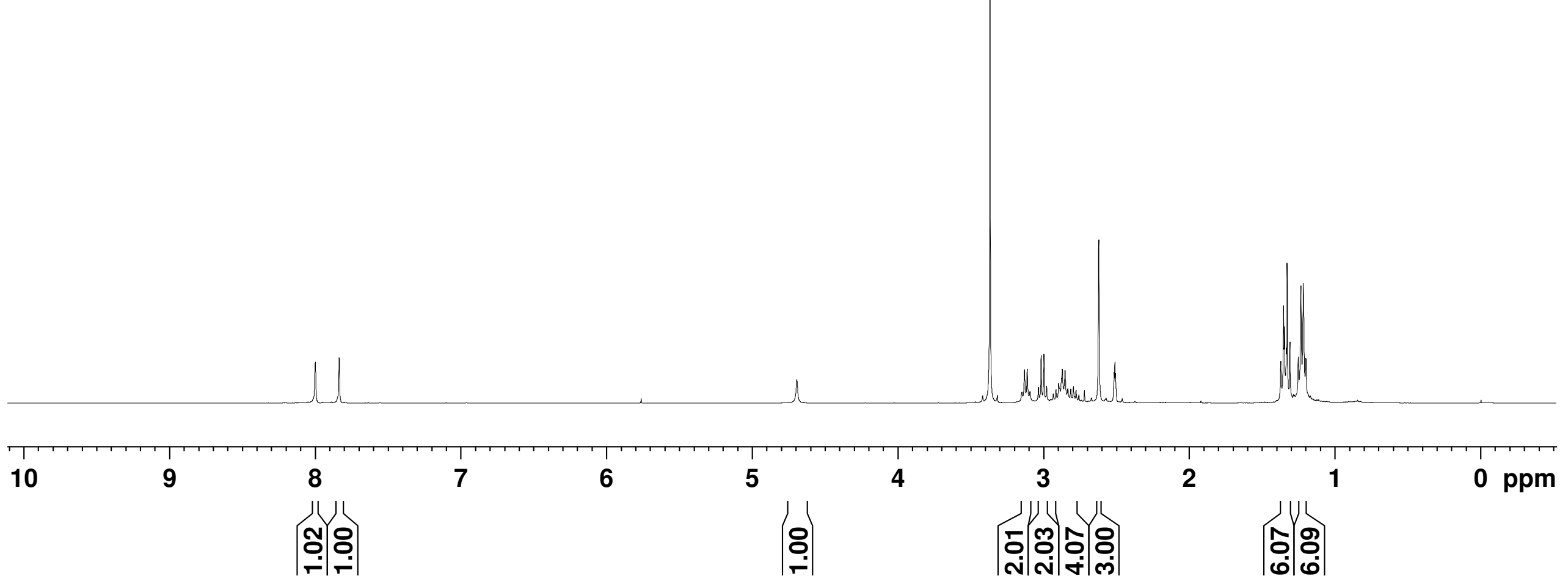




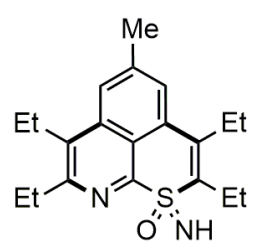

8, Scheme 4

${ }^{13} \mathrm{C}\left\{{ }^{1} \mathrm{H}\right\}$ NMR (101 MHz, DMSO-d6)

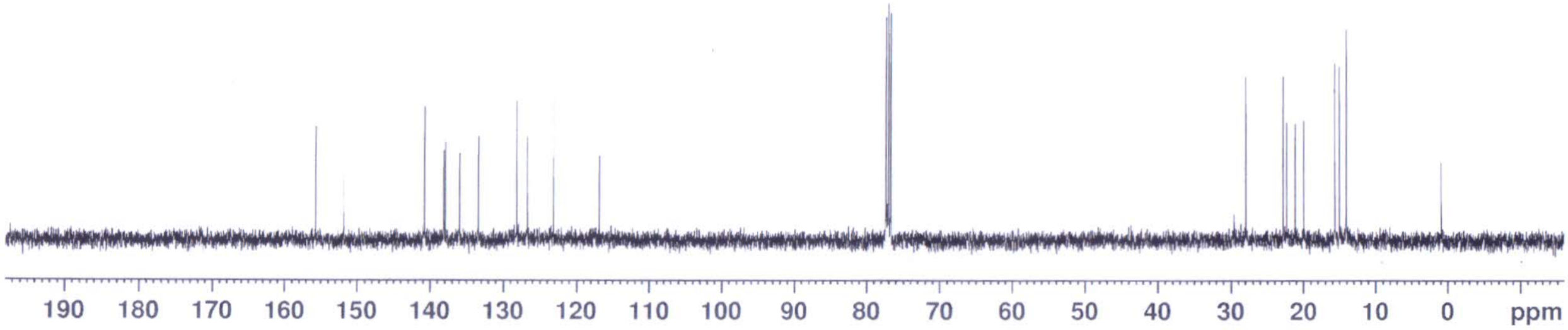


$\mathrm{MJ}-64-15$

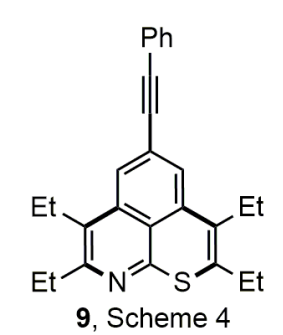

${ }^{1} \mathrm{H} \mathrm{NMR}\left(400 \mathrm{MHz}, \mathrm{CDCl}_{3}\right)$

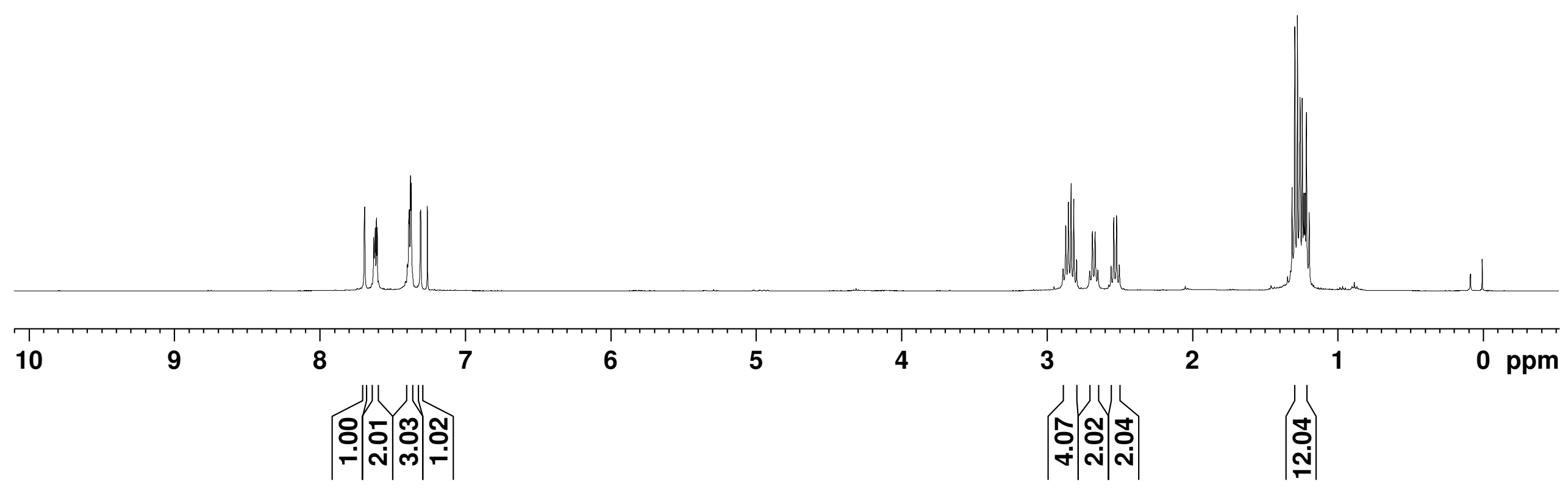



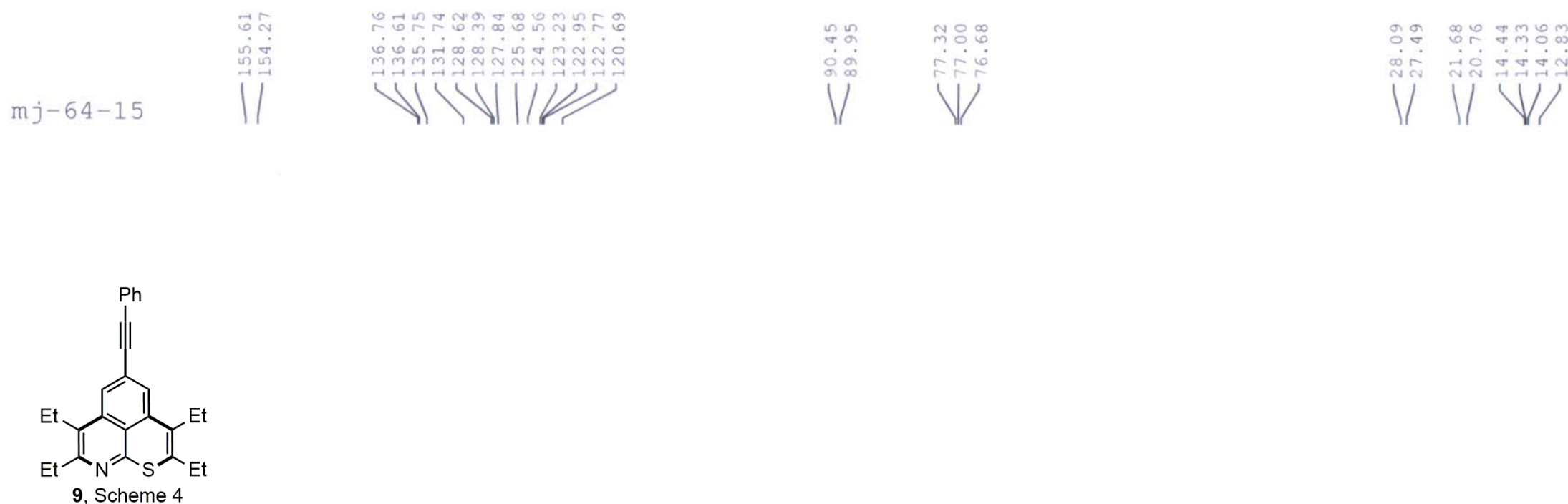

${ }^{13} \mathrm{C}\left\{{ }^{1} \mathrm{H}\right\}$ NMR $\left(101 \mathrm{MHz}, \mathrm{CDCl}_{3}\right)$

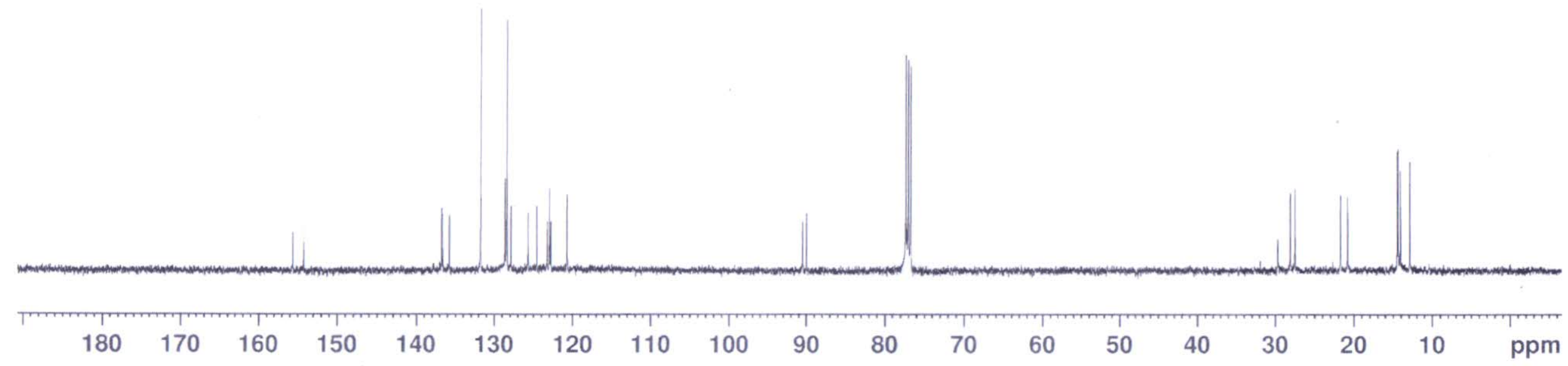


MJ-65-15

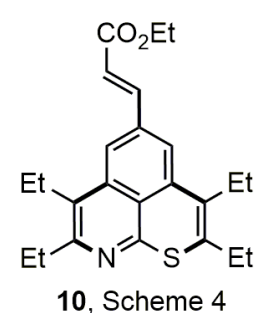

${ }^{1} \mathrm{H}$ NMR $\left(400 \mathrm{MHz}, \mathrm{CDCl}_{3}\right)$

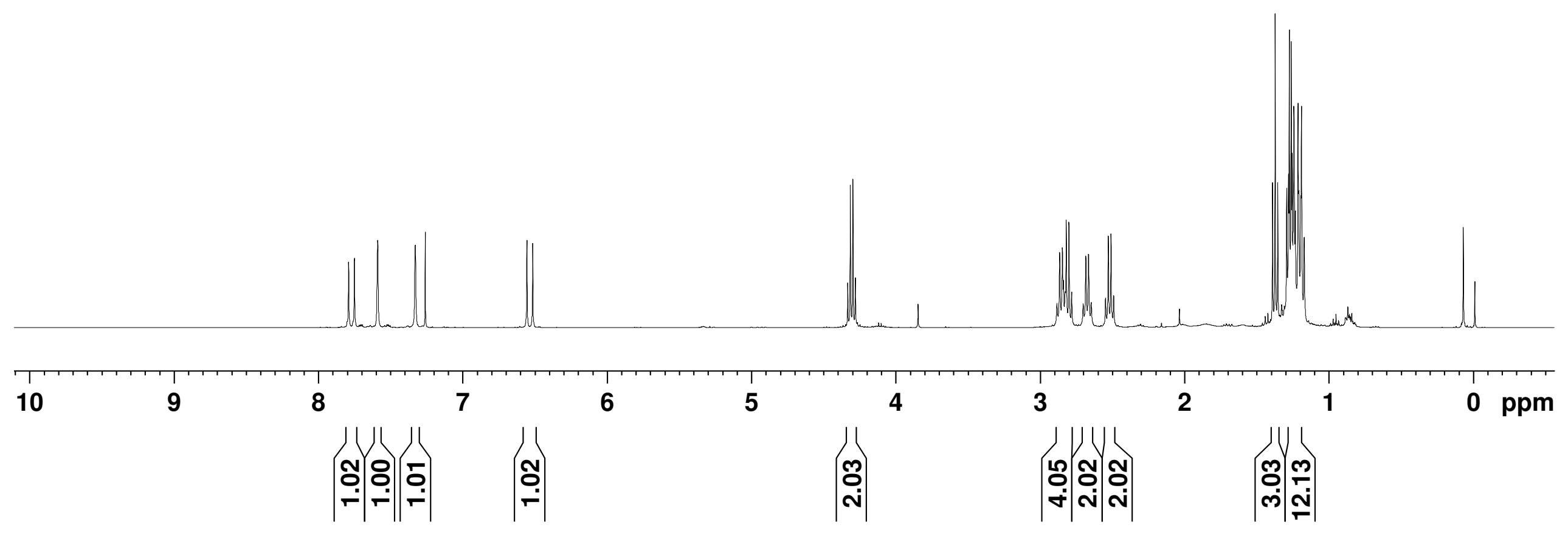


$m j-65-15$
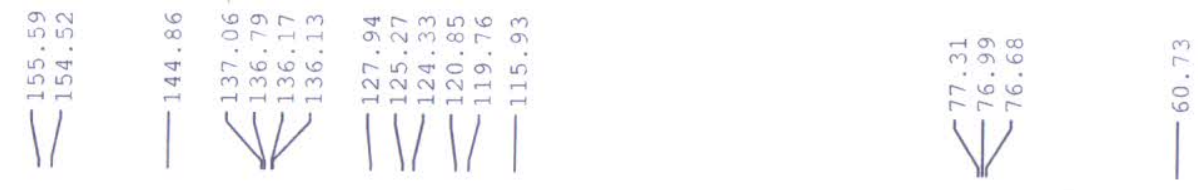

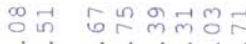

กัก ล่ง

$1 / V$

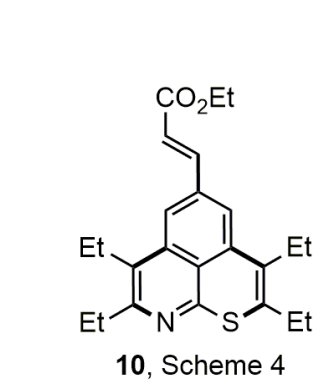

$\left.{ }^{13} \mathrm{C}^{1}{ }^{1} \mathrm{H}\right\}$ NMR $\left(101 \mathrm{MHz}, \mathrm{CDCl}_{3}\right)$

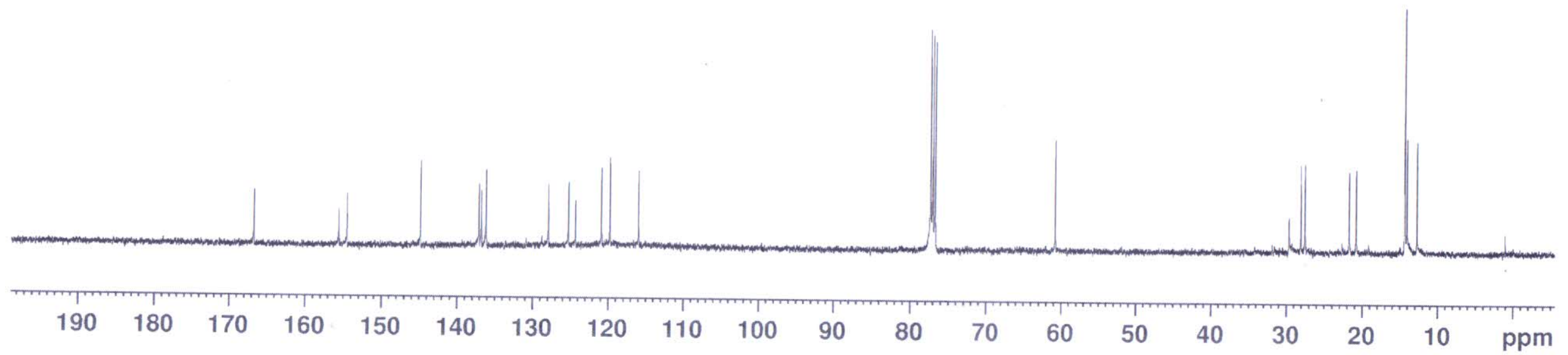




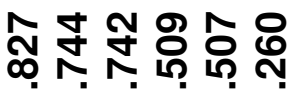

ヘNヘNヘ

M/

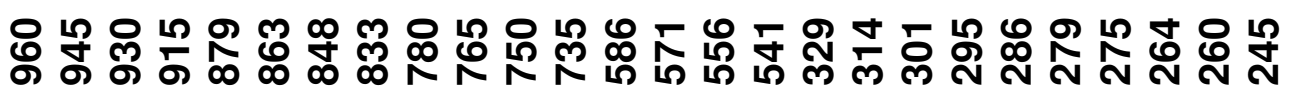

ง ง ง ง ง ง ง ง ง ง ง ง

$\mathrm{MJ}-63-15$

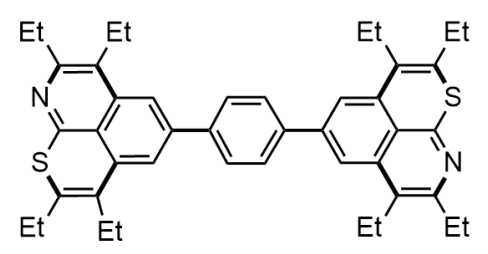

11, Scheme 4

${ }^{1} \mathrm{H} \mathrm{NMR}\left(500 \mathrm{MHz}, \mathrm{CDCl}_{3}\right)$

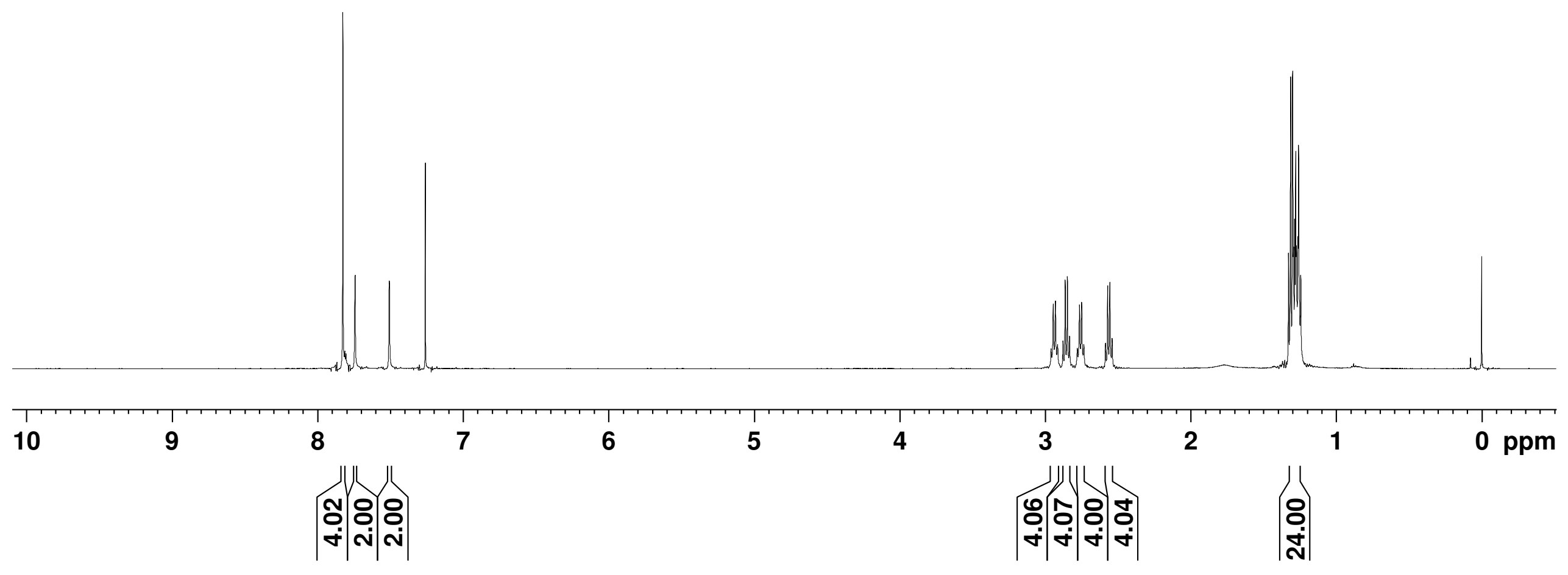




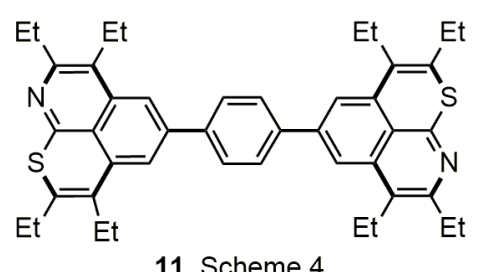

11, Scheme 4

${ }^{13} \mathrm{C}\left\{{ }^{1} \mathrm{H}\right\}$ NMR $\left(101 \mathrm{MHz}, \mathrm{CDCl}_{3}\right)$

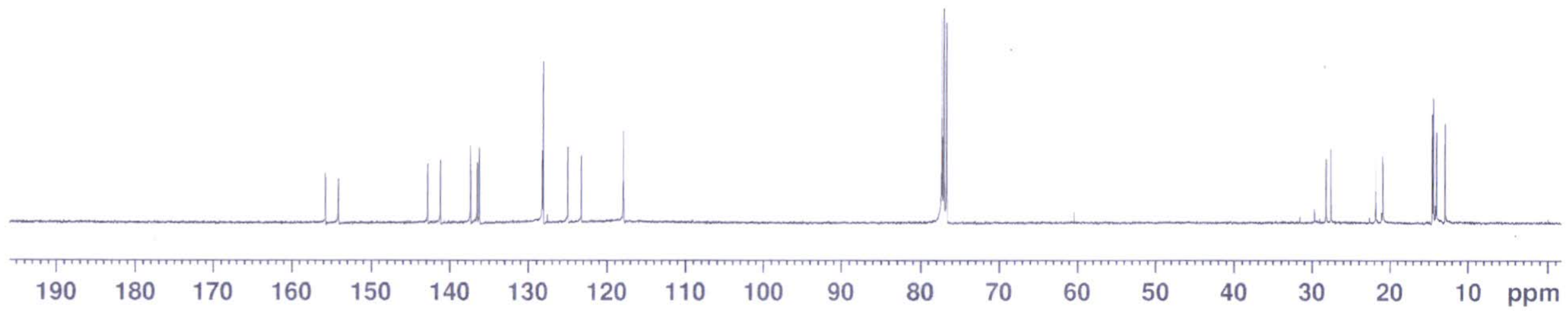

